\title{
A path model for geodesics in Euclidean buildings and its applications to representation theory
}

\author{
Michael Kapovich and John J. Millson
}

\begin{abstract}
In this paper we give a combinatorial characterization of projections of geodesics in Euclidean buildings to Weyl chambers. We apply these results to the representation theory of complex reductive Lie groups and to spherical Hecke rings associated with split nonarchimedean reductive Lie groups. Our main application is a generalization of the saturation theorem of Knutson and Tao for $\mathrm{SL}_{n}$ to other complex semisimple Lie groups.
\end{abstract}

Mathematics Subject Classification (2000). 20E42, 22E46.

Keywords. Euclidean buildings, LS path model, saturation theorem.

\section{Contents}

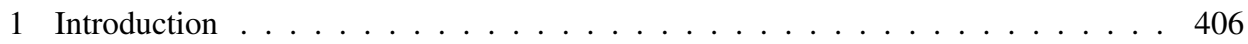

2 Definition and notation . . . . . . . . . . . . . . . . . . 413

2.1 Root systems and Coxeter complexes . . . . . . . . . . . . . . . . . . 413

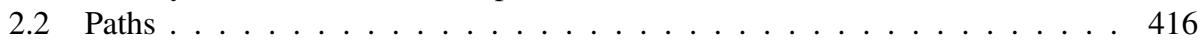

2.3 The saturation factors associated to a root system . . . . . . . . . . . 417

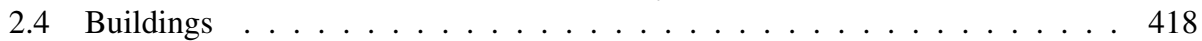

2.5 Generalized distances and lengths in buildings . . . . . . . . . . . . . . . 421

2.6 The Hecke ring . . . . . . . . . . . . . . . . . . . . . . . . . . . . . . . . . . . . . . 422

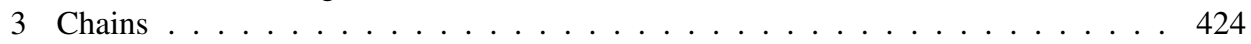

3.1 Absolute chains . . . . . . . . . . . . . . . . . . . . 424

3.2 Relative chains . . . . . . . . . . . . . . . . . . . . 429

3.3 Hecke paths . . . . . . . . . . . . . . . . . . . 432

3.4 A compactness theorem . . . . . . . . . . . . . . . . . . . . . . . . . . . . . . . . . . . . . . . . . . .

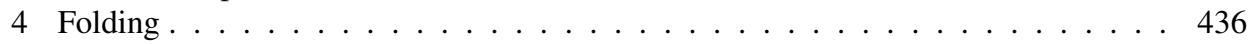

4.1 Folding via retraction . . . . . . . . . . . . . . . . 436

4.2 Converting folded triangles in spherical buildings into chains . . . . . . . . . 441

4.3 Folding polygons in Euclidean buildings . . . . . . . . . . . . . . . . . 446

5 Littelmann polygons . . . . . . . . . . . . . . . . . . . 452

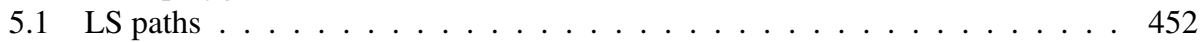

5.2 Root operators . . . . . . . . . . . . . . . . 456 
5.3 Generalized LS paths . . . . . . . . . . . . . . . . . . . 459

5.4 Path model for the representation theory of Lie groups . . . . . . . . . . . . . 464

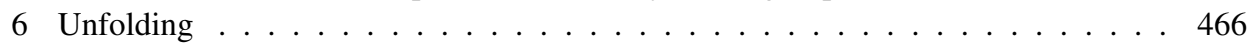

6.1 Unfolding Littelmann triangles . . . . . . . . . . . . . . . . . . . . . . . . . . . . . . 466

6.2 Characterization of folded triangles . . . . . . . . . . . . . . . . 470

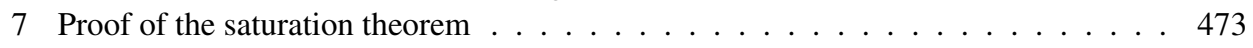

References . . . . . . . . . . . . . . . . . . . 478

\section{Introduction}

Let $\underline{G}$ be a $\mathbb{Q}$-split reductive algebraic group defined over $\mathbb{Z}$ and $\underline{G}^{\vee}$ be its Langlands' dual. In this paper we continue our study (which we began in [KLM3]) of the interaction between the representation theory of the group $G^{\vee}:=\underline{G}^{\vee}(\mathbb{C})$ and geometry of the Bruhat-Tits building associated with the nonarchimedean group $G=\underline{G}(\mathbb{K})$, where $\mathbb{K}$ is a complete field with discrete valuation. We restrict ourselves to the case when $\mathbb{K}$ is a local field, in which case, algebraically speaking, we will be studying the relation between the representation ring of the group $G^{\vee}$ and the spherical Hecke algebra $\mathscr{H}_{G}$ associated with $G$.

In his papers [L1], [L2], P. Littelmann introduced a path model for the representations of complex reductive Lie groups $G^{\vee}$. The Littelmann path model gives a method to compute the structure constants of the representation ring of $G^{\vee}$ by counting certain piecewise linear paths, called LS paths.

In this paper we define a class of piecewise-linear paths in $\Delta$, a Weyl chamber of the Weyl group $W$ of $G^{\vee}$. These paths will be called Hecke paths (see Definition 3.27) because of their connection with $\mathscr{H}_{G}$. We will prove that a path $p$ in $\Delta$ is a Hecke path if and only if $p$ is the projection into $\Delta$ of a geodesic segment in the Euclidean (Bruhat-Tits) building $X$ associated with $G$. Thus, unlike LS paths which had to be invented, the Hecke paths appear very naturally as projections of geodesic segments. Hecke paths are defined by eliminating one of the axioms for LS paths, therefore each $L S$ path for $G^{\vee}$ is a Hecke path for $G$.

The converse relation is more subtle and is discussed later in the introduction. To state our main results we need a definition of $k_{R}$, the saturation factor of the root system $R$ of the group $\underline{G}$. Let $\alpha_{1}, \ldots, \alpha_{l} \in R$ be the simple roots (corresponding to $\Delta$ ). Let $\theta$ be the highest root and define positive integers $m_{1}, \ldots, m_{l}$ by

$$
\theta=\sum_{i=1}^{l} m_{i} \alpha_{i} .
$$

Then $k_{R}$ is the least common multiple of the numbers $m_{i}, i=1, \ldots, l$. We refer to Section 2.3 for the computation of $k_{R}$. 
Below, $L$ is the character lattice of a maximal torus in $G^{\vee}$ (so that $\Delta \subset L \otimes \mathbb{R}$ ), $Q\left(R^{\vee}\right)$ is the root lattice of $R^{\vee}$. Our main result is the following theorem (see Section 7), which, in a weaker form, has been conjectured by S. Kumar:

Theorem 1.1 (Saturation theorem). Let $G^{\vee}$ and $L$ be as above. Suppose that $\alpha, \beta, \gamma \in$ $L$ are dominant characters such that $\alpha+\beta+\gamma \in Q\left(R^{\vee}\right)$ and that there exists $N \in \mathbb{N}$ so that

$$
\left(V_{N \alpha} \otimes V_{N \beta} \otimes V_{N \gamma}\right)^{G^{\vee}} \neq 0 .
$$

Then for $k=k_{R}^{2}$ we have:

$$
\left(V_{k \alpha} \otimes V_{k \beta} \otimes V_{k \gamma}\right)^{G^{\vee}} \neq 0 .
$$

Here and in what follows $V_{\lambda}$ is the irreducible representation of $G^{\vee}$ associated with the dominant weight $\lambda$ of $G^{\vee}$. Also it will be convenient to introduce integers $n_{\alpha, \beta}\left(\gamma^{*}\right)$, the structure constants of the representation ring of the group $G^{\vee}$. (Here and in what follows, $\gamma^{*}$ is the dominant weight contragredient to $\gamma$.) Hence $n_{\alpha, \beta}\left(\gamma^{*}\right)$ are defined by the equation

$$
V_{\alpha} \otimes V_{\beta}=\bigoplus_{\gamma} n_{\alpha, \beta}\left(\gamma^{*}\right) V_{\gamma^{*}}
$$

Here the right-hand side is the decomposition of the tensor product of the irreducible representations $V_{\alpha}$ and $V_{\beta}$ into a direct sum of irreducible representations. We can then formulate the above theorem as

$$
n_{N \alpha, N \beta}\left(N \gamma^{*}\right) \neq 0 \Longrightarrow n_{k \alpha, k \beta}\left(k \gamma^{*}\right) \neq 0 .
$$

We will freely move back and forth between the symmetric formulation of saturation in Theorem 1.1 and the asymmetric formulation immediately above.

As an immediate corollary of Theorem 1.1 we obtain a new proof of the saturation theorem of A. Knutson and T. Tao [KT]:

Corollary 1.2. Suppose that $R=A_{l}$, i.e., the semisimple part of $G^{\vee}$ is locally isomorphic to $\mathrm{SL}_{l+1}$. Suppose further that $\alpha, \beta, \gamma$ are dominant characters such that $\alpha+\beta+\gamma \in Q\left(R^{\vee}\right)$ and that there exists $N \in \mathbb{N}$ so that

$$
\left(V_{N \alpha} \otimes V_{N \beta} \otimes V_{N \gamma}\right)^{G^{\vee}} \neq 0 .
$$

Then

$$
\left(V_{\alpha} \otimes V_{\beta} \otimes V_{\gamma}\right)^{G^{\vee}} \neq 0 .
$$

Another proof of this theorem was given by H. Derksen and J. Weyman in [DW]. However the proofs of [KT] and [DW] do not work for root systems different from $A_{l}$. 
Question 1.3. Is it true that if $G$ is a simple simply-laced group, then in Theorem1.1 one can always take $k=1$ and in the case of non-simply laced groups the smallest $k$ which suffices is $k=2$ ?

The affirmative answer to this question is supported by the odd orthogonal groups, symplectic groups and $G_{2}$, when one gets the saturation constant $k=2$ rather than $2^{2}$ and $6^{2}$ [KLM3], [BK], the group $\operatorname{Spin}(8)$, when the saturation constant equals 1 $[\mathrm{KKM}]$, as well as by a number of computer experiments with the exceptional root systems and the root systems $D_{l}$.

Using the results of [KLM2], [KLM3] one can reformulate Theorem 1.1 as follows:

Theorem 1.4. There exists a convex homogeneous cone $D_{3} \subset \Delta^{3}$, defined by the generalized triangle inequalities, which depends only on the Weyl group W, so that the following hold:

(1) If a triple $(\alpha, \beta, \gamma) \in(\Delta \cap L)^{3}$ satisfies

$$
\left(V_{\alpha} \otimes V_{\beta} \otimes V_{\gamma}\right)^{G^{\vee}} \neq 0,
$$

then $(\alpha, \beta, \gamma) \in D_{3}$ and

$$
\alpha+\beta+\gamma \in Q\left(R^{\vee}\right) .
$$

(2) "Conversely", if $(\alpha, \beta, \gamma) \in k_{R}^{2} \cdot L^{3} \cap D_{3}$ and $\alpha+\beta+\gamma \in k_{R}^{2} \cdot Q\left(R^{\vee}\right)$, then

$$
\left(V_{\alpha} \otimes V_{\beta} \otimes V_{\gamma}\right)^{G^{\vee}} \neq 0 .
$$

We now outline the steps required to prove the Theorem 1.1. We will need several facts about Hecke rings $\mathscr{H}$. Let $\mathcal{O}$ denote the valuation ring of $\mathbb{K}$, then $K:=\underline{G}(\mathcal{O})$ is a maximal compact subgroup in $G$. The lattice $L$ defined above is the cocharacter lattice of a maximal torus $T \subset G$. The Hecke ring $\mathscr{H}$, as a $\mathbb{Z}$-module, is freely generated by the characteristic functions $\left\{c_{\lambda}: \lambda \in L \cap \Delta\right\}$. The multiplication on $\mathscr{H}$ is defined via the convolution product $\star$. Then the structure constants $m_{\alpha, \beta}(\gamma)$ of $\mathscr{H}$ are defined by

$$
c_{\alpha} \star c_{\beta}=\sum_{\gamma} m_{\alpha, \beta}(\gamma) c_{\gamma}
$$

We refer the reader to [Gro], [KLM3] and to Section 2.6 of this paper for more details.

Let $o \in X$ be the special vertex of the building $X$ which is fixed by $K$. In Section 2.5 we define the notion $d_{\Delta}(x, y)$ of the $\Delta$-valued distance between points $x, y \in X$. Given a piecewise-geodesic path $p$ in $X$ we define its $\Delta$-length as the sum of $\Delta$-distances between the consecutive vertices. 
The structure constants for $\mathscr{H}$ are related to the geometry of $X$ via the following

Theorem 1.5 ([KLM3], Theorem 8.12). The number $m_{\alpha, \beta}(\gamma)$ is equal to the product of a certain positive constant by the number of geodesic triangles $T \subset X$ whose vertices are special vertices of $X$ with the first vertex equal to o and whose $\Delta$-side lengths are $\alpha, \beta, \gamma^{*}$.

Theorem 1.1 is essentially Statement 3, which follows from Statements 1 and 2, of the following

Theorem 1.6. Set $\ell:=k_{R}$.

(1) Suppose that $(\alpha, \beta, \gamma) \in D_{3} \cap L^{3}$ and $\alpha+\beta+\gamma \in Q\left(R^{\vee}\right)$. Then the structure constants $m \cdot, \cdot(\cdot)$ of the Hecke ring of the group $G$ satisfy

$$
m_{\ell \alpha, \ell \beta}(\ell \gamma) \neq 0 \text {. }
$$

(2) Suppose that $\alpha, \beta, \gamma$ are dominant coweights of $\underline{G}$ such that $m_{\alpha, \beta}(\gamma) \neq 0$. Then

$$
n_{\ell \alpha, \ell \beta}(\ell \gamma) \neq 0
$$

(3) As a consequence of (1) and (2) we have: Suppose that $(\alpha, \beta, \gamma) \in D_{3} \cap L^{3}$ and $\alpha+\beta+\gamma \in Q\left(R^{\vee}\right)$. Then

$$
n_{\ell^{2} \alpha, \ell^{2} \beta}\left(\ell^{2} \gamma\right) \neq 0
$$

Remark 1.7. (a) Part 1 of the above theorem was proven in [KLM3]. Thus the point of this paper is to prove Part 2 of the above theorem.

(b) Examples in [KLM3] show that both implications

$$
(\alpha, \beta, \gamma) \in D_{3} \cap L^{3}, \alpha+\beta+\gamma \in Q\left(R^{\vee}\right) \Longrightarrow m_{\alpha, \beta}(\gamma) \neq 0
$$

and

$$
m_{\alpha, \beta}(\gamma) \neq 0 \Longrightarrow n_{\alpha, \beta}(\gamma) \neq 0
$$

are false for the groups $G_{2}$ and $\mathrm{SO}(5)$. Therefore the dilation by $k_{R}$ in both cases is necessary at least for these groups.

More generally, we prove (Theorem 7.4, p. 475):

Theorem 1.8. Suppose that $\alpha, \beta, \gamma \in L$ are dominant weights so that one of them is the sum of minuscule weights. Then

$$
m_{\alpha, \beta}(\gamma) \neq 0 \Longrightarrow n_{\alpha, \beta}(\gamma) \neq 0 \text {. }
$$


The proof of Part 2 of Theorem 1.6 proceeds as follows. In Section 6.2 we prove a characterization theorem for folded triangles which implies:

Theorem 1.9. There exists a geodesic triangle $T \subset X$ whose vertices are special vertices of $X$ and whose $\Delta$-side lengths are $\alpha, \beta, \gamma^{*}$ if and only if there exists a Hecke path $p:[0,1] \rightarrow \Delta$ of $\Delta$-length $\beta$ so that

$$
p(0)=\alpha, \quad p(1)=\gamma .
$$

The directed segments $\pi_{\alpha}$, the Hecke path $p$ and the (reversed) directed segment $\pi_{\gamma}$ fit together to form a "broken triangle", see Figure 1. Here and in what follows $\pi_{\lambda}$ is the geodesic path parameterizing the directed segment $\overrightarrow{o x}=\lambda$.

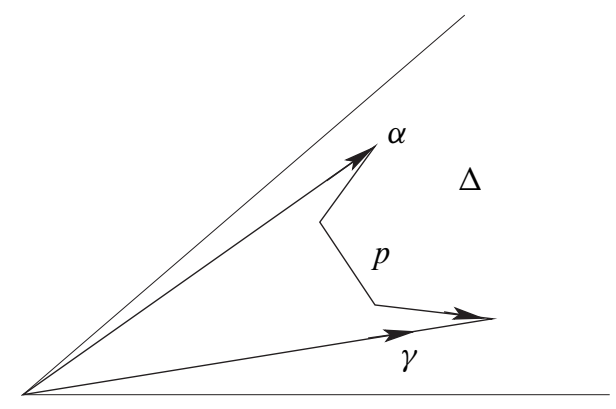

Figure 1. A broken triangle.

Then, by combining theorems 1.5 and 1.9 , we obtain

Theorem 1.10. $m_{\alpha, \beta}(\gamma) \neq 0$ if and only if there exists a Hecke path $p:[0,1] \rightarrow \Delta$ of $\Delta$-length $\beta$ so that

$$
p(0)=\alpha, \quad p(1)=\gamma .
$$

This statement is an analogue of Littelmann's theorem which relates structure constants $n_{\alpha, \beta}(\gamma)$ of the representation ring with LS paths. The problem however is that not every Hecke path is an LS path (even for the group SL(3)).

In order to prove Theorem 1.1 our "path model" for the nonvanishing of the Hecke structure constants must be generalized to a model where Hecke paths are replaced by generalized Hecke paths (we also have to replace the LS-paths by generalized LS paths). More precisely, begin with a geodesic triangle $[x, y, z] \subset X$ whose vertices are special vertices of $X$ and whose $\Delta$-side lengths are $\beta, \gamma^{*}, \alpha$. Now replace the geodesic segment $\overline{x y}$ with a certain piecewise-geodesic path $\tilde{p} \subset X$ connecting $x$ and $y$ in $X$, and which is contained in the 1-skeleton of a single apartment in $X$. We then show that the projection $p$ of $\tilde{p}$ to $\Delta$ is a generalized Hecke path. The $\Delta$-length of the path $p$ still equals $d_{\Delta}(x, y)=\beta$. 
The generalized Hecke paths have the advantage over Hecke paths that their break points occur only at vertices of the building. By using this observation we obtain

Theorem 1.11. If $p$ is a generalized Hecke path with $p(0)=0$, then $k_{R} \cdot p$. The image of $p$ under dilation by $k_{R}$ is a generalized LS path of $\Delta$-length equal to $k_{R}$ length ${ }_{\Delta}(p)$.

Lastly, for generalized LS paths we prove, by modifying slightly Littelmann's arguments, the following:

Theorem 1.12. $n_{\alpha, \beta}(\gamma) \neq 0$ if and only if there exists a generalized LS path $q$ with $\Delta$-length $\beta$ so that

$$
\alpha+q(1)=\gamma
$$

and the concatenation $\pi_{\alpha} * q$ is contained in $\Delta$.

Theorem 1.1 is now obtained in 2 steps, the first of which is contained in [KLM3] and the second is at the heart of the present paper:

Step 1. It was shown in [KLM3], Theorem 9.17, that

$$
n_{N \alpha, N \beta}(N \gamma) \neq 0 \Longrightarrow m_{N \alpha, N \beta}(N \gamma) \neq 0
$$

Therefore, the vector ( $\left.N \alpha, N \beta, N \gamma^{*}\right)$ belongs to the homogeneous cone $D_{3}$. Hence, by Part 1 of Theorem 1.6 we conclude that $m_{\ell \alpha, \ell \beta}(\ell \gamma) \neq 0$.

Step 2. $m_{\ell \alpha, \ell \beta}(\ell \gamma) \neq 0$ implies existence of a geodesic triangle $[z, x, y] \subset X$ with the special vertices and $\Delta$-side lengths $\ell \alpha, \ell \beta, \ell \gamma^{*}$. Then by projecting the corresponding path $\tilde{p}$ to $\Delta$ and dilating it by $k_{R}$ we obtain a path $k \alpha+q(t)$ in $\Delta$ connecting $k \alpha$ to $k \gamma^{*}$, where $q(t)$ is a generalized LS path of $\Delta$-length $k \beta$. (Recall that $k=\ell^{2}$.) Therefore, by appealing to Theorem 1.12 , we see that

$$
n_{k \alpha, k \beta}(k \gamma) \neq 0
$$

which concludes the proof.

This paper is organized as follows. Preliminary material is discussed in Section 2 , where we review the concepts of Coxeter complexes, buildings and piecewise-linear paths in buildings, as well as the generalized distances in buildings. In Section 3 we define the notion of chains, which is essentially due to Littelmann. This concept allows one to define both LS paths and Hecke paths, as well as to relate Hecke paths and foldings of geodesic paths, which is discussed in the next section.

The main technical tool of this paper is the concept of folding of geodesics in a building into an apartment, which is done via retraction of the entire building to an apartment or chamber. ${ }^{1}$ Properties of foldings are discussed in Section 4. In

\footnotetext{
${ }^{1} \mathrm{~A}$ similar idea is used by S. Gaussent and P. Littelmann in [GL], where they fold galleries in a building to galleries in an apartment.
} 
Section 4.3 we prove that the image of each geodesic segment in $X$ under folding $f: X \rightarrow \Delta$ of $X$ to a Weyl chamber, is a Hecke path, Theorem 4.16. We then prove a partial converse to this result, i.e., that each Hecke path which satisfies the simple chain condition can be unfolded in $X$. We also find a necessary and sufficient condition for unfolding of a path $p$ which is local, i.e., it depends only on germs of the path $p$ at its break points.

In Section 5 we review Littelmann's path model for the representation theory of complex semisimple Lie groups, in particular we discuss LS paths and generalized LS paths as well as raising and lowering operators.

We use approximation of LS paths by paths satisfying the simple chain condition to unfold LS paths in $X$, Theorem 6.1 in Section 6.1. Although there are Hecke paths which are not LS paths, since the unfolding condition is local, by restricting the root system we reduce the general unfolding problem to the case of the LS paths. We thus establish that a path in $\Delta$ is unfoldable in $X$ it and only if it is a Hecke path, this is done in Section 6.2, Theorem 6.5. The reader interested only in the proof of the saturation theorem can omit this section.

We prove the saturation theorem in Section 7, Corollary 7.3. As we stated above, the idea of the proof is to replace Hecke paths with piecewise-linear paths contained in the 1-skeleton of the Euclidean Coxeter complex. We show that dilation by $k_{R}$ of such a path results in a generalized LS path which in turn suffices for finding nonzero invariant vectors in triple tensor products.

Hecke paths and folded galleries. It is interesting to ask what the relationship is between our paper using Hecke paths and the results of S. Gaussent and P. Littelmann in [GL] and others using positively folded galleries:

(a) Hecke paths correspond to the positively folded galleries defined in [GL]. However this correspondence can be established only after the folding-unfolding results of the present paper are proven. Therefore it appears that one cannot prove our results characterizing Hecke paths as projections of geodesic segments in building using the results of [GL] and vice versa.

(b) In [Sc], C. Schwer has used the gallery approach to compute the Hecke structure constants $m_{\alpha, \beta}(\gamma)$. In an earlier version of this paper we applied our theory to compute some Hecke structure coefficients. Thus both Hecke path and gallery models can be used to compute the structure constants of the spherical Hecke algebra.

(c) One can prove that

$$
n_{\alpha, \beta}(\gamma) \neq 0 \Longrightarrow m_{\alpha, \beta}(\gamma) \neq 0
$$

[KLM3], Theorem 9.17, using both Hecke paths (as it is done in Section 6.2) and positively folded galleries.

(d) There does not seem to be a direct way to carry over our proof of the main Theorem 1.1 or part 2 of Theorem 1.6 to a proof using positively folded galleries. 
It is clear from the above, that one of the critical steps in our argument is provided by Theorem 1.11 stating that dilation by $k_{R}$ converts generalized Hecke paths to generalized LS paths. But there does not appear to be a way to "stretch" a folded gallery by the factor $k_{R}$-or, more generally, to produce an LS gallery from a positively folded gallery. This is an advantage of the Hecke paths, which are geometric objects, over positively folded galleries, which are combinatorial objects. Even when $k_{R}=1$, there is no obvious (at least to us) reason why existence of a positively folded gallery would imply existence of an LS gallery. We note that if $k_{R} \neq 1$ then the existence of a positively folded gallery does not imply the existence of an LS gallery, there are counterexamples for $\mathrm{SO}(5)$ and $G_{2}$.

Acknowledgments. This paper grew out of our joint work [KLM1], [KLM2], [KLM3] with Bernhard Leeb. We are grateful to him for all his ideas that he contributed to those papers, especially the idea of folding that came out of his contribution to [KLM3]. We are also grateful to Tom Haines for valuable discussions. We thank the referee for his/her remarks. Part of the work on this paper was done when the first author was visiting the Max Planck Institute (Bonn), during this work he was also supported by the NSF grant DMS-02-03045 and DMS-04-05180. The second author was supported by the NSF grant DMS-01-04006 and DMS-04-05606. Together, the authors were supported by the NSF grant DMS-05-54349. The authors gratefully acknowledge support of these institutions.

\section{Definition and notation}

2.1. Root systems and Coxeter complexes. A (discrete, nonnegatively curved) Coxeter complex is a pair $(A, W)$, where $A$ is either a Euclidean space or the unit sphere and $W$ is a discrete reflection group acting on $A$. The rank of the Coxeter complex is the dimension of $A$. The group $W$ is called Weyl group of the Coxeter complex. It is called an affine Weyl group if $A$ is a Euclidean space.

An isomorphism of Coxeter complexes $(A, W),\left(A^{\prime}, W^{\prime}\right)$ is an isometry $\iota: A \rightarrow A^{\prime}$ so that

$$
\iota W \iota^{-1}=W^{\prime}
$$

Walls in $(A, W)$ are fixed point sets of reflections $\tau \in W$. A point $x \in A$ is called regular if it does not belong to any wall and singular otherwise. The closure of each connected component of the set of regular points is called an alcove in the Euclidean case and a chamber in the spherical case.

In the case when $W$ acts cocompactly on $A$, each alcove (resp. chamber) is a product (resp. join) of simplices and $(A, W)$ determines structure of a polysimplicial complex on $A$. In general there exists a totally-geodesic subspace $A^{\prime} \subset A$ which is $W$-invariant and such that $A^{\prime} / W$ is compact. Therefore each alcove in $A$ is a product 
of simplices and a Euclidean subspace in $A$. Thus much of the discussion of Euclidean Coxeter complexes can be reduced to the case when $A / W$ is compact.

Remark 2.1. A triangulation of a fundamental alcove (chamber) in $A$ determines a $W$-invariant simplicial complex on $A$. Thus we can always think of $A$ as a simplicial complex.

A half-apartment in $A$ is the closure of a connected component of $A \backslash H$, where $H$ is a wall in $A$.

"Most" Coxeter complexes are associated with root systems as we describe below. Suppose that $R$ is a root system on a vector space $V$ (i.e., each element $\alpha \in R$ is a linear functional on $V$ ). The rank of $R$ is the number of simple roots in $R$, i.e., is the rank of the free abelian subgroup in $V^{*}$ generated by $R$. Let $A$ denote the Euclidean affine space corresponding to $V$. This data defines a finite Coxeter group $W_{\text {sph }}$, which is a reflection group generated by reflections in the hyperplanes $H_{\alpha}=\{x: \alpha(x)=0\}$. Weyl chambers of $W_{\text {sph }}$ are closures of the connected components of the complement to

$$
\bigcup_{\alpha \in R} H_{\alpha} .
$$

In what follows we fix a positive Weyl chamber $\Delta$, it determines the subset of positive roots $R^{+} \subset R$ and of simple roots $\Phi \subset R^{+}$. We also have the group of coweights $P\left(R^{\vee}\right)$ associated with $R$ :

$$
\lambda \in P\left(R^{\vee}\right) \Longleftrightarrow \alpha(\lambda) \in \mathbb{Z} \quad \text { for all } \alpha \in R .
$$

Let $W_{\text {aff }}$ denote the affine Coxeter group determined by the above data, this group is generated by reflections in the hyperplanes (affine walls)

$$
H_{\alpha, t}=\{\alpha(x)=t\}, \quad t \in \mathbb{Z} .
$$

Given a vector $\gamma \in \Delta$, we define the contragredient vector $\gamma^{*}$ as $w_{0}(-\gamma)$, where $w_{0}$ is the longest element of $W$. In other words, $\gamma^{*}$ is the intersection of the $W$-orbit $W \cdot(-\gamma)$ with the chamber $\Delta$.

The translation subgroup in $W_{\text {aff }}$ is the coroot lattice $Q\left(R^{\vee}\right)$, it is generated by the coroots $\alpha^{\vee}, \alpha \in R$. The group $P\left(R^{\vee}\right)$ is the normalizer of $W_{\text {aff }}$ in the group of translations $V$. Note that $V / P\left(R^{\vee}\right)$ is compact. The dimension of this quotient is the same as the dimension of $V$ provided that $\operatorname{rank}(R)$ equals the dimension of $V$.

The special vertices of a Euclidean Coxeter complex are the points whose stabilizer in $W_{\text {aff }}$ is isomorphic to $W_{\text {sph }}$. Equivalently, they are the points in the $P\left(R^{\vee}\right)$-orbit of the origin.

Remark 2.2. If $A / W_{\text {aff }}$ is compact, the vertices of ( $\left.A, W_{\text {aff }}\right)$ are the vertices of the polysimplicial complex determined by $W_{\text {aff }}$. 
Let $A^{(0)}$ denote the vertex set of $\left(A, W_{\text {aff }}\right)$, which consists of points of maximal intersection of walls in $A$. If $R$ spans $V^{*}$, the set $A^{(0)}$ equals the vertex set of the polysimplicial complex in $A$ defined by tessellation of $A$ via alcoves of $W_{\text {aff }}$.

Given a Coxeter complex $(A, W)$ and a point $x \in A$ we define a new Coxeter complex $\left(S_{x}, W_{x}\right)$ where $S_{x}$ is the unit tangent sphere at $x$ and $W_{x}$ is the stabilizer of $x$ in $W$.

For a nonzero vector $v \in V$ we let $\bar{v}:=v /|v|$ denote the normalization of $v$. We define rational elements of the unit sphere $S$ to be the unit vectors of the form

$$
\eta=\bar{v}, \quad v \in P\left(R^{\vee}\right) .
$$

The next lemma follows immediately from compactness of $V / P\left(R^{\vee}\right)$ :

Lemma 2.3. Rational points are dense in $S$.

Suppose that $(A, W)$ is a Euclidean Coxeter complex. A dilation of $(A, W)$ is a dilation $h$ (i.e. a composition of translation and similarity $v \mapsto \lambda v, \lambda>0$ ) in the affine space $A$ so that

$$
h W h^{-1} \subset W .
$$

We let $\operatorname{Dil}(A, W)$ denote the semigroup of dilations of the complex $(A, W)$. We will refer to the number $\lambda$ as the conformal factor of the dilation $h$.

Given a point $x \in A$ and a dilation $h \in \operatorname{Dil}(A, W)$, we can define a new spherical Coxeter complex $\left(S_{x}, W_{x}^{\prime}\right)$ on the unit tangent sphere $S_{x}$ at $x$ via pull-back

$$
W_{x}^{\prime}:=h^{*}\left(W_{t(x)}\right),
$$

where $W_{h(x)}$ is the stabilizer of $h(x)$ in $W$.

Definition 2.4. Suppose that $W$ is a finite Coxeter group acting on a vector space $V$. Define a (nontransitive) relation $\sim_{W}$ on $V \backslash\{0\}$ by

$$
\mu \sim_{W} v \Longleftrightarrow \mu, v \text { belong to the same Weyl chamber of } W .
$$

We will frequently omit the subscript $W$ in this notation.

Definition 2.5 ([L2], p. 514). We say that nonzero vectors $v, \mu \in V$ satisfy $v \triangleright_{W} \mu$ (for short, $v \triangleright \mu$ ) if for each positive root $\alpha$,

$$
\alpha(v)<0 \Longrightarrow \alpha(\mu) \leq 0 .
$$

Lemma 2.6. Suppose that $v, \mu \in P\left(R^{\vee}\right), w \in W=W_{\text {aff }}$ is such that $w(v)=\mu$. Then

$$
\mu-v \in Q\left(R^{\vee}\right) .
$$


Proof. The mapping $w$ is a composition of reflections $\tau_{i} \in W$. Therefore it suffices to prove the assertion in case when $w$ is a reflection $\tau$. This reflection is a composition of a translation $t$ and a reflection $\sigma \in W_{o}$. The translation $t$ belongs to the translation subgroup $Q\left(R^{\vee}\right)$ of $W$, therefore it suffices to consider the case when $\tau=\sigma \in W_{o}$. Then $\tau=\tau_{\beta}$, where $\beta$ is a root and we have

$$
\mu-v=-\beta(v) \beta^{\vee} .
$$

Since $\beta(v) \in \mathbb{Z}$ and $\beta^{\vee} \in Q\left(R^{\vee}\right)$, the assertion follows.

2.2. Paths. Suppose that $A, V, W_{\text {aff }}$, etc., are as in the previous section.

Let $\widetilde{\mathcal{P}}$ denote the set of all piecewise-linear paths $p:[a, b] \rightarrow V$. We will be identifying paths that differ by orientation-preserving re-parameterizations $[a, b] \rightarrow$ $\left[a^{\prime}, b^{\prime}\right]$. Accordingly, we will always (re)parameterize a piecewise-linear path with constant speed. We let $p_{-}^{\prime}(t), p_{+}^{\prime}(t)$ denote the derivatives of the function $p$ from the left and from the right. The space $\widetilde{\mathcal{P}}$ will be given the topology of uniform convergence.

If $p, q:[0,1] \rightarrow A$ are piecewise-linear paths in a simplicial complex such that $p(1)=q(0)$, we define their composition $r=p \cup q$ by

$$
r(t)= \begin{cases}p(t), & t \in[0,1], \\ q(t-1), & t \in[1,2] .\end{cases}
$$

Let $\mathcal{P} \subset \widetilde{\mathcal{P}}$ denote the set of paths $p:[0,1] \rightarrow V$ such that $p(0)=0$. Given a path $p \in \mathcal{P}$ we let $p^{*} \in \mathcal{P}$ denote the reverse path

$$
p^{*}(t)=p(1-t)-p(1) .
$$

For a vector $\lambda \in V$ define a geodesic path $\pi_{\lambda} \in \mathcal{P}$ by

$$
\pi_{\lambda}(t)=t \lambda, \quad t \in[0,1] .
$$

Given two paths $p_{1}, p_{2} \in \mathcal{P}$ define their concatenation $p=p_{1} * p_{2}$ by

$$
p(t)= \begin{cases}c p_{1}(2 t), & t \in[0,1 / 2], \\ p_{1}(1)+p_{2}(2 t-1), & t \in[1 / 2,1] .\end{cases}
$$

Suppose that $p \in \mathscr{P}$ and $J=[a, b]$ is nondegenerate subinterval in $I=[0,1]$. We will use the notation $p \mid J \in \widetilde{\mathcal{P}}$ to denote the function-theoretic restriction of $p$ to $[a, b]$. We will use the notation $\left.p\right|_{J}$ to denote the path in $\mathcal{P}$ obtained from $p \mid J$ by precomposing $p \mid J$ with an increasing linear bijection $\ell: I \rightarrow J$ and post-composing it with the translation by the vector $-p(a)$. 
Fix a positive Weyl chamber $\Delta \subset V$; this determines the set of positive roots $R^{+} \subset R$, the set of simple roots $\Phi \subset R^{+}$. We define the subset $\mathcal{P}^{+} \subset \mathcal{P}$ consisting of the paths whose image is contained in $\Delta$.

For a path $p \in \mathcal{P}$ and a positive root $\alpha \in R^{+}$define the height function

$$
h_{\alpha}(t)=\alpha(p(t))
$$

on $[0,1]$. Let $m_{\alpha}=m_{\alpha}(p) \in \mathbb{R}$ denote the minimum of $h_{\alpha}$. Clearly $m_{\alpha}(p) \leq 0$ for all $p \in \mathcal{P}$. We define the set of "integral paths"

$$
\mathcal{P}_{\mathbb{Z}}:=\left\{p \in \mathcal{P}: m_{\alpha}(p) \in \mathbb{Z} \text { for all } \alpha \in \Phi\right\} .
$$

More restrictively, we define the set $\mathcal{P}_{\mathbb{Z}, \text { loc }}$ of paths $p \in \mathcal{P}$ which satisfy the following local integrality condition:

For each simple root $\alpha \in \Phi$ the function $h_{\alpha}$ takes integer values at the points of local minima.

2.3. The saturation factors associated to a root system. In this section we define and compute saturation factors associated with root systems. Let $o \in A$ be a special vertex, which we will identify with $0 \in V$.

Definition 2.7. We define the saturation factor $k_{R}$ for the root system $R$ to be the least natural number $k$ such that $k \cdot A^{(0)} \subset P\left(R^{\vee}\right) \cdot o$. The numbers $k_{R}$ for the irreducible root systems are listed in Table 1 .

Note that the condition that $k \cdot A^{(0)} \subset P\left(R^{\vee}\right) \cdot o$ is equivalent to that each point of $k \cdot A^{(0)}$ is a special vertex.

Below we explain how to compute the saturation factors $k_{R}$ following [KLM3]. First of all, it is clear that if the root system $R$ is reducible and $R_{1}, \ldots, R_{s}$ are its irreducible components, then $k_{R}=\operatorname{lcm}\left(k_{R_{1}}, \ldots, k_{R_{S}}\right)$, where $1 \mathrm{~cm}$ stands for the least common multiple. Henceforth we can assume that the system $R$ is reduced, irreducible and has rank $n=\operatorname{dim}(V)$. Then the affine Coxeter group $W_{\text {aff }}$ acts cocompactly on $A$ and its fundamental domain (a Weyl alcove) is a simplex.

Let $\left\{\alpha_{1}, \ldots, \alpha_{\ell}\right\}$ be the collection of simple roots in $R$ (corresponding to the positive Weyl chamber $\Delta$ ) and $\theta$ be the highest root. Then

$$
\theta=\sum_{i=1}^{\ell} m_{i} \alpha_{i}
$$

We have

Lemma 2.8 ([KLM3], Section 2). $k_{R}=\operatorname{lcm}\left(m_{1}, \ldots, m_{n}\right)$.

Below is the list of saturation factors: 
Table 1

\begin{tabular}{|l|l|r|}
\hline Root system & $\theta$ & $k_{R}$ \\
\hline$A_{\ell}$ & $\alpha_{1}+\cdots+\alpha_{\ell}$ & 1 \\
\hline$B_{\ell}$ & $\alpha_{1}+2 \alpha_{2}+\cdots+2 \alpha_{\ell}$ & 2 \\
\hline$C_{\ell}$ & $2 \alpha_{1}+2 \alpha_{2}+\cdots+2 \alpha_{\ell-1}+\alpha_{\ell}$ & 2 \\
\hline$D_{\ell}$ & $\alpha_{1}+\alpha_{2}+\alpha_{3}+2 \alpha_{4}+\cdots+2 \alpha_{\ell}$ & 2 \\
\hline$G_{2}$ & $3 \alpha_{1}+2 \alpha_{2}$ & 6 \\
\hline$F_{4}$ & $2 \alpha_{1}+3 \alpha_{2}+4 \alpha_{3}+2 \alpha_{4}$ & 12 \\
\hline$E_{6}$ & $\alpha_{1}+\alpha_{2}+2 \alpha_{3}+2 \alpha_{4}+2 \alpha_{5}+3 \alpha_{6}$ & 6 \\
\hline$E_{7}$ & $\alpha_{1}+2 \alpha_{2}+2 \alpha_{3}+2 \alpha_{4}+3 \alpha_{5}+3 \alpha_{6}+4 \alpha_{7}$ & 12 \\
\hline$E_{8}$ & $2 \alpha_{1}+2 \alpha_{2}+3 \alpha_{3}+3 \alpha_{4}+4 \alpha_{5}+4 \alpha_{6}+5 \alpha_{7}+6 \alpha_{8}$ & 60 \\
\hline
\end{tabular}

2.4. Buildings. Our discussion of buildings follows $[\mathrm{KL}]$. We refer the reader to [Br], [Ron], [Rou] for the more combinatorial discussion.

Fix a spherical or Euclidean (discrete) Coxeter complex $(A, W)$, where $A$ is a Euclidean space $E$ or a unit sphere $S$ and $W=W_{\text {aff }}$ or $W=W_{\text {sph }}$ is a discrete Euclidean or a spherical Coxeter group acting on $A$.

A metric space $Z$ is called geodesic if any pair of points $x, y$ in $Z$ can be connected by a geodesic segment $\overline{x y}$.

Let $Z$ be a metric space. A geometric structure on $Z$ modeled on $(A, W)$ consists of an atlas of isometric embeddings $\varphi: A \hookrightarrow Z$ satisfying the following compatibility condition: For any two charts $\varphi_{1}$ and $\varphi_{2}$, the transition map $\varphi_{2}^{-1} \circ \varphi_{1}$ is the restriction of an isometry in $W$. The charts and their images, $\varphi(A)=a \subset Z$, are called apartments. We will sometimes refer to $A$ as the model apartment. We will require that there are plenty of apartments in the sense that any two points in $Z$ lie in a common apartment. All $W$-invariant notions introduced for the Coxeter complex $(A, W)$, such as rank, walls, singular subspaces, chambers etc., carry over to geometries modeled on $(A, W)$. If $a, a^{\prime} \subset X$ are alcoves (in the Euclidean case) or chambers (in the spherical case) then there exists an apartment $A^{\prime} \subset X$ containing $a \cup a^{\prime}$ : Just take regular points $x \in a, x^{\prime} \in a^{\prime}$ and an apartment $A^{\prime}$ passing through $x$ and $x^{\prime}$.

A geodesic metric space $Z$ is said to be a CAT $(\kappa)$-space if geodesic triangles in $Z$ are "thinner" than geodesic triangles in a simply-connected complete surface of the constant curvature $\kappa$. We refer the reader to [Ba] for the precise definition. Suppose that $Z$ is a (non-geodesic) metric space with the discrete metric:

$$
d(x, y)=\pi \Longleftrightarrow x \neq y .
$$

We will regard such a space as a CAT(1) space as well. 
Definition 2.9. A spherical building is a CAT(1)-space modeled on a spherical Coxeter complex.

Spherical buildings have a natural structure of polysimplicial piecewise spherical complexes. We prefer the geometric to the combinatorial view point because it appears to be more appropriate in the context of this paper.

Definition 2.10. A Euclidean building is a CAT(0)-space modeled on a (discrete) Euclidean Coxeter complex.

A building is called thick if every wall is an intersection of apartments. A nonthick building can always be equipped with a natural structure of a thick building by reducing the Coxeter group [KL].

Let $\mathbb{K}$ be a local field with a (discrete) valuation $v$ and valuation ring $\mathcal{O}$. Throughout this paper, we will be working with $\mathbb{Q}$-split reductive algebraic groups (i.e., Chevalley groups) $\underline{G}$ over $\mathbb{Z}$. We refer the reader to [B], [D] for a detailed discussion of such groups. A reader can think of $\mathbb{K}=\mathbb{Q}_{p}$ (the $p$-adic numbers) and a classical Chevalley group $\underline{G}$, e.g., $\operatorname{GL}(n)$ or $\operatorname{Sp}(n)$, in which case Bruhat-Tits buildings below can be described rather explicitly; see, e.g., [G].

Given a group $\underline{G}$ as above, we get a nonarchimedean Lie group $G=\underline{G}(\mathbb{K})$, to which we can associate a Euclidean building (a Bruhat-Tits building) $X=X_{G}$. We refer the reader to [BT], [KLM3] and [Rou] for more detailed discussion of the properties of $X$. Here we only recall that:

(1) $X$ is thick and locally compact.

(2) $X$ is modeled on a Euclidean Coxeter complex $\left(A, W_{\text {aff }}\right)$ whose dimension equals the rank of $\underline{G}$, and the root system is isomorphic to the root system of $\underline{G}$.

(3) $X$ contains a special vertex $o$ whose stabilizer in $G$ is $\underline{G}(\mathcal{O})$.

Example 2.11. Let $X$ be a (discrete) Euclidean building, consider the spaces of directions $\Sigma_{x} X$. We will think of this space as the space of germs of non-constant geodesic segments $\overline{x y} \subset X$. As a polysimplicial complex $\Sigma_{x} X$ is just the link of the point $x \in X$. The space of directions has the structure of a spherical building modeled on $\left(S, W_{\mathrm{sph}}\right)$, which is thick if and only if $x$ is a special vertex of $X[\mathrm{KL}]$. The same applies in the case when $X$ is a spherical building.

If $X$ is a Euclidean building modeled on $(A, W)$, for each point $x \in X$ the space of directions $\Sigma_{x}(X)$ has two structures of a spherical building:

(1) The restricted building structure which is modeled on the Coxeter complex $\left(S, W_{x}\right)$, where $S=S_{x}(A)$ is the unit tangent sphere at $x$ and $W_{x}$ is the stabilizer of $x$ in the Coxeter group $W$. This building structure is thick. 
(2) The unrestricted building structure which is modeled on the Coxeter complex $\left(S, W_{\text {sph }}\right.$ ), where $S=S_{x}(A)$ is the unit tangent sphere at $x$ and $W_{\text {sph }}$ is the linear part of the affine Coxeter group $W_{\text {aff }}$. This building structure is not thick, unless $x$ is a special vertex.

Let $B$ be a spherical building modeled on a spherical Coxeter complex $\left(S, W_{\mathrm{sph}}\right)$. We say that two points $x, y \in B$ are antipodal, if $d(x, y)=\pi$; equivalently, they are antipodal points in an apartment $S^{\prime} \subset B$ containing both $x$ and $y$. The quotient map $S \rightarrow S / W_{\text {sph }} \cong \Delta_{\text {sph }}$ induces a canonical projection $\theta: B \rightarrow \Delta_{\text {sph }}$ folding the building onto its model Weyl chamber. The $\theta$-image of a point in $B$ is called its type.

Remark 2.12. To define $\theta(x)$, pick an apartment $S^{\prime}$ containing $x$ and a chart $\phi: S \rightarrow S^{\prime}$. Then $\theta(x)$ is the projection of $\phi^{-1}(x)$ to $S / W_{\text {sph }} \cong \Delta_{\text {sph }}$. We note that this is clearly independent of $S^{\prime}$ and $\phi$.

Lemma 2.13. (1) If $h: A \rightarrow A^{\prime}$ is an isomorphism of apartments in $B$ (i.e., $\left.\phi^{\prime-1} \circ h \circ \phi \in W\right)$ then $\theta \circ h=\theta$.

(2) If $x, x^{\prime} \in B$ which belong to apartments $A, A^{\prime}$ respectively and $-x \in A$, $-x^{\prime} \in A^{\prime}$ are antipodal to $x, x^{\prime}$, then $\theta(x)=\theta\left(x^{\prime}\right)$ implies $\theta(-x)=\theta\left(-x^{\prime}\right)$.

Proof. (1) is obvious, so we prove (2). Pick an isomorphism $h: A \rightarrow A^{\prime}$. Then (since $\left.\theta(x)=\theta\left(x^{\prime}\right)\right)$ there exists $w \in W \curvearrowright A^{\prime}$ such that $w(h(x))=x^{\prime}$. Hence $w \circ h(-x)=-w \circ(x)=-x^{\prime}$. The claim now follows from (1).

We will regard $n$-gons $P$ in a building $X$ as maps $v:\{1, \ldots, n\} \rightarrow X, v(i)=x_{i}$, where $x_{i}$ will be the vertices of $P$. If $\operatorname{rank}(X) \geq 1$ we can connect the consecutive vertices $x_{i}, x_{i+1}$ by shortest geodesic segments $\overline{x_{i} x_{i+1}} \subset X$ thus creating a geodesic polygon $\left[x_{1}, x_{2}, \ldots, x_{n}\right]$ in $X$ with the edges $\overline{x_{i} x_{i+1}}$. Observe that in case $x_{i}, x_{i+1}$ are antipodal, the edge $\overline{x_{i} x_{i+1}}$ is not unique.

We say that two subsets $F, F^{\prime}$ in a building $X$ are congruent if there exist apartments $A, A^{\prime}$ in $X$ containing $F, F^{\prime}$ respectively, and an isomorphism $A \rightarrow A^{\prime}$ of Coxeter complexes which carries $F$ to $F^{\prime}$.

Convention 2.14. Suppose that $X$ is a spherical building. We will be considering only those geodesic triangles $T$ in $X$ for which the length of each geodesic side of $T$ is $\leq \pi$.

Let $X, Y$ be buildings and $f: X \rightarrow Y$ a continuous map satisfying the following: For each alcove (in Euclidean case) or spherical chamber (in the spherical case) $a \subset X$, the image $f(a)$ is contained in an apartment of $Y$ and the restriction $f \mid a$ is an isometry or similarity. Then we call $f$ differentiable and define the derivative $d f$ of $f$ as follows. Given a point $x \in X$ and $y=f(x)$, the derivative $d f_{x}$ is a map $\Sigma_{x}(X) \rightarrow \Sigma_{y}(Y)$. For each $\xi \in \Sigma_{x}(X)$ let $\overline{x z} \subset X$ be a geodesic segment 
whose interior consists of regular points only and so that $\xi$ is the unit tangent vector to $\overline{x z}$. Then $f$ sends $\overline{x z}$ to a nondegenerate geodesic segment $\overline{y f(z)}$ contained in an apartment $A \subset Y$. Then we let $d f_{x}(\xi) \in \Sigma_{y}(Y)$ be the unit tangent vector to $\overline{y f(z)}$.

We will be also using the above definition in the setting when a building $Y$ is a Euclidean Coxeter complex $(A, W)$ and $h \in \operatorname{Dil}(A, W)$. Then after letting $X:=$ $h^{*}(A, W)$ we get an isometry $h: X \rightarrow Y$.

Convention 2.15. Throughout the paper we will be mostly using roman letters $x, y$, $z$, etc., to denote points in Euclidean buildings and Greek letters $\xi, \eta, \zeta$, etc., to denote points in spherical buildings. Sometimes however (e.g., in Section 4.2) we will be working simultaneously with a spherical building $X$ and its links $\Sigma_{x}(X)$, which are also spherical buildings. In this case we will use roman letters for points in $X$ and Greek letters for points in $\Sigma_{x}(X)$.

2.5. Generalized distances and lengths in buildings. Let $(A, W)$ be a spherical or Euclidean Coxeter complex. The complete invariant of a pair of points $(x, y) \in$ $A^{2}$ with respect to the action $W \curvearrowright A$, is its image $d_{\text {ref }}(x, y)$ under the canonical projection to $A \times A / W$. Following [KLM2] we call $d_{\text {ref }}(x, y)$ the refined distance from $x$ to $y$. This notion carries over to buildings modeled on the Coxeter complex $(A, W)$ : For a pair of points $(x, y)$ pick an apartment $A^{\prime}$ containing $x, y$ and, after identifying $A^{\prime}$ with the model apartment $A$, let $d_{\text {ref }}(x, y)$ be the projection of this pair to $A \times A / W$.

If points $\xi, \eta$ in a spherical building are antipodal we will use $\pi$ for the refined distance $d_{\text {ref }}(\xi, \eta)$. This does not create much ambiguity since given apartment contains unique point antipodal to $\xi$.

In the case of Euclidean Coxeter complexes there is an extra structure associated with the concept of refined length. Given a Euclidean Coxeter complex $\left(A, W_{\text {aff }}\right)$, pick a special vertex $o \in A$. Then we can regard $A$ as a vector space $V$, with the origin $0=o$. Let $\Delta \subset A$ denote a Weyl chamber of $W_{\mathrm{sph}}$, the tip of $\Delta$ is at $o$.

Then following [KLM2], we define the $\Delta$-distance between points of $\left(A, W_{\text {aff }}\right)$ by composing $d_{\text {ref }}$ with the natural forgetful map

$$
A \times A / W_{\mathrm{aff}} \rightarrow A / W_{\mathrm{sph}} \cong \Delta .
$$

To compute the $\Delta$-distance $d_{\Delta}(x, y)$ we regard the oriented geodesic segment $\overline{x y}$ as a vector in $V$ and project it to $\Delta$. Again, the concept of $\Delta$-distance, carries over to the buildings modeled on $\left(A, W_{\text {aff }}\right)$.

Definition 2.16. Let $X$ be a thick Euclidean building. Define the set $D_{n}(X) \subset \Delta^{n}$ of $\Delta$-side lengths which occur for geodesic $n$-gons in $X$.

It is one of the results of [KLM2] that $D_{n}:=D_{n}(X)$ is a convex homogeneous polyhedral cone in $\Delta^{n}$, which depends only on $\left(A, W_{\mathrm{sph}}\right)$. The polyhedron $D_{3}$ in 
Theorem 1.4 is the polyhedron $D_{3}(X)$. The set of stability inequalities defining $D_{n}$ is determined in [KLM1] and [BS].

Theorem 2.17 ([KLM3], Corollary 8.4). Let $X$ be a thick Euclidean building modeled on $\left(A, W_{\mathrm{aff}}\right)$. Suppose that $\alpha, \beta, \gamma \in P\left(R^{\vee}\right), \alpha+\beta+\gamma \in Q\left(R^{\vee}\right)$ and $(\alpha, \beta, \gamma) \in D_{3}(X)$. Then there exists a geodesic triangle $T \subset X$ whose vertices are vertices of $X$ and the $\Delta$-side lengths are $\alpha, \beta, \gamma$.

Suppose that $p$ is a piecewise-linear path in a Euclidean building $X$. We say that $p$ is a billiard path if for each $t, s \in[0,1]$ the tangent vectors $p^{\prime}(t), p^{\prime}(s)$ have the same projection to the chamber $\Delta$ in the model apartment. If $p$ is a path which is the composition

$$
\overline{x_{0} x_{1}} \cup \cdots \cup \overline{x_{m-1} x_{m}}
$$

of geodesic paths, then the $\Delta$-length of $p$ is defined as

$$
\operatorname{length}_{\Delta}(p):=\sum_{i=1}^{m} d_{\Delta}\left(x_{i-1}, x_{i}\right)
$$

where $d_{\Delta}(x, y)$ is the $\Delta$-distance from $x$ to $y$.

Each piecewise-linear path $p$ admits a unique representation

$$
p=p_{1} \cup \cdots \cup p_{n}
$$

as a composition of maximal billiard subpaths so that

$$
\lambda_{i}=\text { length }_{\Delta}\left(p_{i}\right)
$$

We define

$$
\text { length }_{\Delta}(p):=\underline{\lambda}=\left(\lambda_{1}, \ldots, \lambda_{n}\right) .
$$

Clearly, length ${ }_{\Delta}(p)$ is the sum of the vector components of $\underline{\lambda}$.

2.6. The Hecke ring. In this section we review briefly the definition of spherical Hecke rings and their relation to the geometry of Euclidean buildings; see [Gro], [KLM3] for more details.

Let $\mathbb{K}$ denote a locally compact field with discrete valuation $v$, and (necessarily) finite residue field of the order $q$. Let $\mathcal{O}$ be the subring of $\mathbb{K}$ consisting of elements with nonnegative valuation. Choose a uniformizer $\pi \in \mathcal{O}$.

Consider a connected reductive algebraic group $\underline{G}$ over $\mathbb{K}$. We fix a maximal split torus $\underline{T} \subset \underline{G}$ defined over $\mathcal{O}$. We put $G:=\underline{G}(\mathbb{K}), K:=\underline{G}(\mathcal{O})$ and $T:=\underline{T}(\mathbb{K})$. We let $\underline{B} \subset \underline{G}$ be a Borel subgroup normalized by $\underline{T}$ and set $B:=\underline{B}(\mathbb{K})$.

Let $X$ denote the Bruhat-Tits building associated with the group $\underline{G} ; o \in X$ is a distinguished special vertex stabilized by the compact subgroup $K$. 
We also have free abelian cocharacter group $X_{*}(\underline{T})$ of $\underline{T}$ whose rank equals $\operatorname{dim}(\underline{T})$. This group contains the set of coroots $R^{\vee}$ of the group $\underline{G}$. The roots are the characters of $\underline{T}$ that occur in the adjoint representation on the Lie algebra of $\underline{G}$. The subset $R^{+}$of the roots that occur in representation on the Lie algebra of $\underline{B}$ forms a positive system and the indecomposable elements of that positive system form a system of simple roots $\Phi$. We let $W$ denote the corresponding (finite) Weyl group.

The set of positive roots $\Phi$ determines a positive Weyl chamber $\Pi^{+}$in $X_{*}(\underline{T})$, by

$$
\Pi^{+}=\left\{\lambda \in X_{*}(\underline{T}):\langle\lambda, \alpha\rangle \geq 0, \alpha \in \Phi\right\} .
$$

This chamber is a fundamental domain for the action of $W$ on $X_{*}(\underline{T})$.

We define a partial ordering on $\Pi^{+}$by $\lambda \succ \mu$ iff the difference $\lambda-\mu$ is a sum of positive coroots.

Definition 2.18. The (spherical) Hecke ring $\mathscr{H}=\mathscr{H}_{G}$ is the ring of all locally constant, compactly supported functions $f: G \rightarrow \mathbb{Z}$ which are $K$-biinvariant. The multiplication in $\mathscr{H}$ is by the convolution

$$
f \star g(z)=\int_{G} f(x) \cdot g\left(x^{-1} z\right) d x
$$

where $d x$ is the Haar measure on $G$ giving $K$ volume 1 .

The ring $\mathscr{H}$ is commutative and associative (see, e.g., [Gro]). For $\lambda \in X_{*}(\underline{T})$ let $c_{\lambda}$ be the characteristic function of the corresponding $K$-double coset $\lambda(\pi) \in K \backslash G / K$. Then the functions $c_{\lambda}, \lambda \in \Delta$ freely generate $\mathscr{H}$ as an (additive) abelian group. Deep result of Satake [Sat] relates the Hecke ring of $G$ and the representation ring of $G^{\vee}$.

The structure constants for $\mathscr{H}$ are defined by the formula

$$
c_{\lambda} \star c_{\mu}=\sum_{\nu} m_{\lambda, \mu}(v) c_{v}=c_{\lambda+\mu}+\sum m_{\lambda, \mu}(v) c_{v},
$$

where the last sum is taken over all $v \in \Pi^{+}$such that $\lambda+\mu \succ v$ and therefore is finite.

It turns out that the structure constants $m_{\lambda, \mu}(v)$ are nonnegative integers which are polynomials in $q$ with integer coefficients. These constants are determined by the geometry of the building as follows. Given $\alpha, \beta, \gamma$ let $\mathcal{T}=\mathcal{T}_{\alpha, \beta}(\gamma)$ denote the (finite) set of geodesic triangles $[o, x, y]$ in the building $X$ which have the $\Delta$-side lengths $\alpha$, $\beta, \gamma^{*}$, so that $y$ is the projection of the point

$$
\gamma(\pi) \in T \subset G
$$

into $X$ under the map $g \mapsto g \cdot o$. Recall that $\gamma$ as a cocharacter and therefore it defines a homomorphism $\mathbb{K}^{*} \rightarrow T$. 
Theorem 2.19 ([KLM3], Theorem 9.11). $m_{\alpha, \beta}(\gamma)$ equals the cardinality of $\mathcal{T}$.

Remark 2.20. Instead of relating geometry of locally compact buildings to representation theory of $G^{\vee}$ as it is done in this paper, one can use the non-locally compact building associated with the group $\underline{G}(\mathbb{C}((t)))$, as it is done, for instance, in [GL]. It was shown in [KLM2] that the choice of a field with discrete valuation (or, even, the entire Euclidean building) is irrelevant as far as the existence of triangles with the given $\Delta$-side lengths is concerned: What is important is the affine Weyl group. For our purposes, moreover, it is more convenient to work with local fields and locallycompact buildings. In particular, it allows us to compare structure constants of Hecke and representation rings.

\section{Chains}

3.1. Absolute chains. Let $R$ be a root system on a Euclidean vector space $V, W=$ $W_{\text {sph }}$ be the finite Coxeter group associated with $R$, let $W_{\text {aff }}$ denote the affine Coxeter group associated to $R$. Our root system $R$ is actually the coroot system for the one considered by Littelmann in [L2]. Accordingly, we will switch weights to coweights, etc. We pick a Weyl chamber $\Delta$ for $W$, this determines the positive roots and the simple roots in $R$. Let $-\Delta$ denote the negative chamber.

We get the Euclidean Coxeter complex $\left(A, W_{\text {aff }}\right)$, where $A=V$ and the spherical Coxeter complex $(S, W)$ where $S$ is the unit sphere in $V$. By abusing notation we will also use the notation $\Delta,-\Delta$ for the positive and negative chambers in $(S, W)$. We will use the notation $(A, W,-\Delta)$ for a Euclidean/spherical Coxeter complex with chosen negative chamber. More generally, we will use the notation $\left(A, W_{\text {aff }}, a\right)$ for a Euclidean Coxeter complex with chosen alcove $a$.

Definition 3.1. A $W$-chain in $(A, W,-\Delta)$ is a finite sequence $\left(\eta_{0}, \ldots, \eta_{m}\right)$ of nonzero vectors in $V$ so that for each $i=1, \ldots, m$ there exists a positive root $\beta_{i} \in R^{+}$ such that the corresponding reflection $\tau_{i}:=\tau_{\beta_{i}} \in W$ satisfies

(1) $\tau_{i}\left(\eta_{i-1}\right)=\eta_{i}$;

(2) $\beta_{i}\left(\eta_{i-1}\right)<0$.

Sometimes we will refer to a chain as a $(V, W,-\Delta)$-chain to emphasize the choice of $V, W$ and $\Delta$. When the choice of $W$ is clear we will frequently refer to $W$-chains as chains.

Remark 3.2. One could call these chains $(V, W, \Delta)$-chains instead, but this definition would not generalize to affine chains.

Recall that $\tau_{i}$ is the reflection in the wall $H_{i}=\left\{\beta_{i}=0\right\}$. More geometrically one can interpret the condition (2) by saying that the wall $H_{i}$ separates the negative 
chamber $-\Delta$ from the vector $\eta_{i}$. In other words, the reflection $\tau_{i}$ moves the vector $\eta_{i-1}$ "closer" to the positive chamber.

The concept of a chain generalizes naturally to Euclidean Coxeter complexes $\left(A, W_{\text {aff }}\right)$. Pick an alcove $a$ in $A$.

Definition 3.3. An affine chain in $\left(A, W_{\text {aff }}, a\right)$ is a finite sequence $\left(\eta_{0}, \ldots, \eta_{m}\right)$ of elements in $A$ so that for each $i=1, \ldots, m$ there exists a reflection $\tau_{i} \in W_{\text {aff }}$ such that

(1) $\tau_{i}\left(\eta_{i-1}\right)=\eta_{i}$.

(2) The hyperplane $H_{i} \subset A$ fixed by $\tau_{i}$ separates $a$ from $\eta_{i}$.

We now return to the chains as in Definition 3.1. By restricting vectors $\eta_{i}$ to have unit length we define chains in the spherical Coxeter complex; see Figure 2.

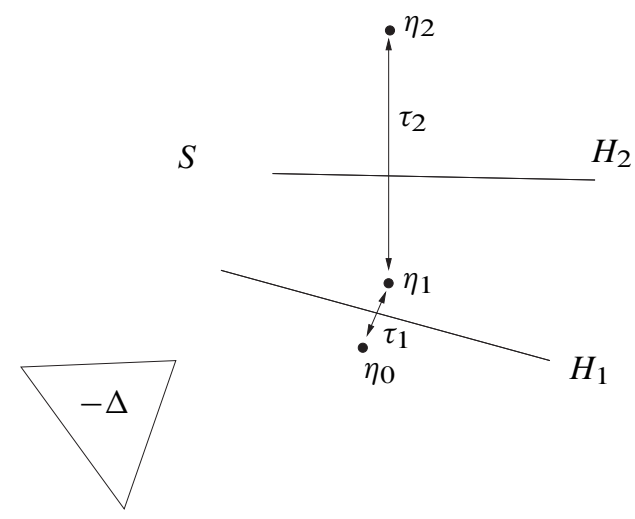

Figure 2. A chain.

Definition 3.4. The points $\eta_{i}$ as in Definition 3.1 are called vertices of the chain. We say that the chain begins at $\eta_{0}$ and ends at $\eta_{m}$, or that this chain is from $\eta_{0}$ to $\eta_{m}$. We refer to a subsequence $\left(\eta_{i}, \eta_{i+1}, \ldots, \eta_{m}\right)$ as a tail of the chain.

We will refer to the number $m$ as the length of the chain. A chain $\left(\eta_{i}\right)$ is called simple if it has length 1 . Set

$$
\operatorname{dist}_{W}(\nu, \mu)=\operatorname{dist}(\nu, \mu)
$$

to be the maximal length $m$ of a $W$-chain which begins at $v$ and ends at $\mu$.

Given a chain $\left(\eta_{0}, \ldots, \eta_{m}\right)$ we define a subdivision of this chain to be a new chain in $(A, W)$ which is still a chain from $\eta_{0}$ to $\eta_{m}$ and which contains all the vertices of the original chain.

The concept of chain determines a partial order on the $W$-orbits in $V$ : 
Definition 3.5 ([L2], p. 509). For a pair of nonzero vectors $v, \mu \in V$ which belong to the same $W$-orbit, write $v \geq W \mu$ (or simply $v \geq \mu$ ) if there exists a $W$-chain from $v$ to $\mu$. Accordingly, $v>\lambda$ if $v \geq \lambda$ and $v \neq \lambda$.

Lemma 3.6. Suppose that $v \geq \mu$ and $\alpha$ is a positive root such that $\alpha(\mu) \leq 0$. Then

$$
v \geq \tau_{\alpha}(\mu)
$$

Proof. If $\alpha(\mu)=0$ then $\tau_{\alpha}(\mu)=\mu$ and there is nothing to prove. Thus we assume that $\alpha(\mu)<0$. Consider a chain $\left(v=v_{0}, \ldots, v_{s}=\mu\right)$. Then, since $\alpha(\mu)<0$, we also have $\alpha\left(\tau_{\alpha}(\mu)\right)>0$ and thus we get a longer chain

$$
\left(v=v_{0}, \ldots, v_{s}=\mu, \tau(\mu)\right) .
$$

The word metric $d_{W}$ on the finite Coxeter group $W$ defines the length function

$$
\ell: W \cdot \lambda \rightarrow \mathbb{N}
$$

by

$$
\ell(\mu):=\min \left\{d_{W}(w, 1): w \in W, w^{-1}(\mu) \in \Delta\right\} .
$$

Proposition 3.7. If $v>\mu$ then $\ell(v)>\ell(\mu)$.

Proof. It suffices to prove the assertion in the case when

$$
\mu=\tau(v), \quad \tau=\tau_{\beta},
$$

where $\beta$ is a positive root, $\beta(v)<0, \beta(\mu)>0$. If $W \cong \mathbb{Z} / 2$ the assertion is clear, so we suppose that it is not the case. Then we can embed the Cayley graph $\Gamma$ of $W$ as a dual graph to the tessellation of $V$ by the Weyl chambers of $W$. Suppose that $v \in w^{-1}(\Delta)$, then the wall $H_{\beta}=\{\beta=0\}$ separates $w^{-1}(\Delta)$ from $\Delta$, where $w \in W$ is the shortest element such that $w(v) \in \Delta$. Let $p:[0,1] \rightarrow \Gamma$ denote the shortest geodesic from 1 to $w$ in $\Gamma$. The path $p$ crosses the wall $H$ at a point $x=p(T)$. We construct a new path $q$ by

$$
q|[0, T]=p|[0, T], \quad q|[T, 1]=w \circ p|[T, 1] .
$$

The path $q$ connects $1 \in \Delta$ to the Weyl chamber $\tau w(\Delta)$ containing $\mu$. This path has a break point at $x$, which is not a vertex of the Cayley graph. Therefore, by eliminating the backtracking of $q$ at $x$, we obtain a new path which connects 1 to $\tau w(\Delta)$ and whose length is one less than the length of $p$.

Corollary 3.8. The length of a chain in $V$ does not exceed the diameter of the Cayley graph of $W$. 
Corollary 3.9. Suppose that $v \in \Delta$ and $v \geq \mu$. Then $\mu=v$.

Proof. Since $v \in \Delta, \ell(v)=0$. Hence by Proposition 3.7, $\ell(\mu)=0$, which implies that $\mu=v$.

Lemma 3.10. Suppose that $\nu=w(\mu) \neq 0$ for some $w \in W$ and $\mu \triangleright v$. Then $v \geq \mu$.

Proof. Let $\Delta$ be the positive Weyl chamber. Suppose that $\Delta_{0}$ is a chamber containing $v$ and $\Delta^{\prime}$ is a chamber containing $\mu$. These chambers are non-unique, but we can choose them in such a way that

$$
\xi^{\prime} \triangleright \xi
$$

for all $\xi \in \Delta_{0}, \xi^{\prime} \in \Delta^{\prime}$. In other words, if a wall separates $\Delta^{\prime}$ from $\Delta$ then it also separates $\Delta_{0}$ from $\Delta$. Let

$$
\left(\Delta_{0}, \Delta_{1}, \ldots, \Delta_{m}=\Delta^{\prime}\right)
$$

be a gallery of Weyl chambers, i.e., for each $i, \Delta_{i} \cap \Delta_{i+1}$ is a codimension 1 face $F_{i}$ of $\Delta_{i}, \Delta_{i+1}$. We choose this gallery to have the shortest length, i.e., the number $m$ is minimal. Let $H_{i}$ be the wall containing $F_{i}$ and $\tau_{i}$ be the reflection in $H_{i}$. We claim that the sequence

$$
v=\eta_{0}, \quad \eta_{1}=\tau_{1}\left(\eta_{0}\right), \ldots, \mu=\eta_{m}=\tau_{m}\left(\eta_{m-1}\right),
$$

after deletion of equal members, is a chain.

Our proof is by induction on $m$. If $m=0$ and $v=\mu$, there is nothing to prove. Suppose the assertion holds for $m-1$, let us show it for $m$. We claim that $\xi_{1} \triangleright \xi_{0}$ for all points $\xi_{1} \in \Delta_{1}, \xi_{0} \in \Delta_{0}$. Indeed, otherwise the wall $H_{1}$ does not separate $\Delta_{0}$ from $\Delta$, but separates $\Delta_{1}$ from $\Delta$. Then $H_{1}$ does not separate $\Delta_{m}$ from $\Delta$ either. Thus, as in the proof of Proposition 3.7, we can replace the gallery $\left(\Delta_{0}, \Delta_{1}, \ldots, \Delta_{m}\right)$ with a shorter gallery connecting $\Delta_{0}$ to $\Delta^{\prime}$, contradicting minimality of $m$. Now, clearly,

$$
\eta_{0} \geq \eta_{1}, \quad \eta_{0} \triangleright \eta_{1}
$$

Therefore, by the induction

$$
\eta_{0} \geq \eta_{1} \geq \eta_{m} \Rightarrow v=\eta_{0} \geq \eta_{m}=\mu .
$$

Remark 3.11. The converse to the above lemma is false, for instance, for the root system $A_{2}$. See Figure 3, where $\eta_{0} \geq \eta_{1}$ but $\eta_{0} \not \eta_{1}$.

As a corollary of Lemma 3.10 we obtain: 


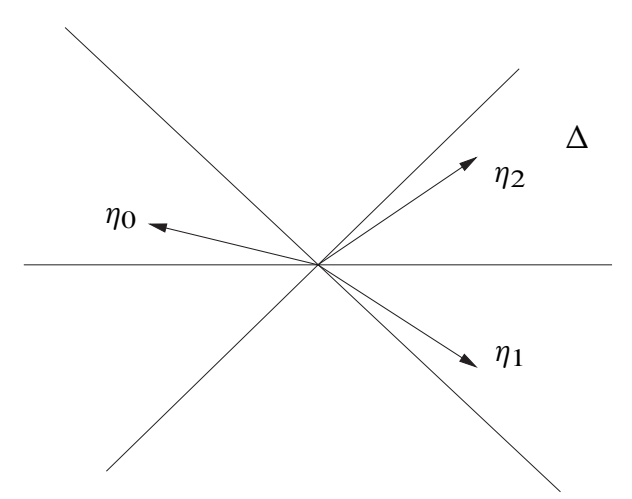

Figure 3. A chain.

Lemma 3.12. Let $v \in V \backslash\{0\}$ and let $\mu$ be the unique vector in $W \cdot v$ which belongs to $\Delta$. Then $v \geq \mu$.

Proof. Clearly, $\mu \triangleright v$. Then the assertion follows from Lemma 3.10.

Definition 3.13 (Maximality condition). We say that a chain $\left(\eta_{0}, \eta_{1}, \ldots, \eta_{m}\right)$ is maximal if it cannot be subdivided into a longer $W$-chain. Equivalently, $\operatorname{dist}\left(\eta_{i}, \eta_{i+1}\right)=1$ for each $i=0, \ldots, m-1$.

Lemma 3.14. Suppose that $v \geq \mu$, and there exists a simple root $\beta$ such that $\tau_{\beta}(v)=\mu$ and $\beta(v)<0$. Then $\operatorname{dist}(v, \mu)=1$.

Proof. Consider a chain from $v$ to $\mu$, i.e., a sequence of vectors $v=v_{0}, v_{1}, \ldots, v_{s}=$ $\mu$ and positive roots $\beta_{1}, \ldots, \beta_{s}$ so that

$$
v_{i}=\tau_{\beta_{i}}\left(v_{i-1}\right) \quad \text { and } \quad \beta_{i}\left(v_{i-1}\right)<0, \quad i=1, \ldots, s .
$$

Then

$$
\mu-v=-\beta(v) \beta^{\vee}
$$

and

$$
\mu-v=\sum_{i=1}^{s}-\beta_{i-1}\left(v_{i}\right) \beta_{i}^{\vee}
$$

Thus

$$
\beta=\sum_{i=1}^{s} \frac{\left\langle v_{i}, \beta_{i}^{\vee}\right\rangle}{\left\langle v, \beta^{\vee}\right\rangle} \beta_{i-1},
$$

i.e., the simple root $\beta$ is a positive linear combination of positive roots. It follows that $s=1$ and $\beta_{1}=\beta$. 


\subsection{Relative chains}

1. Chains relative to a root subsystem. Let $R$ be a root system on $V$ with the set of simple roots $\Phi, W$ be the corresponding Weyl group. Let $\Phi^{\prime} \subset \Phi$ be a subset, $W^{\prime} \subset W$ the corresponding reflection subgroup and $\Delta^{\prime}$ the positive chamber for $W^{\prime}$, defined by the property that all simple roots $\alpha \in \Phi^{\prime}$ are nonnegative on $\Delta^{\prime}$. Thus we will be (frequently) considering $\left(V, W^{\prime},-\Delta^{\prime}\right)$-chains rather than $(V, W,-\Delta)$-chains. In this paper we will be using subgroups $W^{\prime}$ which are stabilizers of points $x$ in ( $\left.A, W_{\text {aff }}\right)$ (where $W=W_{\text {sph }}$ ). Then we will think of a relative $\left(V, W^{\prime}\right)$-chain as a chain in the tangent space $T_{x} A$ of the point $x$.

Lemma 3.15. Given a nonzero vector $\eta \in V$ there exists a $W^{\prime}$-chain $\left(\eta=\eta_{0}, \ldots, \eta_{m}\right)$ with $\eta_{m} \in \Delta^{\prime}$, so that the chain $\left(\eta_{i}\right)$ is maximal with respect to the original root system $R$.

Proof. The proof is by induction on the number $r=r(\eta)$ of simple roots in $\Phi^{\prime}$ which are negative on the vector $\eta$. If $r(\eta)=0$ then $\eta \in \Delta^{\prime}$ and there is nothing to prove. Suppose the assertion holds for all $\eta$ with $r(\eta) \leq k$. Let $\eta$ be such that $r(\eta)=k+1$. Pick a root $\beta \in \Phi^{\prime}$ such that $\beta(\eta)<0$. Then for the vector

$$
\zeta:=\tau_{\beta}(\eta)
$$

we have

$$
\zeta=\eta-\beta(\eta) \beta^{\vee}
$$

Clearly, $\beta(\zeta)>0$. Thus the pair $(\eta, \zeta)$ is a $W^{\prime}$-chain; this chain is maximal as a $W$-chain by Lemma 3.14, since it is defined using a simple reflection in $W$.

For each simple root $\alpha \in \Phi^{\prime} \backslash\{\beta\}$ which is nonnegative on $\eta$

$$
\alpha(\zeta)=\alpha(\eta)-\beta(\eta) \alpha\left(\beta^{\vee}\right) \geq \alpha(\eta) \geq 0 .
$$

Therefore $r(\zeta)<r(\eta)$ and we are done by the induction.

Lemma 3.16. Suppose that $W^{\prime} \subset W$ is a reflection subgroup as above. For any two vectors $\alpha, \delta$ the following are equivalent:

(1) There exists $\beta, \gamma$ so that $\alpha \geq W^{\prime} \beta \sim_{W} \gamma \geq_{W^{\prime}} \delta$.

(2) There exists $\beta$ so that $\alpha \geq_{W^{\prime}} \beta \sim_{W} \delta$.

(3) There exists $\gamma$ so that $\alpha \sim_{W} \gamma \geq_{W^{\prime}} \delta$.

Here $\sim$ is the relation from Definition 2.4 .

Proof. It is clear that (2) $\Longrightarrow$ (1) and (3) $\Longrightarrow$ (1). We will prove that (1) $\Longrightarrow$ (2), since the remaining implication is similar. We have chains

$$
\begin{array}{ll}
\left(\alpha=\eta_{0}, \ldots, \eta_{m}=\beta\right), & \eta_{i}=\tau_{i}\left(\eta_{i-1}\right), i=1, \ldots, m, \\
\left(\gamma=\eta_{0}^{\prime}, \ldots, \eta_{s}^{\prime}=\delta\right), & \eta_{i}^{\prime}=\tau_{i}^{\prime}\left(\eta_{i-1}^{\prime}\right), i=1, \ldots, s .
\end{array}
$$


Then we can extend the first chain to

$$
\left(\alpha=\eta_{0}, \ldots, \eta_{m}=\beta, \tau_{1}^{\prime}(\beta)=\eta_{m+1}, \ldots, \tau_{s}^{\prime}\left(\eta_{m+s-1}\right)=\eta_{m+s}=: \epsilon\right) .
$$

After discarding equal members of this sequence we obtain a chain from $\alpha$ to $\epsilon$. Since $\beta, \gamma$ belong to the same chamber, the vectors $\epsilon$ and $\delta=\tau_{s}^{\prime} \circ \cdots \circ \tau_{1}^{\prime}(\gamma)$ also belong to the same chamber. Therefore we obtain

$$
\alpha \geq \epsilon \sim \delta
$$

Definition 3.17. We will write $\alpha \gtrsim W^{\prime} \delta$ if one of the equivalent conditions in the above lemma holds. We will frequently omit the subscript $W^{\prime}$ in this notation when the choice of the subgroup $W^{\prime}$ is clear or irrelevant.

The reason for using the relation $\sim$ with respect to $W$ rather than its subgroup $W^{\prime}$ (as may seem more natural) is that we will be taking limits under which the subgroup $W^{\prime}$ is increasing (but the limit is still contained in $W$ ). Such limits clearly preserve the relation $\gtrsim_{W^{\prime}}$, but not the relation defined using $\sim_{W^{\prime}}$.

The next corollary immediately follows from Lemma 3.16:

Corollary 3.18. $\alpha \gtrsim \delta \Longleftrightarrow-\delta \gtrsim-\alpha$.

Lemma 3.19. Suppose that $v \gtrsim \mu$ and $\alpha \in \Phi$ is such that $\alpha(v)<0, \alpha(\mu) \geq 0$. Then

$$
\tau_{\alpha}(v) \gtrsim \mu \text {. }
$$

Proof. Let $\lambda$ be such that

$$
v \geq \lambda \sim \mu .
$$

Then $\alpha(\lambda) \geq 0$ and it follows that $\lambda \neq v$, i.e., $v>\lambda$. By applying [L2], Lemma 4.3, we get

$$
\tau_{\alpha}(v) \geq \lambda \sim \mu .
$$

2. Chains relative to positive real numbers ( $a$-chains in the sense of Littelmann). Let $a$ be a positive real number and let $\nu, \mu \in V$ be nonzero vectors in the same $W$-orbit.

Definition 3.20 (P. Littelmann, [L2]). An a-chain for $(\nu, \mu)$ is a chain $\left(\lambda_{0}, \ldots, \lambda_{s}\right)$ which starts at $\nu$, ends at $\mu$ and satisfies the following conditions:

(i) For each $i>0$ we have

$$
t_{i}:=\beta_{i}\left(a \lambda_{i-1}\right) \in \mathbb{Z},
$$

where $\lambda_{i}=\tau_{\beta_{i}}\left(\lambda_{i-1}\right)$ as in Definition 3.1.

(ii) For each $i, \operatorname{dist}\left(\lambda_{i-1}, \lambda_{i}\right)=1$. 
Remark 3.21. Our root system $R$ is the coroot system for the one considered by Littelmann.

Our goal is to give this definition a somewhat more geometric interpretation. In particular, we will see that the concept of an $a$-chain is a special case of the concept of a chain relative to a root subsystem.

The root system $R$ defines an affine Coxeter complex $\left(A, W_{\text {aff }}\right)$ on $A$. Let $x \in$ $P\left(R^{\vee}\right)$ be a special vertex; set $x_{i}:=x+a \lambda_{i}, \tau_{i}:=\tau_{\beta_{i}}, i=0, \ldots, s$. Thus $t_{i}=\beta_{i}\left(x_{i-1}\right)$. Note that $t_{i} \in \mathbb{Z}$ iff $\beta_{i}\left(x_{i-1}\right) \in \mathbb{Z}$.

Proposition 3.22. $\beta_{i}\left(x_{0}\right) \in \mathbb{Z}$ for each $i=1,2, \ldots, s$.

Proof. It suffices to consider the case $x=0$. We have

$$
\begin{aligned}
x_{i-1} & =\tau_{i-1}\left(x_{i-2}\right) \\
& =x_{i-2}-\beta_{i-1}\left(x_{i-2}\right) \beta_{i-1}^{\vee}=x_{i-2}-t_{i-1} \beta_{i-1}^{\vee}=\cdots=x_{0}-\sum_{j=1}^{i-1} t_{j} \beta_{j}^{\vee} .
\end{aligned}
$$

Hence

$$
t_{i}=\beta_{i}\left(x_{i-1}\right)=\beta_{i}\left(x_{0}\right)-\sum_{j=1}^{i-1} t_{j} \beta_{i}\left(\beta_{j}^{\vee}\right) .
$$

Since $t_{i} \in \mathbb{Z}$ and $\beta_{i}\left(\beta_{j}^{\vee}\right) \in \mathbb{Z}$ for all $j$, it follows that $\beta_{i}\left(x_{0}\right) \in \mathbb{Z}$.

We define the integers $k_{i}:=\beta_{i}\left(x_{0}\right)$ and the affine walls

$$
H_{i}:=H_{\beta_{i}, k_{i}}=\left\{v \in V: \beta_{i}(v)=k_{i}\right\} .
$$

The reflection $\sigma_{i}$ in the wall $H_{i}$ belongs to the group $W_{\text {aff }}$, its linear part is the reflection $\tau_{i} \in W_{\text {sph }}, i=1, \ldots, s$.

The argument in the above proof can be easily reversed and hence we get

Corollary 3.23. The integrality condition (i) is equivalent to the assumption that the point $x_{0}$ lies on the intersection of walls $H_{i}$ of the Euclidean Coxeter complex ( $\left.A, W_{\text {aff }}\right)$, where each $H_{i}$ is parallel to the reflection hyperplane of $\tau_{i}$. Equivalently, the $W$-chain $\left(\lambda_{0}, \ldots, \lambda_{s}\right)$ is actually a $W^{\prime}$-chain, where $W^{\prime}=W_{x_{0}}$ is the stabilizer of $x_{0}$ in $W_{\text {aff }}$.

Therefore, identify the vectors $\lambda_{i}$ with vectors in the tangent space $V^{\prime}:=T_{x_{0}}(A)$, let $\Delta^{\prime} \subset V^{\prime}$ denote the Weyl chamber of $W^{\prime}$ which contains the (parallel transport of the) positive chamber $\Delta$. We obtain

Proposition 3.24. Littelmann's definition of an a-chain is equivalent to the conjunction of 
(1) $\left(\lambda_{0}, \ldots, \lambda_{s}\right)$ is a chain in $\left(V^{\prime}, W^{\prime},-\Delta^{\prime}\right)$;

(2) this chain is maximal as a $W$-chain.

Thus the choice of the real number $a$ amounts to choosing a Coxeter subcomplex $\left(V^{\prime}, W^{\prime}\right)$ in $\left(V, W_{\text {sph }}\right)$. The reader will also note the discrepancy between (1) and (2): The chain condition refers to the restricted Coxeter complex $\left(V^{\prime}, W^{\prime}\right)$, while the maximality condition refers to the unrestricted one, $\left(V, W_{\mathrm{sph}}\right)$. This is the key difference between LS paths and Hecke paths.

Remark 3.25. Note that both conditions (1) and (2) are vacuous if $x_{0}$ is a special vertex in the Euclidean Coxeter complex (equivalently, if $a v$ is a coweight): If

$$
\left(\lambda_{0}, \lambda_{1}, \ldots, \lambda_{s}\right)
$$

is a $W^{\prime}$-chain, since $W^{\prime} \cong W_{\text {sph }}$, we can subdivide this chain to get a longer chain

$$
\left(\lambda_{0}=\lambda_{0}^{\prime}, \lambda_{1}^{\prime}, \ldots, \lambda_{m-1}^{\prime}, \lambda_{m}^{\prime}=\lambda_{s}\right)
$$

between $\lambda_{0}$ and $\lambda_{s}$ which satisfies the unit distance condition $\operatorname{dist}\left(\lambda_{i}^{\prime}, \lambda_{i+1}^{\prime}\right)=1$ for all $i$.

3.3. Hecke paths. The goal of this section is to introduce a class of piecewiselinear paths which satisfy a condition similar to Littelmann's definition of LS (and generalized LS) paths. These paths (Hecke paths) play the role in the problem of computing structure constants for spherical Hecke algebras which is analogous to the role that LS paths play in the representation theory of complex semisimple Lie groups.

Let $\left(A, W_{\text {aff }},-\Delta\right)$ be a Euclidean Coxeter complex corresponding to a root system $R$, with fixed negative chamber $-\Delta$. Let $p \in \widetilde{\mathcal{P}}$ be a path equal to the composition

$$
\overline{x_{1} x_{2}} \cup \cdots \cup \overline{x_{n-1} x_{n}} .
$$

For each vertex $x=x_{i}, i=2, \ldots, n-1$, we define the unit tangent vectors $\xi, \eta \in S_{x}$ to the segments $\overline{x_{i} x_{i-1}}, \overline{x_{i} x_{i+1}}$.

Definition 3.26. We say that the path $p$ satisfies the chain condition if for each $x=x_{i}, i=2, \ldots, n-1$, there exists a unit vector $\mu$ so that the following holds:

(1) $-\xi \geq W_{x} \mu$ in the spherical Coxeter complex $\left(S_{x}, W_{x}, \Delta\right)$.

(2) $\mu \sim \eta$ in the (unrestricted) spherical Coxeter complex $\left(S, W_{\text {sph }}\right)$, i.e., for each root $\alpha \in R$ we have

$$
\alpha(\mu) \geq 0 \Longleftrightarrow \alpha(\eta) \geq 0
$$

in other words, for each $t \in[0,1]$ we have

$$
p_{-}^{\prime}(t) \gtrsim W_{p(t)} p_{+}^{\prime}(t) .
$$


Intuitively, at each break point $p(t)$ the path $p$ "turns towards the positive chamber". In what follows we will use the notation $\mathcal{P}_{\text {chain }}$ for the set of all paths $p \in \mathcal{P}$ satisfying the chain condition.

Definition 3.27. A path $p \in \widetilde{\mathcal{P}}$ is called a Hecke path if it is a billiard path which satisfies the chain condition, i.e., for each $t$

$$
p_{-}^{\prime}(t) \geq W_{p(t)} p_{+}^{\prime}(t)
$$

Below is an example of a class of Hecke paths. Suppose that $p \in \mathcal{P}$ and for each $t \in[0,1]$ either $p$ is smooth at $t$ or there exists a reflection $\tau \in W_{p(t)}$ such that the derivative of $\tau$ equals $\tau_{\beta}, \beta \in R^{+}$, and

(1) $d \tau_{p(t)}\left(p_{-}^{\prime}(t)\right)=p_{+}^{\prime}(t)$;

(2) $\beta\left(p_{-}^{\prime}(t)\right)<0, \beta\left(p_{+}^{\prime}(t)\right)>0$.

Then $p$ is a Hecke path with the length of each chain in $\left(S_{p(t)}, W_{p(t)}\right)$ equal to 0 or 1 . See Figure 4.

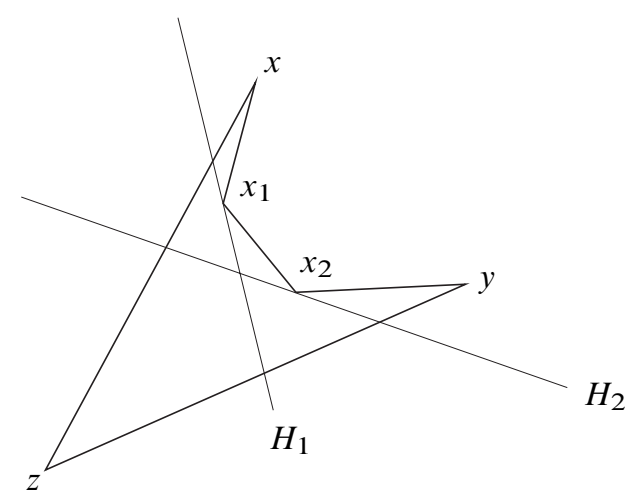

Figure 4. A billiard path satisfying the simple chain condition.

Definition 3.28. We say that $p$ satisfies the simple chain condition if at each break point $x=p(t)$ the chain can be chosen to be simple, i.e., of length 1 .

In what follows we will also need

Definition 3.29. Suppose that $p$ is a path satisfying the chain condition. We call $p$ a generalized Hecke path if each geodesic segment in $p$ (regarded as a vector) is an integer multiple of some $w \varpi$, where $w \in W$ and $\varpi=\varpi_{i}$ is one of the fundamental coweights. 
3.4. A compactness theorem. Pick $\epsilon>0$. We define the subset $\mathcal{P}_{m, \epsilon} \subset \mathcal{P}$ consisting of paths $p$ with

$$
\underline{\text { length }}_{\Delta}(p)=\underline{\lambda}=\left(\lambda_{1}, \ldots, \lambda_{m}\right)
$$

so that for each $i$,

$$
\epsilon \leq\left|\lambda_{i}\right| \leq \epsilon^{-1}
$$

Theorem 3.30. For each $\epsilon>0$ the set $\mathcal{P}_{\text {chain }, m, \epsilon}:=\mathcal{P}_{\text {chain }} \cap \mathcal{P}_{m, \epsilon}$ is compact in $\mathcal{P}_{m, \epsilon}$.

Proof. Suppose that $p \in \mathcal{P}_{\text {chain }}$ is a concatenation of $m$ billiard paths $p_{i}$. Then the number of breaks in the broken geodesic $p_{i}$ is bounded from above by a constant $c$ equal to the length of a maximal chain in the Bruhat order of the finite Weyl group $W_{\mathrm{sph}}$; see Corollary 3.8. This immediately implies that the subset $\mathcal{P}_{\text {chain, }, \epsilon, \epsilon}$ is precompact in $\mathcal{P}$. What has to be proven is that this subset is closed.

Let $\lambda_{i j} \in V$ be vectors of nonzero length so that

$$
p_{i}=\pi_{\lambda_{i 1}} * \pi_{\lambda_{i 2}} * \cdots * \pi_{\lambda_{\text {in }}}
$$

are Hecke paths, $i=1,2, \ldots$ We suppose that

$$
\lim _{i \rightarrow \infty} \lambda_{i j}=0, \quad j=2, \ldots, n-1,
$$

and

$$
\lim _{i \rightarrow \infty} \lambda_{i 1}=\lambda_{1 \infty}, \quad \lim _{i \rightarrow \infty} \lambda_{i n}=\lambda_{\infty, n}
$$

are nonzero vectors. It is clear that

$$
\lim _{i} p_{i}=p_{\infty}:=\pi_{\lambda_{\infty, n}} * \pi_{\lambda_{\infty, n}} .
$$

Lemma 3.31. Under the above conditions the path $p_{\infty}$ is again a Hecke path.

Proof. Let $x:=\lambda_{\infty, n}$. We need to check that the unit vectors $\bar{\lambda}_{\infty, n}, \bar{\lambda}_{\infty, n}$ satisfy

$$
\bar{\lambda}_{\infty, n} \geq_{W_{x}} \bar{\lambda}_{\infty, n} .
$$

Here and below, $W_{x}$ is the stabilizer of $x \in V$ in the Coxeter group $W_{\text {aff }}$.

Let $x_{i j}$ denote the break point of $p_{i}$ which is the concatenation point between $\lambda_{i, j}$ and $\lambda_{i, j+1}$. Then

$$
\bar{\lambda}_{i, j} \geq_{W_{x_{i j}}} \bar{\lambda}_{i, j+1},
$$

$\lim _{i} x_{i j}=x$. If $\sigma_{i j} \in W_{\text {aff }}$ is a reflection fixing $x_{i j}$, then, up to a subsequence,

$$
\sigma_{\infty, j}=\lim _{i} \sigma_{i j} \in W_{\text {aff }}
$$


fixes the point $x$. Therefore it follows from the definition of a chain that

$$
\bar{\lambda}_{\infty, j} \geq_{W_{x}} \bar{\lambda}_{\infty, j+1}
$$

for each $j$. By putting these inequalities together we obtain that

$$
\bar{\lambda}_{\infty, 1} \geq_{W_{x}} \bar{\lambda}_{\infty, n} .
$$

Suppose now that $p_{i}, q_{i}$ are Hecke paths,

$$
p_{i}=\pi_{\lambda_{i 1}} * \pi_{\lambda_{i 2}} * \cdots * \pi_{\lambda_{i n}}, \quad q_{i}=\pi_{\mu_{i 1}} * \pi_{\mu_{i 2}} * \cdots * \pi_{\mu_{i m}},
$$

and the concatenation $r_{i}:=p_{i} * q_{i}$ satisfies the chain condition. We suppose that $r_{\infty}=p_{\infty} * q_{\infty}$ is the limit of the sequence of paths $r_{i}$ and the paths $p_{\infty}, q_{\infty}$ are not constant.

Lemma 3.32. Under the above assumptions, the path $r_{\infty}$ also satisfies the chain condition.

Proof. Lemma 3.31 implies that the paths $p_{\infty}, q_{\infty}$ are Hecke. Therefore it suffices to verify the chain condition at the concatenation point $x=p_{\infty}(1)$. Up to passing to a subsequence, we have

$$
\begin{aligned}
& \lim _{i \rightarrow \infty} \lambda_{i j}=\lambda_{\infty, j}, \quad \lim _{i \rightarrow \infty} \mu_{i j}=\mu_{\infty, j}, \\
& \lim _{i \rightarrow \infty} \bar{\lambda}_{i j}=\bar{\lambda}_{\infty, j}, \quad \lim _{i \rightarrow \infty} \bar{\mu}_{i j}=\bar{\mu}_{\infty, j} .
\end{aligned}
$$

Suppose that

$$
\lim _{i} \lambda_{i j}=0, j=k+1, \ldots, n, \quad \lim _{i} \mu_{i j}=0, j=1, \ldots, l,
$$

and

$$
\lambda_{\infty, k} \neq 0, \quad \mu_{\infty, l+1} \neq 0
$$

By Lemma 3.31,

$$
\bar{\lambda}_{\infty, k} \geq_{W_{x}} \bar{\lambda}_{\infty, n}, \quad \bar{\mu}_{\infty, 1} \geq_{W_{x}} \bar{\mu}_{\infty, l+1} .
$$

On the other hand, it is clear that

$$
\bar{\lambda}_{\infty, n} \gtrsim_{W_{x}} \bar{\mu}_{\infty, 1} .
$$

Therefore, by Lemma 3.16, we get

$$
\bar{\lambda}_{\infty, k} \gtrsim_{W_{x}} \bar{\mu}_{\infty, l+1} .
$$


We can now finish the proof Theorem 3.30. Suppose that $p_{i}=p_{i 1} * \cdots * p_{i m}$ is a sequence of paths in $\mathcal{P}_{\text {chain, } m, \epsilon}$, where each $p_{i j}$ is a billiard path, and $p_{\infty}$ is the limit of this sequence. Each sequence of billiard paths $\left(p_{i j}\right)_{i \in \mathbb{N}}$ converges to a billiard path $p_{\infty, j}$ which is a Hecke path according to Lemma 3.31. Consider now a concatenation point $x$ of the subpaths $p_{\infty}:=p_{\infty, j}, q_{\infty}:=p_{\infty, j+1}$. The paths $p_{\infty}$, $q_{\infty}$ are non-constant by the inequality (3). Therefore we can apply Lemma 3.32 to conclude that the path $p_{\infty}$ satisfies chain condition at the point $x$.

Remark 3.33. It is easy to see that the assumption that the length of each billiard subpath $p_{i j}$ is bounded away from zero is necessary in Theorem 3.30.

\section{Folding}

The key tool for proving the main results of this paper is folding of polygons in a building $X$ into apartments and Weyl chambers. The folding construction replaces a geodesic segment $\tilde{p}$ in $X$ with a piecewise-linear path $p$ in an apartment. This construction was used in [KLM3] to construct various counterexamples. The reader will note that the folding construction used in the present paper is somewhat different from the one in [KLM3].

4.1. Folding via retraction. Suppose that $X$ is a Euclidean or spherical building modeled on the Coxeter complex $(A, W)$, we identify the model apartment $A$ with an apartment $A \subset X$; let $a \subset A$ be an alcove (or a chamber in the spherical case). Recall that the retraction, or folding, to an apartment $f=\operatorname{Fold}_{a, A}: X \rightarrow A$ is defined as follows (see for instance [Rou]):

Given a point $x \in X$ choose an apartment $A_{x}$ containing $x$ and $a$. Then there exists a (unique) isomorphism $\phi: A_{x} \rightarrow A$ fixing $A \cap A_{x}$ pointwise and therefore fixing $a$ as well. We let $f(x):=\phi(x)$. It is easy to see that $f(x)$ does not depend on the choice of $A_{x}$. Observe that $f$ is an isometry on each geodesic $\overline{x y}$, where $y \in a$.

The retraction can be generalized as follows.

Suppose that $X$ is a Euclidean building, $a$ is an alcove with a vertex $v$ (not necessarily special). Let $\Delta \subset A$ denote a Weyl chamber with tip $o$. Choose a dilation $h \in \operatorname{Dil}(A, W)$ which sends $v$ to $o$. Let $\mathbb{P}: A \rightarrow \Delta$ denote the natural projection which sends points $x \in A$ to $W_{\text {sph }} \cdot x \cap \Delta$, where $W_{\text {sph }}$ is the stabilizer of $o$ in $W_{\text {aff }}$.

We define a folding $g=$ Fold $_{v, h, \Delta}: X \rightarrow \Delta$ as the composition

$$
\mathbb{P} \circ h \circ \operatorname{Fold}_{a, A} .
$$

The mapping $g$ will be called a folding into a Weyl chamber. Observe that $g$ (unlike Fold $_{a, A}$ ) does not depend upon the choice of the alcove $a$, therefore it will be denoted 
in what follows $g=$ Fold $_{v, h, \Delta}$. Note that

$$
\operatorname{Fold}_{v, k \circ h, \Delta}=k \circ \operatorname{Fold}_{v, h, \Delta} .
$$

In case when $h=$ id we will abbreviate $\operatorname{Fold}_{v, h, \Delta}$ to $\operatorname{Fold}_{\Delta}$.

Remark 4.1. The folding maps are Lipschitz and differentiable. The restriction of the retraction Fold $_{a, A}$ to each chamber (alcove) is a congruence of two chambers (alcoves).

If $h=\mathrm{id}$ (and thus $v=o$ ) one can describe $f=$ Fold $_{\Delta}$ as follows. Given a point $x \in X$ find an apartment $A_{x}$ through $v, x$ and a Weyl chamber $\Delta_{x} \subset A_{x}$ with tip $v$. Let $\phi: \Delta_{x} \rightarrow \Delta$ be the unique isometry extending to an isomorphism of Coxeter complexes $A_{x} \rightarrow A$. Then $f(x)=\phi(x)$.

Suppose now that $X$ is a Euclidean building, $x \in X$; we give the link $\Sigma_{x}(X)$ structure of an unrestricted spherical building $Y$. Let $R$ denote the corresponding root system. Let $\delta$ be a chamber in $Y$ and $\xi, \mu \in \delta$. Let $f: X \rightarrow \Delta$ be a folding of $X$ to a Weyl chamber. Let $x^{\prime}, \xi^{\prime}, \mu^{\prime}$ denote the images of $x, \xi, \mu$ under $f$ and $d f_{x}$. Then

Lemma 4.2. (1) $d_{\text {ref }}(\xi, \mu)=d_{\text {ref }}\left(\xi^{\prime}, \mu^{\prime}\right)$.

(2) For each $\alpha \in R$,

$$
\alpha\left(\mu^{\prime}\right) \geq 0 \Longleftrightarrow \alpha\left(\xi^{\prime}\right) \geq 0 .
$$

Proof. The restriction $\left.d f\right|_{\delta}$ is an isometry which is the restriction of an isomorphism of spherical apartments. This proves (1). To prove (2) observe that $d f$ sends $\delta$ to a spherical Weyl chamber $\delta^{\prime}$ in $\Sigma_{x^{\prime}} X$.

Let $f$ be a folding of $X$ into an apartment or a chamber.

Lemma 4.3. For each geodesic segment $\overline{x y} \subset X$ its image $f(\overline{x y})$ is a broken geodesic, i.e., it is a concatenation of geodesic segments.

Proof. We give a proof in the case of a folding into an apartment and will leave the other case to the reader. Let $A^{\prime} \subset X$ denote an apartment containing the geodesic segment $\overline{x y}$. Let $a_{1}, \ldots, a_{m}$ denote the alcoves (or chambers in the spherical case) in $A^{\prime}$ covering $\overline{x y}$, set $\overline{x_{i} x_{i+1}}:=\overline{x y} \cap a_{i}$. For each $a_{i}$ there exists an apartment $A_{i}$ containing the alcoves $a$ and $a_{i}$. The restriction of the retraction $f$ to $A_{i}$ is an isometry. It is now clear that the path $f(\overline{x y})$ is a composition of the geodesic paths $f\left(\overline{x_{i} x_{i+1}}\right)$.

We let $x_{i}^{\prime}=f\left(x_{i}\right)$ denote the break points of $f(\overline{x y})$. For each $x_{i}^{\prime}$ let $\xi_{i}^{\prime}, \eta_{i}^{\prime}$ denote the unit tangent vectors in $T_{x_{i}^{\prime}} A$ which are tangent to the segments $\overline{x_{i}^{\prime} x_{i-1}^{\prime}}, \overline{x_{i}^{\prime} x_{i+1}^{\prime}}$ respectively. 
Lemma 4.4. The broken geodesic $f(\overline{x y})$ is a billiard path, i.e., for each break point $x_{i}^{\prime}$ the vectors $\xi^{\prime}:=\xi_{i}^{\prime}, \eta^{\prime}:=\eta_{i}^{\prime}$ satisfy that

$$
\text { there exists } w \in W_{x_{i}^{\prime}}: w\left(\xi^{\prime}\right)=-\eta^{\prime} \text {. }
$$

Proof. We again present a proof only in the case of a folding into an apartment. Let $Y=\Sigma_{x_{i}} X$ denote the spherical building which is the space of directions of $X$ at $x_{i}$. Let $\Delta_{Y}$ denote the Weyl chamber of this building and $\theta: Y \rightarrow \Delta_{Y}$ the canonical projection. The directions $\xi=\xi_{i}$ and $\eta=\eta_{i}$ of the segments $\overline{x_{i} x_{i-1}}, \overline{x_{i} x_{i+1}}$ are antipodal in the building $Y$. Since the folding $f$ is an isomorphism of the apartments $A_{i} \rightarrow A, A_{i+1} \rightarrow A$, and

$$
d f(\eta)=\eta^{\prime}, \quad d f(\xi)=\xi^{\prime},
$$

we see that

$$
\theta(\xi)=\theta\left(\xi^{\prime}\right), \quad \theta(\eta)=\theta\left(\eta^{\prime}\right)
$$

The assertion now follows from Lemma 2.13, part (2).

Lemma 4.5. Suppose that $X$ is a Euclidean building, $f=\operatorname{Fold}_{a, A}: X \rightarrow A$ and $g=$ Fold $_{z, h, \Delta}$ are foldings to an apartment and a chamber respectively. Then for each piecewise-linear path $p$ in $X$ we have:

(1) length $_{\Delta}(f(p))=$ length $_{\Delta}(p)$;

(2) length $\Delta(g(p))=k \cdot$ length $_{\Delta}(p)$, where $k>0$ is the conformal factor of the

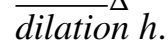

Proof. We will prove the first assertion since the second assertion is similar. It suffices to give a proof in the case when $p$ is a billiard path. Then, analogously to the proof of Lemma 4.4, there exists a representation of $p$ as a composition of geodesic subpaths

$$
p=p_{1} \cup \cdots \cup p_{m}
$$

so that the restriction of $f$ to each $p_{i}$ is a congruence. Therefore

$$
\operatorname{length}_{\Delta}\left(p_{i}\right)=\text { length }_{\Delta}\left(f\left(p_{i}\right)\right)
$$

and hence

$$
\operatorname{length}_{\Delta}(p)=\sum_{i} \operatorname{length}_{\Delta}\left(p_{i}\right)=\text { length }_{\Delta}(f(p)) .
$$

Derivative of the retraction. We assume that $\operatorname{rank}(X) \geq 1$. We identify the model apartment $A$ with an apartment in $X$. Pick $a \subset A$ which is an alcove (in the Euclidean case) or a chamber (in the spherical case). Given a point $x^{\prime} \in X$ choose an apartment $\left(A^{\prime}, W^{\prime}\right)$ through $a$ and $x^{\prime}$ and let $\phi: A \rightarrow A^{\prime}$ denote the inverse to the retraction 
$f=$ Fold $_{a, A}: A^{\prime} \rightarrow A$. Set $x=f\left(x^{\prime}\right)$ and let $W_{x}^{\prime}$ denote the stabilizer of $x^{\prime}$ in $W^{\prime}$. Then the link $Y=\Sigma_{x^{\prime}}(X)$ has a natural structure of a thick spherical building modeled on $\left(S, W_{x}^{\prime}\right)$. It is easy to see that $\left(S, W_{x}^{\prime}\right)$ is independent of the choice of $A^{\prime}$. Observe that if $x^{\prime}$ is antipodal to a regular point $y \in a$ then $x^{\prime}$ is regular itself and therefore $W_{x}^{\prime}=\{1\}$. We next define a chamber $s \subset S$ :

Given a regular point $y \in a \backslash\left\{x^{\prime}\right\}$ and a geodesic segment $\overline{x^{\prime} y}$, let $\zeta=\zeta(y)$ denote the unit tangent vector to $\overline{x^{\prime} y}$ at $x^{\prime}$. Then the set

$$
\{\zeta(y): y \text { is a regular point in } a\}
$$

is contained in a unique spherical chamber $s \subset S$. (If $x^{\prime}$ is antipodal to some $y \in \operatorname{int}(a)$ then $s=S$.)

Set $f^{\prime}:=\phi \circ f=$ Fold $_{a, A^{\prime}}$.

Lemma 4.6. The derivative $d_{x^{\prime}} f^{\prime}: Y \rightarrow S$ equals Fold $_{s, S}$.

Proof. Given $\eta \in Y$, find an alcove (or a spherical chamber) $c$ so that $\eta \in \Sigma_{x^{\prime}} c$. Then there exists an apartment $A_{\eta} \subset X$ containing both $a$ and $c$. Let $S_{\eta}$ denote the unit tangent sphere of $A_{\eta}$ at $x^{\prime}$. Then $\eta \in S_{\eta}$ and $s \subset S_{\eta}$. Now it is clear from the definition that

$$
d f^{\prime}(\eta)=\operatorname{Fold}_{s, S}(\eta)
$$

since both maps send $S_{\eta}$ to $S$ and fix $s$ pointwise.

Folding of polygons. Suppose now that $X$ is a building and $\widetilde{P}=\left[\tilde{z}, \tilde{x}_{1}, \ldots, \tilde{x}_{n}\right]$ is a geodesic polygon in $X$. Pick an apartment $A \subset X$ which contains $\tilde{\tilde{z}} \tilde{x}_{1}$ and an alcove $a \subset A$ which contains $\tilde{z}$. Let $\Delta \subset A$ denote a Weyl chamber (in case $X$ is Euclidean) with tip $o$. Let $f$ be a folding of $X$ of the form

$$
f=\text { Fold }_{a, A}
$$

or

$$
f=\operatorname{Fold}_{\tilde{z}, h, \Delta},
$$

where $h$ is a dilation sending $\tilde{z}$ to $o$. We will then apply $f$ to $\widetilde{P}$ to obtain a folded polygon $P:=f(\widetilde{P})$ in $A$ or $\Delta$ respectively.

Observe that the restriction of $f$ to the edges $\overline{\tilde{z}} \tilde{x}_{1}$ and $\overline{\tilde{x}_{n} \tilde{z}}$ of $P$ is an isometry or a similarity. The restriction of $f$ to the path

$$
\tilde{p}=\overline{\tilde{x}_{1} \tilde{x}_{2}} \cup \cdots \cup \overline{\tilde{x}_{n-1} \tilde{x}_{n}}
$$

preserves the type of the unit tangent vectors; cf. Lemma 4.5. We will be using foldings into apartments and chambers to transform geodesic polygons in $X$ into folded polygons. 
In the special case when $\widetilde{P}=T$ is a triangle (and thus $n=2$ ), the folded triangle $P=f(T)$ has two geodesic sides $\overline{z w_{1}}:=f\left(\overline{\tilde{z}} \tilde{x}_{1}\right), \overline{x_{2} z}:=f\left(\overline{\tilde{x}_{2} \tilde{z}}\right)$ and one broken side $p:=f\left(\overline{\tilde{x}_{1} \tilde{x}_{2}}\right)$, so we will think of $f(T)$ as a broken triangle.

The next proposition relates folding into a Weyl chamber with the concept of folding of polygons used in [KLM3]. Let $P=\left[o, x_{1}, x_{2}, \ldots, x_{n}\right]$ be a polygon in $\Delta$. Triangulate $P$ from the vertex $o$ into geodesic triangles $T_{i}=\left[x_{i}, x_{i+1}, o\right]$. Suppose that $\widetilde{P} \subset X$ is a geodesic polygon

$$
\widetilde{P}=\left[o, \tilde{x}_{1}, \tilde{x}_{2}, \ldots, \tilde{x}_{n}\right], \quad \tilde{x}_{1}=x_{1},
$$

triangulated into geodesic triangles $\widetilde{T}_{i}=\left[\tilde{x}_{i}, \tilde{x}_{i+1}, o\right]$, where each $\widetilde{T}_{i}$ is contained in an apartment $A_{i}$. Assume that for each $i$ there exists a congruence

$$
\phi_{i}: \widetilde{T}_{i} \rightarrow T_{i}
$$

i.e., an isometry sending $\tilde{x}_{j}$ to $x_{j}(j=i, i+1)$ which extends to an isomorphism of Coxeter complexes $\phi_{i}: A_{i} \rightarrow A$.

Proposition 4.7. Under the above assumptions, for each $i$, Fold $_{\Delta}\left|\widetilde{T}_{i}=\phi_{i}\right| \widetilde{T}_{i}$.

Proof. Let $\Delta_{i} \subset A_{i}$ denote the preimage of $\Delta$ under $\phi_{i}$. Then each $\Delta_{i}$ is a Weyl chamber, hence $\phi_{i} \mid \Delta_{i}=$ Fold $_{\Delta} \mid \Delta_{i}$, by the alternative description of Fold given earlier in this section.

The following lemma shows that unfolding of polygons is a local problem. Suppose that $T=\left[o, x_{1}, \ldots, x_{n}\right] \subset A$ is a geodesic polygon so that $x_{i} \neq o$ for each $i$. For each $i=2, \ldots, n-1$ we define the unit vectors

$$
\xi_{i}, \eta_{i}, \zeta_{i} \in \Sigma_{x_{i}} A
$$

which are tangent to the segments $\overline{x_{i} x_{i-1}}, \overline{x_{i} x_{i+1}}, \overline{x_{i} o}$. Define thick spherical buildings $Y_{i}:=\Sigma_{x_{i}}(X)$. By combining the above proposition with [KLM3], condition 7.5, we obtain

Lemma 4.8. The polygon $T$ can be unfolded in $X$ to a geodesic triangle $\widetilde{T}$ whose vertices project to o, $x_{1}, x_{n}$ if and only if for each $i=2, \ldots, n-1$, there exists a triangle $\left[\tilde{\xi}_{i}, \tilde{\zeta}_{i}, \tilde{\eta}_{i}\right] \subset Y_{i}$ so that

$$
\begin{aligned}
d_{\text {ref }}\left(\tilde{\xi}_{i}, \tilde{\zeta}_{i}\right) & =d_{\text {ref }}\left(\xi_{i}, \zeta_{i}\right), \\
d_{\text {ref }}\left(\tilde{\eta}_{i}, \tilde{\zeta}_{i}\right) & =d_{\text {ref }}\left(\eta_{i}, \zeta_{i}\right), \\
d\left(\tilde{\xi}_{i}, \tilde{\eta}_{i}\right) & =\pi .
\end{aligned}
$$

We will eventually obtain a characterization of the broken triangles in $\Delta$ which are foldings of geodesic triangles in $X$ as billiard triangles satisfying the chain condition; see Section 6. The goal of the next section is to give a necessary condition for a broken triangle to be unfolded; we also give a partial converse to this result. 
4.2. Converting folded triangles in spherical buildings into chains. Suppose that $X$ is a (thick) spherical or Euclidean building modeled on $(A, W)$. Consider a triangle $\widetilde{T}=[x, y, z] \subset X$ with

$$
\beta:=d_{\text {ref }}(x, y), \quad \gamma:=d_{\text {ref }}(y, z) .
$$

Assume that $A$ is embedded in $X$ so that it contains $x$ and $z$. Let $a \subset A$ be a spherical chamber or a Euclidean alcove containing $z$. In the spherical case we regard $a$ as the negative chamber in $A$, let $\Delta$ denote the positive chamber $-a$. We have the retraction $f:=$ Fold $_{a, A}: X \rightarrow A$.

Theorem 4.9. There exists a $(A, W, a)$-chain $\left(y_{0}, \ldots, y_{m}\right)$ such that $y_{m}=f(y)$, $d_{\mathrm{ref}}\left(x, y_{0}\right)=\beta, d_{\mathrm{ref}}\left(y_{m}, z\right)=\gamma$. (In the case when $X$ is a Euclidean building the above chain is an affine chain.) See Figure 5.

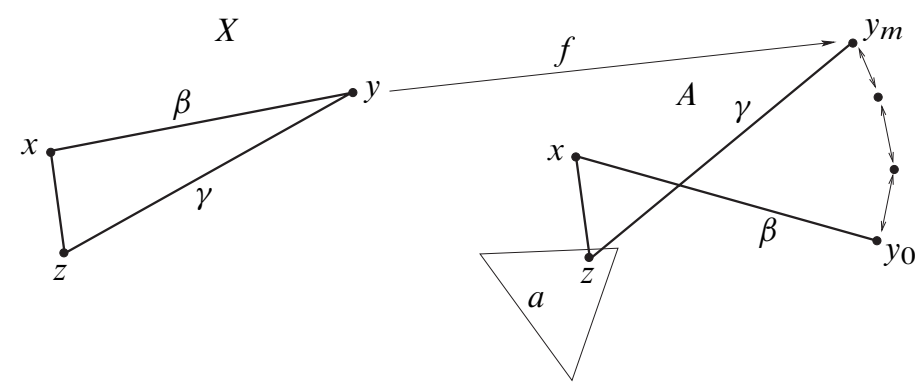

Figure 5. Converting geodesic triangle to a chain.

Proof. We prove the assertion for the spherical buildings as the Euclidean case is completely analogous. (This is also the only case when this theorem is used in the present paper.)

Our proof is by induction on the rank of the building. Consider first the case when $\operatorname{rank}(X)=0$ (i.e., $A=S^{0}$ is the 2-point set). If $y$ and $x$ are both distinct from $z$, then $f(y) \neq z$. This implies that $f(y)=x$ and we take

$$
y_{0}:=z, \quad y_{1}:=y, \quad m=1 .
$$

In the remaining cases we will use the chain $y_{0}=f(y)=y_{m}$.

Suppose now that $\operatorname{rank}(X)=r \geq 1$ and the assertion holds for all (spherical) buildings of rank $r-1$, let us prove it for buildings of rank $r$.

We let $\tilde{p}:[0, c] \rightarrow \overline{x y}$ denote the unit speed parametrization of $\overline{x y}$ and set $p:=$ $f(\tilde{p})$. We assume for now that $z \notin \overline{x y}$. 
As in the proof of Lemma 4.3, we "triangulate" the geodesic triangle $\widetilde{T}$ into geodesic triangles $\widetilde{T}_{i}:=\left[z, \tilde{x}_{i}, \tilde{x}_{i+1}\right]$, where the points $\tilde{x}_{i}=\tilde{p}\left(t_{i}\right), i=1, \ldots, n$, are chosen so that each triangle $\widetilde{T}_{i}$ is contained in an apartment $A_{i} \subset X$ and the map $f$ restricts to an isometry $f: \widetilde{T}_{i} \rightarrow f\left(\widetilde{T}_{i}\right) \subset A$. Here $\tilde{x}_{0}:=x, \tilde{x}_{n+1}=y$. Observe that each side of $\widetilde{T}_{i}$ has positive length.

Let $\widetilde{S}_{i}:=\Sigma_{\tilde{x}_{i}}\left(A_{i}\right)$ denote the unit tangent sphere at $\tilde{x}_{i}$. Define $\tilde{s}_{i}$ to be the (unique) chamber in $\widetilde{S}_{i}$ containing all the directions of the geodesic segments from $\tilde{x}_{i}$ to the interior of $a$. This determines the positive chamber $\widetilde{\Delta}_{i}=-\tilde{s}_{i} \subset S_{i}$.

Set

$$
\tilde{\eta}_{i}:=\tilde{p}^{\prime}\left(t_{i}\right), \quad \tilde{\xi}_{i}:=-\eta_{i},
$$

and let $\tilde{\zeta}_{i} \in \tilde{S}_{i}$ denote the unit tangent vector to $\overline{\tilde{x}_{i} z}$.

Now, applying the retraction $f$ to all this data, we obtain:

(1) The folded triangle $T=f(\widetilde{T})$ which has two geodesic sides $\overline{z x}, \overline{z y_{m}}$ (where $\left.y_{m}=f(y)\right)$, and the broken side represented by the path $p=f(\tilde{p})$. In particular, $d_{\text {ref }}\left(y_{m}, z\right)=d_{\text {ref }}(y, z)$ (as required by the theorem).

(2) The vertices $x_{i}=p\left(t_{i}\right)=f\left(\tilde{x}_{i}\right)$ of the broken geodesic $p$.

(3) Unit tangent vectors $\xi_{i}=d f\left(\tilde{\xi}_{i}\right), \eta_{i}=d f\left(\tilde{\eta}_{i}\right), \zeta_{i}=d f\left(\tilde{\zeta}_{i}\right)$ in $\Sigma_{x} A$. These vectors are tangent to the segments $\overline{x_{i} x_{i-1}}, \overline{x_{i} x_{i+1}}, \overline{x_{i} z}$ respectively.

(4) The positive chamber $\Delta_{i}=d f\left(\widetilde{\Delta}_{i}\right)$ and the negative chamber $s_{i}=d f\left(\tilde{s}_{i}\right)$ in the spherical Coxeter complex $\left(S_{i}=\Sigma_{x_{i}}(A), W_{i}=W_{x_{i}}\right)$. The negative chamber contains the directions tangent to the geodesic segments from $x_{i}$ to the chamber $a \subset A$.

Our goal is to convert the broken side $p$ of $T$ into a chain in $A$ by "unbending" the broken geodesic $p$ to a geodesic segment in $A$. See Figure 6 .

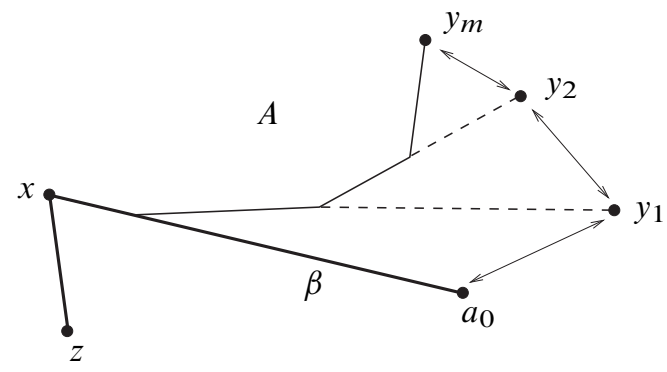

Figure 6. Forming a chain by unbending.

Lemma 4.10. The path $p$ satisfies the following:

(1) The metric lengths of $p$ and $\tilde{p}=\overline{x y}$ are the same. 
(2) $p^{\prime}(0)=\tilde{p}^{\prime}(0)$.

(3) At each break point $x_{i}$ there exists an $\left(S_{i}, W_{i}, s_{i}\right)$-chain from $-\xi_{i}$ to $\eta_{i}$.

Proof. The first two assertions are clear from the construction. Let us prove the last statement. For each $i$ and the point $\tilde{v}=\tilde{x}_{i}$ we have the spherical building $Y:=\Sigma_{\tilde{v}}(X)$ which has rank $r-1$. This building contains the antipodal points

$$
\tilde{\xi}_{i}, \quad \tilde{\eta}_{i}
$$

and the point $\tilde{\zeta}_{i}$. We form the geodesic triangle $\tau=\left[\tilde{\xi}_{i}, \tilde{\eta}_{i}, \tilde{\zeta}_{i}\right] \subset Y$, where we use an arbitrary shortest geodesic in $Y$ to connect $\xi_{i}$ to $\eta_{i}$. Therefore $\xi_{i}, \eta_{i}, \zeta_{i}$ are vertices of the broken geodesic triangle $d f(\tau) \subset S_{i}$.

As in Lemma 4.6, we use the isomorphism $S_{i} \rightarrow \widetilde{S}_{i}$ (sending $s_{i}$ to $\tilde{s}_{i}$ ) to identify these apartments. Under this identification, $d f: Y \rightarrow S_{i}$ is the retraction Fold $_{s_{i}}, S_{i}$ of $Y$ to the apartment $S_{i}$. Thus $d f(\tau)$ is a folded triangle in $S_{i}$.

Hence, by the (rank) induction hypothesis, for each $i$ there exists a chain

$$
\left(-\xi_{i}, \ldots, \eta_{i}\right)
$$

in the spherical Coxeter complex $\left(S_{i}, W_{i}, s_{i}\right)$.

Lemma 4.11. For each path $p:[0, c] \rightarrow$ A satisfying the conclusion of Lemma 4.10, there exists a point $y^{\prime} \in A$ such that

$$
d_{\text {ref }}\left(x, y^{\prime}\right)=\beta \quad \text { and } \quad y^{\prime} \geq p(c)
$$

in $(A, W,-\Delta)$.

Proof. We use the second induction, on the number $n$ of vertices in the broken geodesic $p$. Set $u:=p(c)$.

The metric length of the path $p$ equals the metric length of the path $\tilde{p}=\overline{x y}$, the tangent directions of these paths at $x$ are the same. Therefore, if $n=0$ (and hence the path $p$ is geodesic) there is nothing to prove, one can simply take $y^{\prime}=u$.

Assume that the assertion holds for all $n \leq N-1$, let us prove it for $N$. We treat the path $p$ as the composition

$$
p \mid\left[0, t_{N}\right] \cup \overline{x_{N} u} .
$$

Our goal is to replace the geodesic subpath $\overline{x_{N} u}$ with a geodesic path $w\left(\overline{x_{N} u}\right)$, where $w \in W$ is fixing $x_{N}$, so that:

(1) $\overline{x_{N-1} x_{N}} \cup w\left(\overline{x_{N} u}\right)$ is a geodesic segment.

(2) There exists an $(A, W,-\Delta)$-chain between $w(u)$ and $u$. 
Then we would be done by the induction on $n$. Indeed, the new path

$$
p \mid\left[0, t_{N}\right] \cup w\left(\overline{x_{N} u}\right)
$$

has one less break point and still satisfies the conclusion of Lemma 4.10. Thus, by the induction hypothesis, there exists $y^{\prime} \in A$ so that

$$
\begin{gathered}
y^{\prime} \geq w(u) \geq u \Longrightarrow y^{\prime} \geq u, \\
d_{\text {ref }}\left(x, y^{\prime}\right)=d_{\text {ref }}(x, y) .
\end{gathered}
$$

Construction of $w$. Recall that there exists an $\left(S_{N}, W_{N},-\Delta_{N}\right)$-chain

$$
\left(-\xi_{N}=v_{0}, \ldots, v_{k}=\eta_{N}\right),
$$

hence we have a sequence of reflections $r_{1}, \ldots, r_{k} \in W_{N}$ (fixing walls $H_{i} \subset S_{N}$, $i=1, \ldots, k)$ so that

$$
r_{i}\left(v_{i-1}\right)=v_{i}, \quad i=1, \ldots, k,
$$

and each wall $H_{i}$ separates $v_{i}$ from the negative chamber $s_{N}$. We extend each reflection $r_{i}$ from $S_{N}$ to a reflection $r_{i}$ in $A$, and each $H_{i}$ to a wall $H_{i}$ in $A$.

We therefore define the following points in $A$ :

$$
y_{k}:=u, \quad y_{k-1}:=r_{k}\left(y_{k}\right), \quad y_{k-2}:=r_{k-1}\left(y_{k-1}\right), \ldots, y_{0}:=r_{1}\left(y_{1}\right) .
$$

Note that the directions $v_{i}$ are tangent to the segments $\overline{x_{N} y_{i}}$. Thus for each $i$, the wall $H_{i}$ separates the point $y_{i}$ from the negative chamber $a \subset A$ and the sequence

$$
\left(y_{0}, \ldots, y_{k}=u\right)
$$

forms a chain. We set $w=r_{1} \circ \cdots \circ r_{k}$. The vector $v_{0}$ is antipodal to $-\xi_{N}$, hence the path

$$
p \mid\left[0, T_{N}\right] \cup w\left(\overline{x_{N} u}\right)
$$

is geodesic at the point $x_{N}=p\left(T_{N}\right)$.

This concludes the proof of Theorem 4.9 in the case when $z \notin \overline{x y}$.

We now consider the special case when the above proof has to be modified: The triangle $T$ is degenerate, i.e., $z \in \overline{x y}$, but the alcove $a$ is such that $y \notin A$. Thus the folding Fold $a_{a, A}$ is not an isometry on $T$. Then the tangent direction $\tilde{\zeta}_{i}$ is not defined when $x_{i}=z$. Note that $x_{i}=z$ then is the only break point in the broken side of $T^{\prime}$.

In this case we replace the vertex $z$ with an arbitrary point $z^{\prime}$ in the interior of $a$ and repeat the above arguments. The chains constructed in the process will be independent of the choice of $z^{\prime}$ and thus, after taking the limit $z^{\prime} \rightarrow z$, we obtain a chain as required by the assertion of the theorem. 
Corollary 4.12 (Cf. [KLM3], Theorem 8.2, part 4). Suppose that $X$ is a Euclidean building. Assume that $\alpha:=d_{\Delta}(z, x), \beta:=d_{\Delta}(x, y), \gamma:=d_{\Delta}(y, z)$ are in $P\left(R^{\vee}\right)$ and $x, y, z$ are special vertices of $X$. Then

$$
\alpha+\beta+\gamma \in Q\left(R^{\vee}\right) .
$$

Proof. Let $\left(y_{0}, \ldots, y_{m}\right)$ be an affine chain given by Theorem 4.9. We regard the point $x$ as the origin $o$ in $A$; thus we will view $z, y_{0}, y_{m}$ as vectors in $V$. Then, according to Lemma 2.6,

$$
y_{m}-y_{0} \in Q\left(R^{\vee}\right) .
$$

Consider the vectors $\beta^{\prime}:=y_{0}-x, \gamma^{\prime}:=z-y_{m}, \alpha^{\prime}:=x-z$ in $P\left(R^{\vee}\right)$. By the definition of $\Delta$-length,

$$
\alpha^{\prime} \in W_{\mathrm{sph}} \alpha, \quad \beta^{\prime} \in W_{\mathrm{sph}} \beta, \quad \gamma^{\prime} \in W_{\mathrm{sph}} \gamma .
$$

Therefore, by applying Lemma 2.6 again we see that the differences

$$
\alpha-\alpha^{\prime}, \quad \beta-\beta^{\prime}, \quad \gamma-\gamma^{\prime}
$$

all belong to $Q\left(R^{\vee}\right)$. Since

$$
\alpha^{\prime}+\beta^{\prime}+\gamma^{\prime}=y_{0}-y_{m} \in Q\left(R^{\vee}\right),
$$

the assertion of lemma follows.

The following simple proposition establishes a partial converse to Theorem 4.9:

Proposition 4.13. Suppose that $X$ is a thick spherical building and, as before, the point $z$ belongs to a negative chamber $a=-\Delta$. Then, for each simple chain $\left(y_{0}, y_{1}\right)$ such that $d_{\mathrm{ref}}\left(x, y_{0}\right)=\pi, d_{\mathrm{ref}}\left(z, y_{1}\right)=\beta$, there exists a point $y \in X$ so that

$$
d_{\mathrm{ref}}(y, z)=\gamma \quad \text { and } \quad d_{\mathrm{ref}}(x, y)=\pi .
$$

Remark 4.14. Recall that $d_{\text {ref }}(x, y)=\pi$ means that the points $x$ and $y$ are antipodal.

Proof. Let $\tau\left(y_{0}\right)=y_{1}$ where $\tau$ is a reflection in a wall $H \subset A$ as in the definition of a chain. Let $A=A^{-} \cup A^{+}$be the union of half-apartments, where $A^{-}$is bounded by $H$ and contains $a$. By the definition of a chain, $y_{1} \in A^{-}, y_{0} \in A^{+}$and hence the antipodal point $x=-y_{0}$ belongs to $A^{+}$.

Since $X$ is thick, there exists a half-apartment $B^{-} \subset X$ which intersects $A$ along $H$. Define the apartment $B:=A^{-} \cup B^{-}$; then there exists an isomorphism of Coxeter complexes

$$
\phi: A \rightarrow B, \quad \phi \mid A^{-}=\mathrm{id} .
$$


We set $y:=\phi\left(y_{0}\right)$.

Since $\phi$ is an isomorphism of Coxeter complexes which fixes $z$, it preserves the refined distance to the point $z$ and hence

$$
d_{\text {ref }}(y, z)=d_{\text {ref }}\left(y_{1}, z\right)=\gamma .
$$

The union $C:=A^{+} \cup B^{-}$is also an apartment in $X$. Then there exists an isomorphism $\psi: B^{-} \rightarrow A^{-}$so that

$$
\psi \circ \phi\left|A^{+}=\tau\right| A^{+} .
$$

The isomorphism $\psi$ extends to an isomorphism $\rho: C \rightarrow A$ fixing $A^{+}$pointwise and hence fixing the point $x$. Therefore

$$
d_{\text {ref }}(x, y)=d_{\text {ref }}\left(x, y_{0}\right)=\pi .
$$

4.3. Folding polygons in Euclidean buildings. Our next goal is to show that each folding transforms certain piecewise-linear paths in Euclidean buildings to paths satisfying the chain condition.

Suppose that $X$ is a Euclidean building with model apartment $\left(A, W_{\text {aff }}\right)$, $\Delta \subset A$ is the positive Weyl chamber with the tip $o$. Consider a piecewise-linear path $\tilde{p}:[0, c] \rightarrow \tilde{A}$, which is parameterized with the unit speed, where $\tilde{A} \subset X$ is an apartment. We assume that

$$
\tilde{p}_{-}^{\prime}(t) \sim_{W_{\text {sph }}} \tilde{p}_{+}^{\prime}(t)
$$

for each $t \in[0, c]$; for instance, $\tilde{p}$ could be a geodesic path.

Thus the path $\tilde{p}$ trivially satisfies the chain condition. Let $g: X \rightarrow \Delta$ be a folding into $\Delta, g=$ Fold $_{z, h, \Delta}$ for a certain $z \in A$ and $h$. Recall that the folding $g$ is the composition of three maps:

$$
g=\mathbb{P}_{\Delta} \circ h \circ f, \quad f=\operatorname{Fold}_{a, A},
$$

where $a$ is an alcove in $A$ containing $z, h \in \operatorname{Dil}\left(A, W_{\text {aff }}\right)$ is a dilation sending $z$ to the point $o$. Consider the structure of a Coxeter complex on $A$ given by the pull-back

$$
h^{*}\left(A, W_{\text {aff }}\right) .
$$

We thus get a new (typically non-thick) building structure for $X$, the one modeled on $h^{*}\left(A, W_{\text {aff }}\right)$.

Definition 4.15. We say that a path $\tilde{p}$ is generic if it is disjoint from $z$ and from the codimension 2 skeleton of $X$ and the break points of $\tilde{p}$ are disjoint from the codimension 1 skeleton of $X$, where $X$ is regarded as a building modeled on $h^{*}\left(A, W_{\text {aff }}\right)$.

The main result of this section is 
Theorem 4.16. The folded path $p=g(\tilde{p})$ satisfies the chain condition.

Proof. The proof of this theorem is mostly similar (except for the projection $\mathbb{P}$ which causes extra complications) to the proof of Theorem 4.9 in the previous section. We will prove Theorem 4.16 in two steps: We first establish it for the paths $\tilde{p}$ which are generic. Then we use the compactness theorem to prove it in general.

Proposition 4.17. The conclusion of Theorem 4.16 holds for generic paths $\tilde{p}$.

Proof. If a point $\tilde{x}=\tilde{p}(t)$ is a regular point of $X$, then

$$
d g_{\tilde{x}}: \Sigma_{\tilde{x}}(X) \rightarrow \Sigma_{x}(A), \quad x=g(\tilde{x}),
$$

is an isometry. Thus the path $p$ trivially satisfies the chain condition at the point $x$.

Therefore we assume that $\tilde{x}$ is a singular point of $X$. Since $\tilde{p}$ is assumed to be generic, this point lies on exactly one wall of $X$; moreover, $\tilde{p}$ is geodesic near $\tilde{x}$.

We first analyze what happens to the germ of $\tilde{p}$ at $\tilde{x}$ under the retraction $f$. We suppose that the restriction of $f$ to the germ $(\tilde{p}, \tilde{x})$ is not an isometry (otherwise there is nothing to discuss). Let $\tilde{\zeta} \in \Sigma_{\tilde{x}}(X)$ denote the tangent to the geodesic segment $\overline{\tilde{x} z}$. Let $\eta \in \Sigma_{\tilde{x}}(X \cap \tilde{A})$ be the tangent vector $p^{\prime}(t), \tilde{\xi}:=-\tilde{\eta}$ (this vector is also tangent to the path $p)$. Set $\beta:=d_{\text {ref }}(\tilde{\zeta}, \tilde{\eta})$.

We obtain the triangle $\tau=[\tilde{\xi}, \tilde{\eta}, \tilde{\zeta}]$ in $\Sigma_{\tilde{x}}(X)$. The derivative of the retraction $f$ at $\tilde{x}$ is a retraction of the spherical building $\Sigma_{\tilde{x}}(X)$ into its apartment $S$, after identification of $S$ with the sphere $S_{x^{\prime}}(A), x^{\prime}:=f(\tilde{x})$ (see Lemma 4.6). Define the following elements of $S$ :

$$
\eta^{\prime}:=d f_{\tilde{x}}(\tilde{\eta}), \quad \xi^{\prime}:=d f_{\tilde{x}}(\tilde{\xi}), \quad \zeta^{\prime}:=d f_{\tilde{x}}(\tilde{\zeta}) .
$$

Therefore, according to Theorem 4.9, the folded triangle $\tau^{\prime}=d f_{x}(\tau) \subset S$ yields an $\left(S, W_{x^{\prime}},-\Delta_{x^{\prime}}\right)$-chain ${ }^{2}$

$$
\left(\mu^{\prime}=-\xi^{\prime}, \eta^{\prime}\right)
$$

such that $d_{\text {ref }}\left(\zeta, \eta^{\prime}\right)=d_{\text {ref }}(\tilde{\zeta}, \tilde{\eta})=\beta$.

Here $-\Delta_{x^{\prime}}$ is a chamber in $\left(S, W_{x^{\prime}}\right)$ which contains the unit tangent vector to the segment $\overline{x^{\prime} z^{\prime}}$ where $z^{\prime} \in a$ is a regular point.

Remark 4.18. Note that our assumptions on $\tilde{p}$ imply that $\left(S, W_{x^{\prime}}\right)$ has a unique wall. If the corresponding wall in $A$ does not pass through $z$, then the negative chamber $-\Delta_{x^{\prime}}$ in $\left(S, W_{x^{\prime}}\right)$ is uniquely determined by the condition that it contains the direction tangent to $\overline{x^{\prime} z}$.

\footnotetext{
${ }^{2}$ Which is necessarily simple since $\tilde{p}$ is assumed generic.
} 
Consider now the effect of the rest of the folding $g$ on the path $\tilde{p}$ at $\tilde{x}$. Let $x:=g(\tilde{x})$. We identify $S$ with the unit tangent sphere at the point $x$.

The dilation $h$ clearly preserves the chain condition at $x^{\prime}$ (since it acts trivially on the unit tangent sphere). The restriction of the projection $\mathbb{P}=\mathbb{P}_{\Delta}$ to the germ of $h f(\tilde{p})$ at $h f(\tilde{x})$ is necessarily an isometry (since $\tilde{p}$ is generic), hence it is given

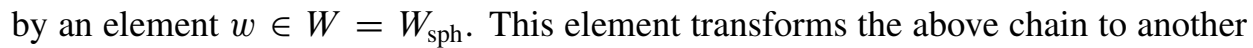
$\left(S, W_{x},-\Delta_{x}\right)$-chain, where

$$
\Delta_{x}:=d(w \circ h)\left(\Delta_{x^{\prime}}\right)
$$

What is left to verify is that the positive chamber $\Delta_{x}$ in this complex contains a translate of the positive chamber $\Delta$. In case when $x$ belongs to the interior of $\Delta$, the segment $\overline{O x}$ is not contained in any wall and thus the negative chamber $-\Delta_{x}$ has to contain the initial direction of the segment $\overline{x z}$ (see the remark above). However this initial direction belongs to $-\Delta$ and thus $\Delta_{x}$ contains $\Delta$.

Consider the exceptional case when $x$ is on the boundary of $\Delta$. It then belongs to a unique wall $H$ in the Coxeter complex $\left(A, W_{\text {aff }}\right)$ and this wall passes through the origin $o$. Rather than trying to use Theorem 4.9 to verify the chain condition at $x$, we give a direct argument. Let $\eta, \xi$ be the unit vectors which are the images of $\eta^{\prime}, \xi^{\prime}$ under

$$
d(w \circ h): \Sigma_{x^{\prime}}(A) \rightarrow \Sigma_{x}(A) .
$$

Since the path $p$ is entirely contained in $\Delta$, the vector $p_{-}^{\prime}(t)$ points outside of $\Delta$ and the vector $p_{+}^{\prime}(t)$ points inside. The reflection $\sigma$ in the wall $H$ sends the vector $-\xi=p_{-}^{\prime}(t)$ the vector $\eta=p_{+}^{\prime}(t)$. It is then clear that the (simple) chain condition is satisfied at the point $x$.

Lastly, we consider the points $\tilde{x}=\tilde{p}(t)$ for which $f$ is an isometry on the germ of $\tilde{p}$ at $\tilde{x}$. The point $x=g(\tilde{x})$ belongs to a face of $\Delta$ contained in a wall $H$, and this is the only wall of ( $\left.A, W_{\text {aff }}\right)$ which passes through $x$. Then, necessarily, the germ of the path $h f(\tilde{p})$ at $h f(\tilde{x})$ is a geodesic. We now simply repeat the arguments of the exceptional case in the above proof (see also the proof of Proposition 4.19) to see that $p$ satisfies the chain condition at $x$.

We are now ready to prove Theorem 4.16 for arbitrary paths $\tilde{p}$. We will do so by approximating the path $\tilde{p}$ via generic paths. Let $\lambda$ be an arbitrary vector in $\tilde{A}$. We let $\tilde{q}_{\lambda}:=\tilde{p}+\lambda$ denote the translation of the path $\tilde{p}$ by the vector $\lambda$. It is clear, from the dimension count, that for an open and dense set of vectors $\lambda$, the path $\tilde{q}_{\lambda}$ is generic.

Since the folding $g$ is continuous,

$$
p=g(\tilde{p})=\lim _{\lambda \rightarrow 0} g\left(\tilde{q}_{\lambda}\right)
$$

By the Proposition 4.17, each $g\left(\tilde{q}_{\lambda}\right)$ satisfies the chain condition. Observe that the $\Delta$-lengths of the paths $\tilde{p}+\lambda$ are independent of $\lambda$. Since $f$ and $\mathbb{P}$ preserve $\Delta$-lengths 
of piecewise-linear paths and the dilation $h$ changes them by a fixed amount, we can apply the compactness theorem (Theorem 3.30) to conclude that the limiting path $p$ satisfies the chain condition as well.

We now verify that, at certain points, the folded path $p$ satisfies the maximal chain condition.

Proposition 4.19. Under the assumptions of Theorem 4.16 let $\tilde{x}=\tilde{p}(t)$ be such that the folding $f$ restricts to an isometry on the germ $(\tilde{p}, \tilde{x})$. Then the path $p=g(\tilde{p})$ satisfies the maximal chain condition at $x=g(\tilde{x})$.

Proof. Our proof follows Littelmann's arguments in his proof of the PRV Conjecture [L1]. We fold the path $q:=h f(\tilde{p})$ into $\Delta$ inductively.

We subdivide the interval $[0, c]$ as

$$
0=t_{0}<t_{1}<\cdots<t_{k}=c
$$

such that $\left[t_{i}, t_{i+1}\right]$ are maximal subintervals so that $q \|\left[t_{i}, t_{i+1}\right]$ is contained in a Weyl chamber of $W_{\text {sph }}$.

We first apply to $q$ an element $w_{0} \in W_{\text {sph }}$ which sends $q\left(\left[0, t_{1}\right]\right)$ into $\Delta$, so we can assume that this subpath belongs to $\Delta$. Assume that the restriction of $f$ to the germ $\left(\tilde{p}, \tilde{p}\left(t_{1}\right)\right)$ is an isometry. Let $\mu^{\prime}, \eta^{\prime}$ be the vectors $q_{-}^{\prime}\left(t_{1}\right), q_{+}^{\prime}\left(t_{1}\right)$. Then

$$
\mu^{\prime} \sim \eta^{\prime}
$$

see Lemma 4.2. Set $x:=q\left(t_{1}\right)$. The image $\eta$ of the vector $\eta^{\prime}$ under $\mathbb{P}$ is obtained as

$$
d w_{1}\left(\eta^{\prime}\right)
$$

where $w_{1} \in W_{\text {sph }}$ fixes the point $x$ and $\eta$ is the unique vector in the $W_{x}$-orbit of $\eta^{\prime} \in S_{x}$ which points inside $\Delta$. Below we describe $w_{1}$ as a composition of reflections.

Let $R^{\prime}$ denote the root subsystem in $R$ generated by the set of simple roots $\Phi^{\prime}$ which vanish at the point $x$. Let $\Delta^{\prime}$ denote the positive chamber for $W_{x}$ defined via $\Phi^{\prime}$. Then the vector $\eta$ can be described as the unique vector in the $W_{x}$-orbit of $\eta^{\prime}$ (now, regarded as a vector in $\left.V=T_{o}(A)\right)$ which belongs to the interior of $\Delta^{\prime}$. According to Lemma 3.15,

$$
w_{1}=\tau_{m} \circ \cdots \circ \tau_{1} \quad \text { with } \tau_{i}=\tau_{\beta_{i}}, \beta_{i} \in \Phi^{\prime},
$$

for each $i$ so that the sequence of vectors

$$
\left(\eta_{0}=\eta^{\prime}, \eta_{1}:=\tau_{1}\left(\eta_{0}\right), \ldots, \eta_{m}=\tau_{m}\left(\eta_{m-1}\right)=\eta\right),
$$

is a chain in $\left(S, W_{x},-\Delta\right)$ which is maximal as a chain in $\left(S, W_{\mathrm{sph}},-\Delta\right)$. 
We therefore apply the identity transformation to the path $q \mid\left[0, t_{1}\right]$ and the element $w_{1}$ to the path $q \mid\left[t_{1}, c\right]$ to transform the path $q$ into the new path

$$
q_{1}=q\left|\left[0, t_{1}\right] \cup w_{1} \circ q\right|\left[t_{1}, c\right] .
$$

Clearly, $\mathbb{P}(q)=\mathbb{P}\left(q_{1}\right)$ and $\eta$ is the unit vector tangent to $q_{1} \mid\left[t_{1}, c\right]$ at $x$. The above arguments therefore show that $q_{1}$ satisfies the maximal chain condition at the point $x$.

We then proceed to the next point $t_{2}, q_{1}\left(t_{2}\right)$ belongs to the boundary of $\Delta$ and we transform $q_{1}$ to $q_{2}$ by

$$
q_{2}\left|\left[0, t_{2}\right]=q_{1}\right|\left[0, t_{2}\right], \quad q_{2}\left|\left[t_{2}, c\right]=w_{2} \circ q_{1}\right|\left[t_{2}, c\right],
$$

where $w_{2}$ is a certain element of $W_{\text {sph }}$ fixing $q_{1}\left(t_{2}\right)=q_{2}\left(t_{2}\right)$. Therefore $\mathbb{P}\left(q_{2}\right)=$ $\mathbb{P}\left(q_{1}\right)=\mathbb{P}(q)$ and we repeat the above argument.

Definition 4.20. Suppose that $P=\overline{z x} \cup p \cup \overline{y z}$ is a polygon in $A$, where $p:[0,1] \rightarrow A$ is a piecewise-linear path such that $p(0)=x, p(1)=y$. We say that $P$ satisfies the chain condition (simple chain condition and maximal chain condition, respectively) if its subpath $p$ satisfies the chain condition (simple chain condition and maximal chain condition, respectively).

Therefore, as an application of Theorem 4.16 we obtain

Corollary 4.21. Suppose that $T=[\tilde{z}, \tilde{x}, \tilde{y}] \subset X$ is a geodesic triangle, $\tilde{z}$ is a special vertex which belongs to an alcove $a \subset A$. Let $\Delta \subset A$ be a Weyl chamber with the tip $\tilde{z}=o$ and let $P=\operatorname{Fold}_{\Delta}(T)$ be the folding of $T$ into $\Delta$. Then the folded triangle $P$ satisfies the chain condition.

A converse to this corollary will be proven in Theorem 6.9, p. 473; the following is a partial converse to Corollary 4.21 (which is essentially contained in [KLM3], Lemma 7.7):

Corollary 4.22. Let $\Delta \subset A$ be a Weyl chamber with tip o in $X$. Suppose that a polygon $P=\left[o, x_{1}, \ldots, x_{n}\right] \subset \Delta$ satisfies the simple chain condition (at each vertex $\left.x_{i}, 0<i<n\right)$ and

$$
p=\overline{x_{1} x_{2}} \cup \cdots \cup \overline{x_{n-1} x_{n}}
$$

is a billiard path. Then $P$ unfolds to a geodesic triangle $T \subset X$, i.e., $\operatorname{Fold}_{\Delta}(T)=P$.

Proof. Let $f:=$ Fold $_{\Delta}$. We run the argument from the proof of Theorem 4.9 in the reverse; the reader will observe that our argument is essentially the same as in the proof of the Transfer Theorem in [KLM2]. Triangulating the polygon $P$ from the vertex $o$ we obtain geodesic triangles $P_{i}=\left[o, x_{i}, x_{i+1}\right], i=1,2, \ldots, n-1$. Denote 
by $\xi_{i}, \zeta_{i}, \eta_{i} \in \Sigma_{x_{i}}(A)$ the unit tangent vectors to the segments $\overline{x_{i} x_{i-1}}, \overline{x_{i} o}, \overline{x_{i} x_{i+1}}$, respectively.

We unfold $P$ inductively. Set $T_{1}:=P_{1}$; let $A_{1}:=A$, this apartment contains the triangle $T_{1}$. Set $\tilde{x}_{1}:=x_{1}, \tilde{x}_{2}:=x_{2}$,

Suppose that we have constructed apartments $A_{i} \subset X$ and flat triangles $T_{i}=$ $\left[o, \tilde{x}_{i}, \tilde{x}_{i+1}\right] \subset A_{i}, i=1, \ldots, m-1$, so that $T_{i}$ is congruent to $P_{i}(i=1, \ldots, m-1)$ and

$$
\angle\left(\tilde{\xi}_{i}, \tilde{\eta}_{i}\right)=\pi, \quad i=1, \ldots, m-1 .
$$

Here $\tilde{\xi}_{i}, \tilde{\eta}_{i}, \tilde{\zeta}_{i}$ are directions in $\Sigma_{\tilde{x}_{i}}(X)$ which correspond to the directions $\xi_{i}, \eta_{i}, \zeta_{i}$ under the congruences $T_{i} \rightarrow P_{i}$. Our goal is to produce a flat triangle $T_{m} \subset A_{m} \subset X$ so that the above properties still hold.

Since we have a simple chain $\left(-\xi_{m}, \eta_{m}\right)$ in $\left(S_{x_{m}}, W_{x_{m}}\right)$, it follows from Proposition 4.13 that there exists a point $\tilde{\eta}_{m} \in \Sigma_{\tilde{x}_{m}}(X)$ such that

$$
d\left(\tilde{\eta}_{m}, \tilde{\xi}_{m}\right)=\pi, \quad d\left(\tilde{\eta}_{m}, \zeta_{m}\right)=d_{\text {ref }}\left(\eta_{m}, \zeta_{m}\right) .
$$

Let $A_{m}$ denote an apartment in $X$ which contains $\overline{o \tilde{x}_{m}}$ and such that $\tilde{\eta}_{m}$ is tangent to $A_{m}$. Construct a geodesic segment $\overline{\tilde{x}}_{m} \tilde{x}_{m+1} \subset A_{m} \subset X$ whose metric length equals the one of $\overline{x_{m} x_{m+1}}$ and whose initial direction is $\tilde{\eta}_{m}$. This defines a flat triangle

$$
T_{m}=\left[o, \tilde{x}_{m}, \tilde{x}_{m+1}\right] \subset A_{m} .
$$

It is clear from the construction that the triangle $T_{m}$ is congruent to $P_{m}$, in particular, $d_{\text {ref }}\left(o, x_{3}\right)=d_{\text {ref }}\left(o, y_{3}\right)$. Observe also that

$$
\overline{\tilde{x}_{m-1} \tilde{x}_{m}} \cup \overline{\tilde{x}_{m} \tilde{x}_{m+1}}
$$

is a geodesic segment (because $\angle\left(\tilde{\eta}_{m}, \tilde{\xi}_{m}\right)=\pi$ ). See Figure 7 .
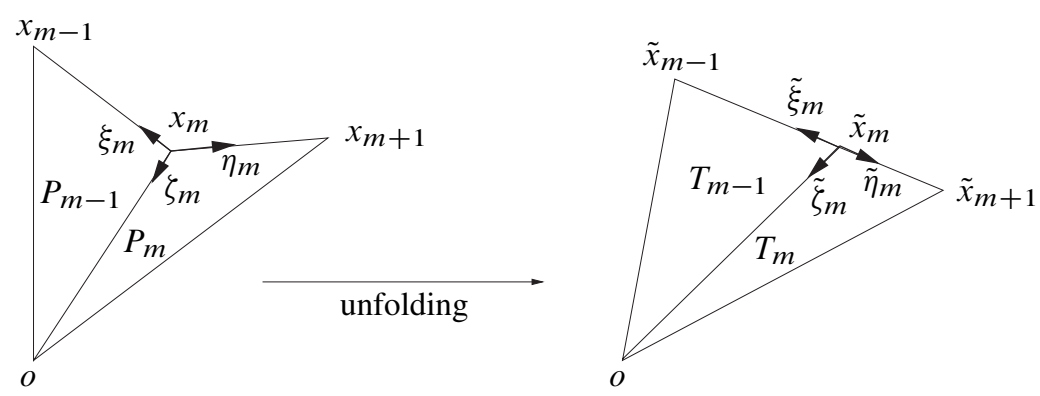

Figure 7. Unfolding a broken triangle.

Therefore, by induction we obtain a geodesic triangle $T=\left[o, x_{1}, \tilde{x}_{n}\right] \subset X$, which is triangulated (from $o$ ) into flat geodesic triangles $T_{i}$ which are congruent to $P_{i}$ 's. We 
claim that $f(T)=P$. For each $i$ the folding $f$ sends the triangle $T_{i}$ to $P_{i}$, according to Proposition 4.7. Therefore $f(T)=P$.

As in the proof of Theorem 4.9, the argument has to be modified in case when $x_{i}=o$ for some $i, x_{i}=p\left(t_{i}\right)$. Then the vector $\mu=p_{-}^{\prime}\left(t_{i}\right)$ belongs to the negative chamber $-\Delta$ and the vector $\lambda=p_{+}^{\prime}\left(t_{i}\right)$ belongs to the positive chamber $\Delta$. Since $p$ is a billiard path, there exists $w \in W_{\text {sph }}$ which sends $\mu$ to $\lambda$. Now the chain and billiard conditions imply that $x_{i}$ is the only break point in $p$. Thus we can take

$$
T:=\overline{o x_{1}} \cup p\left(\left[0, t_{i}\right]\right) \cup w^{-1} p\left(\left[t_{i}, 1\right]\right) \cup w^{-1}\left(\overline{o x_{n}}\right) .
$$

This degenerate geodesic triangle (it is contained in the geodesic through the points $x_{1}, w\left(x_{n}\right)$ ) folds to $P$ under the projection $\mathbb{P}_{\Delta}: A \rightarrow \Delta$.

The same argument as above proves the following generalization of Corollary 4.22.

Corollary 4.23. Suppose that $P$ is a polygon in $\Delta$ which is the composition

$$
\overline{o x} \cup p \cup q \cup \overline{y o} \text {. }
$$

Assume that paths $p, q$ satisfy the simple chain condition. Then there exists a polygon $\widetilde{P} \subset X$ of the form

$$
\overline{o x} \cup \tilde{p} \cup \tilde{q} \cup \overline{\tilde{y} o}
$$

such that $f(\tilde{P})=P, f(\tilde{p})=p, f(\tilde{q})=q, f(\tilde{y})=y$, and $\tilde{p}, \tilde{q}$ are geodesic paths.

We now use our analysis of the folded triangles (polygons) to relate them to the Littelmann triangles (polygons).

\section{Littelmann polygons}

5.1. LS paths. Let $R$ be a root system on a Euclidean vector space $V, W=W_{\text {sph }}$ be the finite Coxeter group associated with $R$, and let $W_{\text {aff }}$ denote the affine Coxeter group associated to $R$. This root system $R$ is actually the coroot system for the one considered by Littelmann in [L2]. Accordingly, we will switch weights to coweights, etc. We pick a Weyl chamber $\Delta$ for $W$, this determines the positive roots and the simple roots in $R$. We get the Euclidean Coxeter complex $\left(A, W_{\text {aff }}\right)$, where $A$ is the affine space corresponding to $V$. Given $x \in A$ let $W_{x}$ denote the stabilizer of $x$ in $W_{\text {aff }}$.

Suppose we are given a vector $\lambda \in \Delta \subset V$, a sequence of real numbers

$$
\underline{a}=\left(a_{0}=0<a_{1}<\cdots<a_{r}=1\right),
$$

and a sequence of vectors in $W \lambda$

$$
\underline{v}=\left(v_{1}, \ldots, v_{r}\right) \text { so that } v_{1}>\cdots>v_{r}
$$

with respect to the order in Definition 3.5. 
Definition 5.1. The pair $(\underline{v}, \underline{a})$ is called a real (billiard) path of the $\Delta$-length $\lambda$.

Definition 5.2 ((P. Littelmann, [L2]). A real path of $\Delta$-length $\lambda$ is called rational if $\lambda$ is a coweight and all numbers $a_{i}$ a rational.

Remark 5.3. Littelmann uses the notion path of type $\lambda$ rather than of the $\Delta$-length $\lambda$.

Set $a_{i}^{\prime}:=a_{i}-a_{i-1}, i=1,2, \ldots, r$. The data $(\underline{v}, \underline{a})$ determines a piecewise-linear path $p \in \mathcal{P}$ whose restriction to each interval $\left[a_{i-1}, a_{i}\right]$ is given by

$$
p(t)=\sum_{k=1}^{i-1} a_{k}^{\prime} v_{k}+\left(t-a_{i-1}\right) v_{i}, \quad t \in\left[a_{i-1}, a_{i}\right] .
$$

Our interpretation of real and rational paths is the one of a broken (oriented) geodesic $L$ in $V$. Each oriented geodesic subsegment of $L$ is parallel to a positive multiple of an element of $W \lambda$, thus $L$ is a billiard path. The break points of the above path are the points

$$
x_{1}=a_{1} v_{1}, \ldots, x_{i}=x_{i-1}+a_{i}^{\prime} v_{i}, \ldots
$$

Since $\sum_{i} a_{i}^{\prime}=1$, is clear that

$$
\text { length }_{\Delta}(L)=\lambda,
$$

in the sense of the definition in Section 2.5. This justifies our usage of the name $\Delta$-length $\lambda$ in the above definitions, rather than Littelmann's notion of type.

Observe that given a piecewise-linear path $p(t) \in \mathcal{P}$ (parameterized with the constant speed) one can recover the nonzero vectors $v_{i} \in V$ and the numbers $a_{i}$ and $a_{i}^{\prime}$.

Definition 5.4 (P. Littelmann, [L2]). A rational path $p(t)$ is called an $L S$ path $^{3}$ if it satisfies a further integrality condition: For each $i=1, \ldots, s-1$ there exists an $a_{i}$-chain for the pair $\left(v_{i}, v_{i+1}\right)$ (in the sense of Definition 3.20).

Observe that since

$$
\eta \geq \tau \Longleftrightarrow-\tau \geq-\eta,
$$

it follows that $p$ is an LS path if and only if $p^{*}$ is.

Theorem 5.5 (P. Littelmann, [L2], Lemma 4.5). Each LS path belongs to $\mathcal{P}_{\mathbb{Z}, \text { loc. }}$

\footnotetext{
${ }^{3}$ A Lakshmibai-Seshadri path.
} 
Our next goal is to give a more geometric interpretation of LS paths. Suppose that $p \in \mathcal{P}$ is a billiard path given by the equation (4), with the vertices

$$
0=x_{0}, x_{1}, \ldots, x_{r} .
$$

At each vertex point $x_{i}, 0<i<r$, we have unit tangent vectors $\xi_{i}, \mu_{i}$ which are tangent to the segments $\overline{x_{i} x_{i-1}}, \overline{x_{i} x_{i+1}}$. Note that at each vertex $x_{i}, 0<i<r$, we have the restricted and unrestricted spherical Coxeter complexes; the positive chamber $\Delta$ in $V$ determines positive chambers $\Delta_{i}$ in the restricted spherical complexes $\left(S_{x_{i}}, W_{x_{i}}\right)$.

Theorem 5.6. A billiard path $p(t)$ of $\Delta$-length $\lambda \in P\left(R^{\vee}\right)$ is an LS path if and only if it is a Hecke path which satisfies the maximal chain condition (cf. Definition 3.26): At each vertex $x_{i}, 0<i<r$ there exists a $\left(S_{x_{i}}, W_{x_{i}},-\Delta_{i}\right)$-chain between $-\xi_{i}$ and $\mu_{i}$, and this chain is maximal as a $\left(S_{x_{i}}, W\right)$-chain.

Proof. Recall that given a nonzero vector $v \in V, \bar{v}$ denotes its normalization $v /|v|$.

It is easy to see (and left to the reader) that if $p(t)$ is a satisfies the above chain condition and $\lambda=$ length $_{\Delta}(p)$ is a coweight, then all numbers $a_{i}$ are rational.

Consider the first break point $x_{1}=x_{0}+a_{1} v_{1}$ of the broken geodesic path $p(t)$. Observe that

$$
\bar{v}_{1}=-\xi_{1}, \quad \bar{v}_{2}=\mu_{1} \in S_{x_{1}} .
$$

According to Proposition 3.24, existence of an $a_{1}$-chain for the pair $\left(v_{1}, v_{2}\right)$ is equivalent to existence of an $\left(S_{x_{1}}, W_{x_{1}}, \Delta_{1}\right)$-chain

$$
\left(\bar{v}_{1}=\eta_{1,0}, \eta_{1,1}, \ldots, \eta_{1, s_{1}}=\bar{v}_{2}\right),
$$

which is maximal in the unrestricted Coxeter complex. Thus the path $p$ satisfies the maximal chain condition at the first break point $x_{1}$ if and only if it satisfies at the point $x_{1}$ the integrality condition from Definition 5.4.

We now proceed to the next break point $x_{2}=x_{1}+a_{2}^{\prime} v_{2}$. We identify normalized vectors $\bar{v}_{1}, \bar{v}_{2}$ with unit vectors in $S_{x_{1}}$. Note that if $p(t)$ is an LS path of the $\Delta$-length $\lambda$, then there exists an element $w_{1} \in W_{x_{1}}$ which sends $\bar{v}_{1}$ to $\bar{\nu}_{2}$. The same is true if $p$ is a Hecke path.

Set $x_{2}^{\prime}:=w_{1}^{-1}\left(x_{2}\right)$. Observe that in both cases of an LS path and a Hecke path,

$$
\overline{x_{0} x_{1}} \cup \overline{x_{1} x_{2}^{\prime}}
$$

is a geodesic segment $\overline{x_{0} x_{2}^{\prime}}$; the corresponding directed segment represents the vector $a_{2} v_{1}$. Let $w_{1}^{\prime} \in W_{\mathrm{sph}}$ denote the linear part of $w_{1}$. Set $x_{2}^{\prime \prime}:=w_{1}^{\prime}\left(x_{2}^{\prime}\right)$. We translate the vectors $\bar{v}_{2}, \bar{v}_{3}$ to the unit tangent sphere $S_{x_{2}^{\prime \prime}}$. The directed segment $\overrightarrow{x_{0} x_{2}^{\prime \prime}}$ represents the vector $a_{2} v_{2}$. See Figure 8 . 


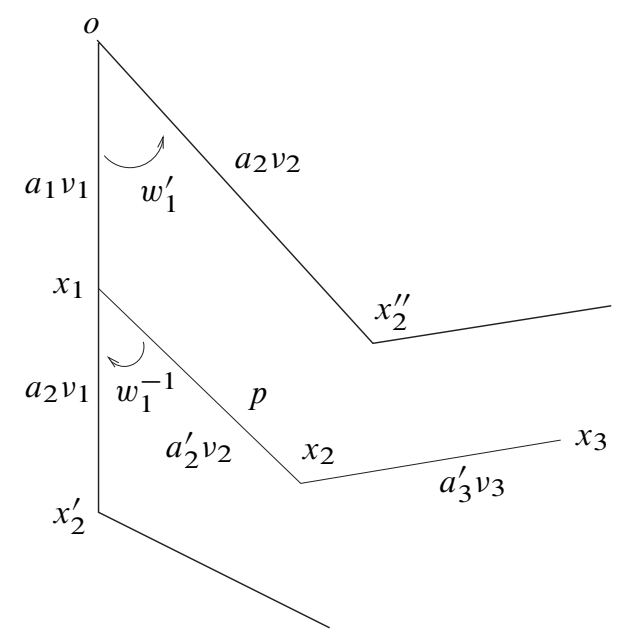

Figure 8. Unbending a path.

We are now again in a position to apply Proposition 3.24 with $a=a_{2}$ : There exists a maximal chain

$$
\left(\bar{v}_{2}=\eta_{2,0}, \eta_{2,1}, \ldots, \eta_{2, s_{2}}=\bar{v}_{3}\right)
$$

if and only if there exists an $a_{2}$-chain for the pair $\left(v_{2}, v_{3}\right)$. The product $w_{1} \circ\left(w_{1}^{\prime}\right)^{-1}$ is a translation in $W_{\text {aff }}$ which carries $x_{2}^{\prime \prime}$ back to $x_{2}$. Therefore it induces an isomorphism of the restricted Coxeter spherical complexes

$$
\left(S_{x_{2}^{\prime \prime}}, W_{x_{2}^{\prime \prime}}\right) \rightarrow\left(S_{x_{2}}, W_{x_{2}}\right)
$$

which carries positive chamber to positive chamber. Hence this translation sends the chain $\left(\eta_{2, i}\right)$ to a maximal chain in $\left(S_{x_{2}}, W_{x_{2}}\right)$.

We continue in this fashion: On the $i$-th step we "unbend" the broken geodesic

$$
\overline{x_{0} x_{1}} \cup \cdots \cup \overline{x_{i-1} x_{i}}
$$

to a directed geodesic segment $\overrightarrow{x_{0} x_{i}^{\prime}}$ representing the vector $a_{i} v_{1}$, then apply an

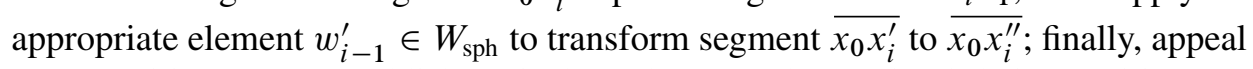
to Proposition 3.24 to establish equivalence between the maximal chain condition and the LS path axioms.

As a corollary of Theorem 5.6 we obtain

Corollary 5.7. Let $T=[z, x, y] \subset X$ be a geodesic triangle and $f=$ Fold $_{z, h, \Delta}$ be a folding into the Weyl chamber. Set $\beta:=d_{\Delta}(x, y)$. Assume that $T^{\prime}=f(T)$ is 
such that $f(x), f(y)$ and all break points of the broken geodesic $f(\overline{x y})$ are special vertices. Then $f(\overline{x y})$ is an LS path of the $\Delta$-length $k \beta$. Here $k$ is the conformal factor of the dilation $h$.

5.2. Root operators. With each simple root $\alpha \in \Phi$, Littelmann [L2] associates raising and lowering root operators $e_{\alpha}$ and $f_{\alpha}$ acting $\mathcal{P}$ as follows.

Recall that given a path $p(t)$ and a root $\alpha$ we have the height function $h_{\alpha}(t):=$ $\alpha(p(t))$. The number $m_{\alpha}$ is the minimal value of $h_{\alpha}$ on $[0,1]$.

If $m_{\alpha}>-1$ then $e_{\alpha}$ is not defined on $p$. Otherwise let $t_{1}$ be the minimal $t$ for which $h_{\alpha}(t)=m_{\alpha}$ and let $t_{0} \in\left[0, t_{1}\right]$ be maximal such that $h_{\alpha}(t) \geq m_{\alpha}+1$ for all $t \in\left[0, t_{0}\right]$.

The operator $e_{\alpha}$ will not change the path $p$ for $t \in\left[0, t_{0}\right]$ and, as far as $\left[t_{1}, 1\right]$ is concerned, the path $p \mid\left[t_{1}, 1\right]$ will change only by a translation in $W_{\text {aff }}$ along the line $L_{\alpha}$ parallel to the vector $\alpha^{\vee}$. Thus it remains to describe the path $q=e_{\alpha}(p)$ on $\left[t_{0}, t_{1}\right]$. If $h_{\alpha}$ were not to have any local minima on $\left[t_{0}, t_{1}\right]$ then $\left.q\right|_{\left[t_{0}, t_{1}\right]}$ would be obtained by the reflection

$$
\left.q\right|_{\left[t_{0}, t_{1}\right]}:=\left.\tau_{\alpha} \circ p\right|_{\left[t_{0}, t_{1}\right]}
$$

and we would set

$$
q:=\left.\left.\left.p\right|_{\left[0, t_{0}\right]} * \tau_{\alpha} \circ p\right|_{\left[t_{0}, t_{1}\right]} * p\right|_{\left[t_{1}, 1\right]} .
$$

(Here we treat the paths resulting from the restriction of $p$ to subintervals of $[0,1]$ as elements of $\mathcal{P}$, according to the convention in Section 2.)

This is the definition of $e_{\alpha}$ of [L1], however the definition of $e_{\alpha}$ which we will need in this paper is the more refined one of [L2]. Call a subinterval $[s, u] \subset\left[t_{0}, t_{1}\right]$ a spike if it is a maximal interval satisfying

$$
h_{\alpha}(s)=h_{\alpha}(u)=\min \left(h_{\alpha} \mid[s, u]\right) .
$$

Thus $h_{\alpha} \mid\left[t_{0}, t_{1}\right]$ is decreasing on the complement to the union of spikes. The restriction of $q$ to each spike is obtained from $p$ by a translation along $L_{\alpha}$. The restriction to each subinterval disjoint from a spike is obtained by a reflection. To be more precise, subdivide the interval $\left[t_{0}, t_{1}\right]$ into

$$
\left[t_{0}, s_{1}\right] \cup\left[s_{1}, s_{2}\right] \cup \cdots \cup\left[s_{k}, t_{1}\right],
$$

where the spike and non-spike intervals alternate. Observe that $\left[t_{0}, s_{1}\right],\left[s_{k}, t_{1}\right]$ are not spikes. Then

$$
q:=\left.\left.\left.p\right|_{\left[0, t_{0}\right]} * \tau_{\alpha}\left(\left.p\right|_{\left[t_{0}, s_{1}\right]}\right) * p\right|_{\left[s_{1}, s_{2}\right]} * \cdots * \tau_{\alpha}\left(\left.p\right|_{\left[s_{k}, t_{1}\right]}\right) * p\right|_{\left[t_{1}, 1\right]} .
$$

Note that the operator $e_{\alpha}$ changes the geometry of the path $p$ by an isometry near every point $p(t)$ which is neither a point of local minimum for $h_{\alpha}$ nor is a point where 
$h_{\alpha}(t)=m_{\alpha}-1$. Otherwise the local change is done by a "bending" with respect to a hyperplane parallel to $H_{\alpha}$. These hyperplanes are not necessarily walls of $W_{\text {aff }}$. However, if all local minimal values of $h_{\alpha}$ belong to $\mathbb{Z}$, these hyperplanes are indeed walls and we obtain:

For each path $p \in \mathcal{P}_{\mathbb{Z}, \text { loc }}$ and for each simple root $\alpha$, the path $q=e_{\alpha}(p)$ satisfies the following: The interval $[0,1]$ can be subdivided into subintervals $\left[s_{i}, s_{i+1}\right]$ such that the restriction $q \mid\left[s_{i}, s_{i+1}\right]$ is obtained from the restriction of $p$ by post-composition with an element of $W_{\text {aff }}$.

The lowering operators $f_{\alpha}$ are defined analogously to the raising operators; we refer the reader to [L2] for the precise definition. (See however Property 1 below.) At this stage we note only that $f_{\alpha}$ is undefined on $p$ iff $m_{\alpha}>h_{\alpha}(1)-1$. Let $\mathscr{E}$ be the semigroup generated by $e_{\alpha}$ 's, let $\mathcal{F}$ be the semigroup generated by $f_{\alpha}$ 's and let $\mathcal{A}$ be the semigroup generated by all root operators. The semigroups contain the identity operator by default. For each $\phi \in \mathcal{A}$ let $\operatorname{Dom}(\phi)$ denote the domain of $\phi$.

Remark 5.8. In fact, Littelmann extends the operators $f_{\alpha}, e_{\alpha}$ to the entire $\mathcal{P}$ by declaring $f_{\alpha}(p)=\mathbf{0}$ for all $p$ for which $f_{\alpha}$ is undefined. However we will not need this extension in the present paper.

Below we list certain properties of the root operators. Most of them are either clear from the definition or are proven in [L2]. Most proofs that we present are slight modifications of the arguments in [L2].

Property 1 (P. Littelmann, [L2], Lemma 2.1 (b, e)).

$$
\begin{aligned}
e_{\alpha} \circ f_{\alpha}(p) & =p \text { if } p \in \operatorname{Dom}\left(f_{\alpha}\right), \\
f_{\alpha} \circ e_{\alpha}(p) & =p \text { if } p \in \operatorname{Dom}\left(e_{\alpha}\right), \\
e_{\alpha}\left(p^{*}\right) & =\left(f_{\alpha}(p)\right)^{*}, \\
\left(e_{\alpha}(p)\right)^{*} & =f_{\alpha}\left(p^{*}\right),
\end{aligned}
$$

the latter two equations could be taken as the definition of $f_{\alpha}$.

Property 2 ([L2], Lemma 2.1). For each $p \in \operatorname{Dom}\left(e_{\alpha}\right) \cap \mathcal{P}$,

$$
\begin{gathered}
m_{\alpha}\left(e_{\alpha}(p)\right)=m_{\alpha}(p)+1, \\
p \in \operatorname{Dom}\left(e_{\alpha}^{N}\right) \Longleftrightarrow N<\left|m_{\alpha}\right| .
\end{gathered}
$$

Property 3. Suppose that $p$ is a path in $\mathcal{P}_{\mathbb{Z}}$ which does not belong to the domain of any $e_{\alpha}, \alpha \in \Phi$. Then $p$ is contained in $\Delta$. Indeed, for each simple root $\alpha$ we have to have $m_{\alpha}(p)>-1$. Since $p \in \mathcal{P}_{\mathbb{Z}}, m_{\alpha}(p)=0$. Thus $p \in \mathcal{P}^{+}$.

Property 4 ([L2], Proposition 3.1 (a, b)). For each $\alpha \in \Phi$, $\operatorname{Dom}\left(f_{\alpha}\right) \cap \mathcal{P}_{\mathbb{Z}}$ is open and $f_{\alpha} \mid \mathcal{P}_{\mathbb{Z}}$ is continuous. 
Property 5 ([L2], §7, Corollary 1 (a)). Let $p \in \mathcal{P}^{+}$and $\phi$ be a composition of lowering operators defined on $p$. Then $\phi(p) \in \mathcal{P}_{\mathbb{Z}}$.

Property 6. Combining Properties 4 and 5 we conclude that for each $f \in \mathcal{F}$, $\operatorname{Dom}(\boldsymbol{f}) \cap \mathcal{P}^{+}$is open and $\boldsymbol{f} \mid \mathcal{P}^{+}$is continuous.

Property 7 ([L2], Corollary 3, p. 512). $p$ is an LS path of the $\Delta$-length $\lambda$ if and only if there exists $f \in \mathscr{F}$ such that

$$
p=f\left(\pi_{\lambda}\right)
$$

Property 8 ([L2], Corollary 2 (a), p. 512). The set of LS paths of the given $\Delta$-length is stable under $\mathcal{A}$.

Property 9. Suppose that $p \in \mathcal{P}, t \in[0,1], \alpha \in \Phi$ and $x:=p(t)$ satisfy

$$
\alpha(x) \in \mathbb{Z}, \quad p_{-}^{\prime}(t) \gtrsim_{x} p_{+}^{\prime}(t) .
$$

Then the path $q=e_{\alpha}(p)$ also satisfies

$$
q_{-}^{\prime}(t) \varpi_{y} q_{+}^{\prime}(t)
$$

for $y=q(t)$.

Proof. If $h_{\alpha}(t) \neq m_{\alpha}, m_{\alpha}-1$, the germs of the paths $p$ and $q$ at $t$ differ by a translation. Thus the conclusion trivially holds in this case. The same argument applies if $h_{\alpha}(t)=m_{\alpha}-1$ and

$$
\alpha\left(p_{-}^{\prime}(t)\right) \geq 0, \quad \alpha\left(p_{+}^{\prime}(t)\right) \leq 0 .
$$

The nontrivial cases are:

(1) $h_{\alpha}(t)=m_{\alpha}-1, \alpha\left(p_{-}^{\prime}(t)\right) \leq 0, \alpha\left(p_{+}^{\prime}(t)\right) \leq 0$. In this case the assertion follows from Lemma 3.6 with $v=p_{-}^{\prime}(t), \mu=p_{+}^{\prime}(t)$.

(2) $h_{\alpha}(t)=m_{\alpha}, \alpha\left(p_{-}^{\prime}(t)\right) \leq 0, \alpha\left(p_{+}^{\prime}(t)\right) \geq 0$. In this case the assertion follows from Lemma 3.19 with $v=p_{-}^{\prime}(t), \mu=p_{+}^{\prime}(t)$.

Property 10. Suppose that $p=p_{1} * p_{2}$ where $p_{1} \in \mathcal{P}^{+}$. Then for each $e \in \mathcal{E}$ defined on $p$ we have

$$
e(p)=p_{1} * e\left(p_{2}\right)
$$

Proof. It suffices to prove this for $e=e_{\alpha}, \alpha \in \Phi$. In the latter case it follows directly from the definition of the operator $e_{\alpha}$. 
The next property is again clear from the definition.

Property 11. Suppose that

$$
\boldsymbol{e}=e_{\beta_{m}} \circ \cdots \circ e_{\beta_{1}}
$$

where $\beta_{i} \in \Phi, p \in \operatorname{Dom}(\boldsymbol{e})$. Set

$$
p_{i}:=e_{\beta_{i}} \circ \cdots \circ e_{\beta_{1}}(p), \quad i=1, \ldots, m .
$$

Then for each $T \in[0,1]$, the sequence of vectors

$$
\left(p_{+}^{\prime}(T),\left(p_{1}\right)_{+}^{\prime}(T), \ldots,\left(p_{m}\right)_{+}^{\prime}(T)\right),
$$

after deleting equal members, forms a chain.

Lemma 5.9. Given a path $p$ there are only finitely many operators $\boldsymbol{e} \in \mathcal{E}$ which are defined on $p$.

Proof. Break the path $p$ as the concatenation

$$
p_{1} * \cdots * p_{s}
$$

of geodesic paths, each of which is contained in a single alcove, and let $T_{i} \in[0,1]$ be such that $p\left(T_{i}\right)=p_{i}(1 / 2)$; set $T_{0}:=0$. Then for each $e_{\alpha} \in \mathcal{E}$ defined on $p$ there exists $i$ such that the derivatives of $e_{\alpha}(p)$ and $p$ at $T_{i}$ are not the same. Moreover,

$$
q=e_{\alpha}(p)=q_{1} * \cdots * q_{s},
$$

where each $q_{i}$ is a geodesic path contained in an alcove. Consider the vector

$$
L(p):=\left(\ell\left(p^{\prime}(0)\right), \ell\left(p^{\prime}\left(T_{1}\right)\right), \ldots, \ell\left(p^{\prime}\left(T_{s}\right)\right)\right) \in(\mathbb{N} \cup\{0\})^{s+1},
$$

where $\mathbb{N}^{s+1}$ is given the lexicographic order and $\ell$ is the length function on $W_{\mathrm{sph}^{-}}$ orbits induced from the word metric on $W_{\text {sph }}$ as in Proposition 3.7. Then, by combining Proposition 3.7 and Property 11 above, for each $\alpha \in \Phi$,

$$
L\left(e_{\alpha}(p)\right)<L(p) .
$$

The lemma follows.

5.3. Generalized LS paths. In this paper we will need two generalizations of the concept of an LS path; the first one will be used in the proof of the saturation theorem (Section 7), the second one will be applied in Section 6.1 for the proof of the unfolding theorem. Although we will use the name generalized LS path for both generalizations, it will be clear from the context which generalization is being referred to. 
The first generalization $\mathscr{L} \boldsymbol{S}_{1}{ }^{4}$ Suppose we are given a collection of LS paths $p_{i}$ of the $\Delta$-length $\lambda_{i} \in \Delta \cap P\left(R^{\vee}\right), i=0, \ldots, m$. We will use the notation

$$
\underline{\lambda}=\left(\lambda_{0}, \ldots, \lambda_{m}\right)
$$

and

$$
\lambda:=\sum_{i=0}^{m} \lambda_{i} .
$$

Remark 5.10. Actually, for our main application it will suffice to consider $\lambda_{i}$ 's which are multiples of the fundamental coweights $\varpi_{i}$. Therefore such paths are automatically generalized Hecke paths as defined in Definition 3.29.

Definition 5.11. The concatenation

$$
p=p_{0} * p_{1} * \cdots * p_{m}
$$

is called a generalized LS path with length ${ }_{\Delta}(p)=\underline{\lambda}$ if

$$
p_{i}^{\prime}(1) \gtrsim p_{i+1}^{\prime}(0)
$$

for each $i=0, \ldots, m-1$. The set of such generalized LS paths is denoted $\mathscr{L} \mathscr{S}_{1}$.

This definition is a very special case of the one used by Littelmann in [L3] under the name of a locally integral concatenation.

Recall that according to the definition of $\Delta$-length,

$$
\lambda=\text { length }_{\Delta}(p) .
$$

Observe that each LS path $p$ satisfies the above definition, since

$$
p_{i}^{\prime}(1) \geq p_{i+1}^{\prime}(0)
$$

for each $i$.

Example 5.12. Suppose that $u, v$ are dominant coweights. Then $p=\pi_{u} * \pi_{v}$ is a generalized LS path.

The second generalization $\mathscr{L}_{2} .{ }^{5}$ Suppose that $p_{1}, p_{2} \in \mathcal{P}$ appear as

$$
\begin{array}{ll}
p_{1}=\left.\tilde{p}_{1}\right|_{[0, a]}, & 0<a<1, \\
p_{2}=\left.\tilde{p}_{2}\right|_{[b, 1]}, & 0<b<1,
\end{array}
$$

\footnotetext{
${ }^{4}$ This generalization of LS paths will be used in the proof of the saturation theorem.

${ }^{5}$ This notion of generalized LS path will be used only to unfold Hecke paths.
} 
where $\tilde{p}_{1}, \tilde{p}_{2}$ are LS paths, $a, b \in \mathbb{Q}$. (See Section 2.2 for the definition of $\left.\tilde{p}_{1}\right|_{[0, a]}$ and $\left.\tilde{p}_{2}\right|_{[b, 1]}$.) Define the path $p:=p_{1} * p_{2}$. Assume that

$$
p_{1}^{\prime}(1) \triangleright p_{2}^{\prime}(0)
$$

in other words, if $t$ is such that $p(t)=p_{1}(1)$ then

$$
p_{-}^{\prime}(t) \triangleright p_{+}(t)
$$

Definition 5.13. The concatenation $p$ is called a generalized LS path if the concatenation point $p_{1}(1)$ is a regular point ${ }^{6}$ of $\left(A, W_{\text {aff }}\right)$ and $p(1) \in P\left(R^{\vee}\right)$.

The set of such generalized LS paths is denoted $\mathscr{L} \delta_{2}$.

Example 5.14. Suppose that $u \in \Delta, v \in V$ are such that $u, v \in P\left(R^{\vee}\right) \otimes \mathbb{Q}$, $u+v \in P\left(R^{\vee}\right) \cap \Delta$ and the head of the vector $u$ is a regular point in $\left(A, W_{\text {aff }}\right)$. Then $p=\pi_{u} * \pi_{v} \in \mathscr{L} \oiint_{2}$.

This definition is again a very special case of the one given by Littelmann in [L2], 5.3. Littelmann does not assume that $p_{1}(1)$ is regular, but instead imposes certain chain conditions at this point.

\section{Properties of generalized LS paths}

Property 0. If $p \in \mathscr{L} \mho_{1}$ then $p^{*}$ is also in $\mathscr{L} \mho_{1}$.

Proof. Represent $p$ as a concatenation $p_{1} * \cdots * p_{m}$ of LS paths as in the Definition 5.11. Then

$$
p^{*}=\left(p_{m}^{*}\right) * \cdots *\left(p_{1}^{*}\right),
$$

where each path $p_{i}^{*}$ is again an LS path. The assertion follows from Lemma 3.18.

Property 1. $\mathscr{L} \oiint_{2}$ is stable under the root operators [L2], Lemma 5.6, second assertion. In particular, suppose that $p$ is as in Example 5.14. Then for each $\boldsymbol{f} \in \mathcal{F}$ (defined on $p), \boldsymbol{f}(p)$ is a generalized LS path of the $\Delta$-length $\lambda=u+v$.

Property 2. $\mathscr{L} \delta_{1}$ is stable under the root operators.

Proof. Suppose that $p=p_{1} * \cdots * p_{m}$ is a concatenation of LS paths as above and $e_{\alpha}$ is a raising operator. In particular, for each $i$ we have a vector $u_{i}$ so that

$$
p_{i}^{\prime}(1) \geq u_{i} \sim p_{i+1}^{\prime}(0)
$$

\footnotetext{
${ }^{6}$ I.e., it does not belong to any wall.
} 
For each $i, e_{\alpha}\left(p_{i}\right)$ is again a Littelmann path. Therefore $e_{\alpha}(p)$ is a concatenation of LS paths $q_{1} * \cdots * q_{m}$. We have to verify that for each $i$ there is a vector $v_{i} \in V$ so that

$$
q_{i}^{\prime}(1) \geq v_{i} \sim q_{i+1}^{\prime}(0) .
$$

This however follows from the Property 9 in the previous section. To check that $\mathscr{L} \oiint_{1}$ is preserved by $f_{\alpha}$ we use that $q \in \mathscr{L} \mathscr{S}_{1} \Longleftrightarrow q^{*} \in \mathscr{L} \mathscr{S}_{1}$ and

$$
f_{\alpha}(p)=\left(e_{\alpha}\left(p^{*}\right)\right)^{*} .
$$

Property 3. $\mathscr{L} \mathscr{S}_{1}$ and $\mathscr{L} \mathscr{S}_{2}$ are contained in $\mathcal{P}_{\mathbb{Z}, \text { loc }}$. For $\mathscr{L}_{1}$ it is immediate since, by Theorem 5.5, the set of LS paths is contained in $\mathcal{P}_{\mathbb{Z} \text {,loc }}$. For $\mathscr{L}_{2}$ it is a special case of [L2], Lemma 5.5.

Property 4. Suppose that $p \in \mathscr{L} \mho_{1}$. Then there exists an element $\boldsymbol{e} \in \mathcal{E}$ defined on $p$ such that $q=e(p) \in \mathcal{P}^{+}$.

Proof. If $m_{\alpha_{1}}(p) \leq-1$ then we apply a power $e_{\alpha_{1}}^{k_{1}}$ to $p$ so that $q_{1}:=e_{\alpha_{1}}^{k_{1}}(p)$ satisfies $m_{\alpha_{1}}\left(q_{1}\right)>-1$. However, since $e_{\alpha_{1}}^{k_{1}}(p) \in \mathscr{L} \delta_{1} \subset \mathcal{P}_{\mathbb{Z} \text {,loc }}$, it follows that $q_{1} \in \mathcal{P}_{\mathbb{Z} \text {,loc }}$ and so $m_{\alpha_{1}}\left(q_{1}\right)=0$. We then apply a power of $e_{\alpha_{2}}$ to $q_{1}$, etc. According to Lemma 5.9 , this process must terminate. Therefore, in the end we obtain a path

$$
\boldsymbol{e}(p)=q
$$

which does not belong to the domain of any raising operator. Since $q \in \mathcal{P}_{\mathbb{Z} \text {,loc }}$ it follows that $q$ is entirely contained in $\Delta$.

Recall [Bo], Chapter VI, Section 10, that if a root system $R$ spans $V$ then each dominant coweight $\lambda \in \Delta$ is a positive integral combination

$$
\lambda=\sum_{i=1}^{l} n_{i} \varpi_{i},
$$

where $\varpi_{i}$ are fundamental coweights. This assertion (as it stands) is false without the above assumption on $V$. In the general case we have

$$
\lambda=\lambda^{\prime}+\sum_{i=1}^{l} n_{i} \varpi_{i},
$$

where $\lambda^{\prime} \in V^{\prime}, n_{i} \in \mathbb{N} \cup\{0\}$. As an alternative the reader can restrict the discussion to semisimple groups only when $V^{\prime}=0$. 
Convention 5.15. From now on we will be assuming that in Definition 5.11

$$
\lambda_{j}=k_{j} \varpi_{j}, \quad k_{j} \in \mathbb{N},
$$

for each $j=1, \ldots, m$, where $\varpi_{j}$ is the $j$-th fundamental coweight, and

$$
\lambda_{0} \in V^{\prime} \text {. }
$$

Then the subpath $p_{0}$ is necessarily geodesic.

Lemma 5.16. Suppose that $p \in \mathscr{L}_{1} \cap \mathcal{P}^{+}$is a generalized LS path with $\underline{\text { length }}_{\Delta}(p)=\underline{\lambda}$. Then

$$
p=\pi_{\lambda_{0}} * \cdots * \pi_{\lambda_{m}}
$$

Proof. Represent $p$ as the concatenation $p=p_{0} * p_{1} * \cdots * p_{m}$ of maximal LS subpaths. The geodesic subpath $p_{0}$ clearly equals $\pi_{\lambda_{0}}$. Since $p_{1}$ is an LS path and $p_{1}^{\prime}(0) \in \Delta$, we see that $p_{1}$ is a geodesic path (see Corollary 3.9) which therefore equals $\pi_{\lambda_{1}}$. Moreover, because

$$
p_{1}^{\prime}(1) \geq u_{1} \sim p_{2}^{\prime}(0),
$$

it follows that $u_{1}=p_{1}^{\prime}(1)$ and thus $p_{1}^{\prime}(1)=\lambda_{1} \sim p_{2}^{\prime}(0)$. Let $x_{1}:=p_{1}(1)$; this point lies on the boundary face of $\Delta$ which does not contain $\lambda_{2}$. Note that the vector $p_{2}^{\prime}(0)$, regarded as an element of $T_{x_{1}}(A)$, points inside the Weyl chamber $\Delta$ (for otherwise $p$ is not contained in $\Delta$ ). On the other hand, since $\lambda_{1} \sim p_{2}^{\prime}(0)$, the vector $p_{2}^{\prime}(0)$ belongs either to $\Delta$ or to the Weyl chamber

$$
\tau_{\beta_{2}}(\Delta)
$$

adjacent to $\Delta$. Since $p \in \mathcal{P}^{+}$, it is clear that $p_{2}^{\prime}(0) \in \Delta$. Thus $p_{2}$ is the geodesic path $\pi_{\lambda_{2}}$. Continuing in this fashion we conclude that

$$
p=\pi_{\lambda_{0}} * \pi_{\lambda_{1}} * \cdots * \pi_{\lambda_{m}} .
$$

Theorem 5.17. Suppose that $p$ is a generalized LS path in the sense of $\mathscr{L} 8_{1}$ with $\underline{\text { length }}_{\Delta}(p)=\underline{\lambda}$ (satisfying convention 5.15). Then there exists $\boldsymbol{f} \in \mathcal{F}$ such that

$$
p=f\left(\pi_{\lambda_{0}} * \cdots * \pi \lambda_{m}\right)
$$

Proof. If $p \in \mathcal{P}^{+}$then we are done. Otherwise, by combining Lemma 5.16 with the Property 4, we find an $\boldsymbol{e} \in \mathcal{E}$,

$$
\boldsymbol{e}=e_{\alpha_{1}}^{k_{1}} \circ \cdots \circ e_{\alpha_{n}}^{k_{n}},
$$

such that $p \in \operatorname{Dom}(\boldsymbol{e})$ and $\boldsymbol{e}(p)=q \in \mathcal{P}^{+}$. Therefore

$$
q=\pi_{\lambda_{0}} * \pi_{\lambda_{1}} * \cdots * \pi_{\lambda_{m}}
$$

and thus the composition

$$
\boldsymbol{f}=f_{\alpha_{n}}^{k_{n}} \circ \cdots \circ f_{\alpha_{1}}^{k_{1}}
$$

satisfies $p=\boldsymbol{f e}(p)=\boldsymbol{f}\left(\pi_{\lambda_{0}} * \pi_{\lambda_{1}} * \cdots * \pi_{\lambda_{m}}\right)$. 
5.4. Path model for the representation theory of Lie groups. Suppose that

$$
p(t) \in \mathscr{L} \delta_{1} \cup \mathscr{L} \delta_{2}
$$

is a generalized LS path with length ${ }_{\Delta}(p)=\beta$ and

$$
\text { length }_{\Delta}(p)=\underline{\lambda} \text {. }
$$

Suppose that $\alpha \in P\left(R^{\vee}\right)$ is such that $\alpha+p(t)$ is contained in $\Delta$. Then $\alpha$ and $p$ define a polygon

$$
P:=\overline{o y_{0}} \cup(p+\alpha) \cup \overline{y_{n} o} \subset \Delta
$$

where $\alpha=\overrightarrow{o y_{0}}, y_{n}=\alpha+p(1)$. Let $\gamma$ denote the vector $\overrightarrow{o y_{n}}$; then $\gamma$ is also a dominant coweight. Recall that the contragredient dominant coweight $\gamma^{*} \in \Delta$ is obtained by projecting the vector $-\gamma$ to the Weyl chamber $\Delta$ by the projection $\mathbb{P}: V \rightarrow \Delta$.

Definition 5.18. (1) A polygon $P$ above is called a (broken) Littelmann polygon with the $\Delta$-side lengths $\alpha, \beta, \gamma^{*}$.

(2) If $p(t)$ is an LS path then $P$ is called a (broken) Littelmann triangle with the $\Delta$-side lengths $\alpha, \beta, \gamma^{*}$.

Pick a lattice $L$ such that

$$
Q\left(R^{\vee}\right) \subset L \subset P\left(R^{\vee}\right) .
$$

Then there exists a unique connected semisimple complex Lie group $G^{\vee}$ with the root system $R^{\vee}$ and the character lattice $L$ of the maximal torus $T^{\vee} \subset G^{\vee}$. Recall that irreducible representations $V$ of $G^{\vee}$ are parameterized by their dominant weights, $V=V_{\lambda}, \lambda \in \Delta \cap L$.

Pick a path $q \in \mathcal{P}^{+}$such that $q(1)=\beta$. Then, according to [L2], decomposition formula, p. 500, we have

Theorem 5.19. The tensor product $V_{\alpha} \otimes V_{\beta}$ contains $V_{\gamma}$ as a subrepresentation if and only if there exists a path $p \in \mathcal{F}(q)$ such that $\pi_{\alpha} * p \in \mathcal{P}^{+}$and $\pi_{\alpha} * p(1)=\gamma$.

Remark 5.20. Littelmann works with simply-connected group $G^{\vee}$ and weights $\alpha, \beta, \gamma$ in $P\left(R^{\vee}\right)$. The statement for non-simply-connected groups trivially follows from the simply-connected case.

In particular, since $p$ is an LS paths of the $\Delta$-length $\beta$ if and only if $p \in \mathcal{F}\left(\pi_{\beta}\right)$, we have the following result.

Theorem 5.21 ([L1], [L2]). The tensor product $V_{\alpha} \otimes V_{\beta}$ contains $V_{\gamma}$ as a subrepresentation if and only if there exists a (broken) Littelmann triangle in $\Delta \subset V$, with the $\Delta$-side lengths $\alpha, \beta, \gamma^{*}$.

In other words, $V_{\gamma} \subset V_{\alpha} \otimes V_{\beta}$ if and only if there exists an LS path $p$ of $\Delta$-length $\beta$ such that 
(1) $\pi_{\alpha} * p \in \mathcal{P}^{+}$;

(2) $p(1)+\alpha=\gamma$.

We will apply Theorem 5.19 as follows. Represent the vector $\beta$ as the integer linear combination of fundamental coweights

$$
\beta=\sum_{i=1}^{n} k_{i} \varpi_{i} .
$$

We reorder the fundamental coweights so that $k_{i}>0$ for all $i=1, \ldots, m$ and $k_{i}=0$, $i \geq m+1$. Set $\lambda_{i}:=k_{i} \varpi_{i}, 1 \leq i \leq m$ and let $\underline{\lambda}=\left(\lambda_{1}, \ldots, \lambda_{m}\right)$. Therefore the path

$$
\pi_{\underline{\lambda}}:=\pi_{\lambda_{1}} * \cdots * \pi_{\lambda_{m}}
$$

belongs to $\mathscr{L} \oiint_{1}$ and $\pi_{\underline{\lambda}}(1)=\beta$.

Corollary 5.22. The tensor product $V_{\alpha} \otimes V_{\beta}$ contains $V_{\gamma}$ as a subrepresentation if and only if there exists a generalized LS path $p$ so that

(1) length $_{\Delta}(p)=\underline{\lambda}$;

(2) $\pi_{\alpha} * p \in \mathcal{P}^{+}$;

(3) $\pi_{\alpha} * p(1)=\gamma$.

Proof. Set

$$
q=\pi_{\underline{\lambda}} .
$$

According to Theorems 5.17 and Property 2 of generalized LS paths (Section 5.3), $p \in \mathcal{P}$ is a generalized LS path with length ${ }_{\Delta}(p)=\underline{\lambda}$ if and only if $p \in \mathcal{F}(q)$. Now the assertion follows from Theorem 5.19.

Combining Corollary 4.21, Theorem 5.6 and Theorem 5.21 we obtain

Corollary 5.23. Suppose that $X$ is a thick Euclidean building modeled on the Coxeter complex $\left(A, W_{\text {aff }}\right)$. Let $\alpha, \beta, \gamma^{*} \in L$ be dominant coweights. Suppose that a $\subset A$ is an alcove containing a special vertex $o, T=[o, x, y] \subset X$ is a geodesic triangle with the special vertices and the $\Delta$-side lengths $\alpha, \beta, \gamma^{*}$. Assume also that the broken side Fold $_{a, A}(\overline{x y})$ of the folded triangle

$$
\operatorname{Fold}_{a, A}(T)
$$

has breaks only at the special vertices of $A$. Then

(1) the folded triangle $T^{\prime}=\operatorname{Fold}_{o, \mathrm{id}, \Delta}(T) \subset \Delta$ is a Littelmann triangle;

(2) $V_{\gamma} \subset V_{\alpha} \otimes V_{\beta}$. 
Proof. Indeed, according to Corollary 4.21, the folded triangle $T^{\prime}$ satisfies the chain condition. Each break point $x_{i}$ on the broken side of $T^{\prime}$ is

(1) either a special vertex, in which case it satisfies maximal chain condition by Remark 3.25, or

(2) Fold ${ }_{a, A}(\overline{x y})$ is geodesic at the point corresponding to $x_{i}$, so the chain at $x_{i}$ can be chosen to be maximal by Proposition 4.19.

Hence Theorem 5.6 implies that $T^{\prime}$ is a Littelmann triangle. The second assertion now follows from Theorem 5.21.

Of course, the assumption that the break points occur only at the special vertices is very restrictive. In Section 7 we will get rid of this assumption at the expense of dilation of the side lengths.

\section{Unfolding}

The goal of this section is to establish an intrinsic characterization of folded triangles as the broken billiard triangles satisfying the chain condition. We first prove this characterization for Littelmann triangles and then, using this, give a general proof.

Throughout this section we assume that $X$ is a thick locally compact Euclidean building modeled on the Coxeter complex $(A, W), \Delta \subset A$ is a Weyl chamber with tip $o$. Let $g: X \rightarrow \Delta$ denote the folding Fold $\Delta$.

Let $T \subset \Delta$ be a billiard triangle which is the composition

$$
T=\overrightarrow{o x} \cup r \cup \overline{y o},
$$

where $r(t)=p(t)+\alpha, \alpha=\overrightarrow{o x}$ and $p \in \mathcal{P}$ is a Hecke path. Thus $T$ has the geodesic sides $\overline{o x}, \overline{o y}$ and the broken side $r$. We set $\gamma:=\overrightarrow{o y}$ and let $\beta \in \Delta$ denote $\Delta$-length of the path $p$.

\subsection{Unfolding Littelmann triangles}

Theorem 6.1. Suppose that, in addition, $T$ is a Littelmann triangle, i.e., $\alpha, \gamma \in L \subset$ $P\left(R^{\vee}\right)$ and $p$ is an LS path. Then $T$ can be unfolded in $X$, i.e., there exists a geodesic triangle $\widetilde{T} \subset X$ such that $g(\widetilde{T})=T$.

Proof. Here is the idea of the proof: We know that billiard triangles in $\Delta$ satisfying the simple chain condition can be unfolded to geodesic triangles in $X$; see Corollary 4.23. Littelmann triangle $T$ is billiard, satisfies the chain condition, but not necessarily the simple chain condition. Our goal is to approximate $T$ by Littelmann polygons $P_{\epsilon}$, $\lim _{\epsilon \rightarrow 0} P_{\epsilon}=T$, which satisfy the simple chain condition. We then unfold each $P_{\epsilon}$ to a geodesic quadrilateral $\widetilde{T}_{\epsilon} \subset X$. Since $X$ is locally compact, there is a convergent 
sequence $\widetilde{T}_{\epsilon_{j}}$ whose limit is a geodesic triangle $\widetilde{T}$ which folds to $T$. Below is the detailed argument.

Consider the geodesic path $\pi_{\beta}=\overline{o b} \in \mathcal{P}^{+}$. Since $p$ is an LS path with the $\Delta$-length $\beta$, according to Property 7 in Section 5.2, there exists a composition $\phi \in \mathcal{F}$ of lowering operators so that

$$
\phi\left(\pi_{\beta}\right)=p .
$$

Let $c \subset \Delta$ denote an alcove which contains the germ of the segment $\overline{o b}$ at $b$. Pick a point $u$ in the interior of $\overline{o b} \cap c$. Then for each $\epsilon>0$ there exists a point $u_{\epsilon} \in \operatorname{int}(c) \cap P\left(R^{\vee}\right) \otimes \mathbb{Q}$ such that

(1) $\left|u-u_{\epsilon}\right|<\epsilon$;

(2) the segments $\overline{o u_{\epsilon}}, \overline{u_{\epsilon} b}$ do not pass through any point of intersection of two or more walls (except for the end-points of these segments).

Observe that $u_{\epsilon}$ is a regular point in $\left(A, W_{\text {aff }}\right)$, i.e., it does not belong to any wall. In other words, the path

$$
\hat{p}_{\epsilon}:=\overline{o u_{\epsilon}} \cup \overline{u_{\epsilon} b} \in \mathcal{P}
$$

is generic. Parameterize $\hat{p}_{\epsilon}$ with the constant speed so that $\hat{p}_{\epsilon}\left(t_{\epsilon}\right)=u_{\epsilon}$. See Figure 9 .

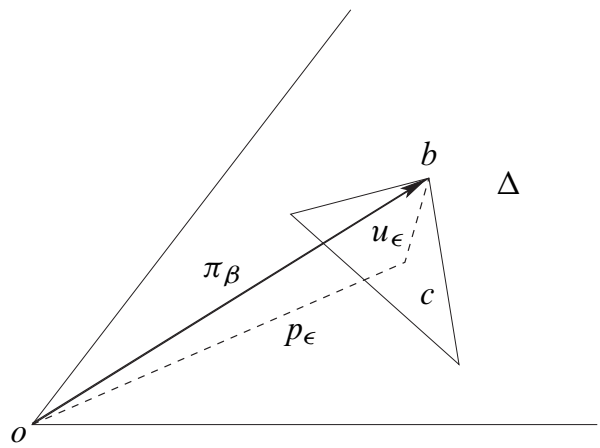

Figure 9. Approximation.

Then the path $\hat{p}_{\epsilon}$ belongs to $\mathcal{P}^{+}$; clearly it is also a generalized LS path: $\hat{p}_{\epsilon} \in \mathscr{L} \mathscr{S}_{2}$. Moreover,

$$
\lim _{\epsilon \rightarrow 0} \hat{p}_{\epsilon}=\pi_{\beta} .
$$

Therefore, according to Property 6 of the root operators (see Section 5.2), the operator $\phi$ is defined on all $\hat{p}_{\epsilon}$ for $\epsilon$ sufficiently small and

$$
\lim _{\epsilon \rightarrow 0} \phi\left(\hat{p}_{\epsilon}\right)=\phi\left(\pi_{\beta}\right)=p .
$$


Set $p_{\epsilon}:=\phi\left(\hat{p}_{\epsilon}\right)$. Since $\hat{p}_{\epsilon}$ was generic, the path $p_{\epsilon}$ is generic as well. By construction, for each sufficiently small $\epsilon$,

$$
p_{\epsilon}(1)=p(1) .
$$

Observe also that the germ of the path $p_{\epsilon}$ at the point $p_{\epsilon}\left(t_{\epsilon}\right)$ is isomorphic (via an element of $W_{\text {aff }}$ ) to the germ of $\hat{p}_{\epsilon}$ at $u_{\epsilon}$ (since $u_{\epsilon}$ is regular). Similarly, $p_{\epsilon}$ is the composition of the path $p_{\epsilon} \mid\left[0, t_{\epsilon}\right]$ with the path that belongs to the $W_{\text {aff }}$-orbit of $\overline{u_{\epsilon} b}$.

For each $\epsilon$ we form a new polygon $P_{\epsilon}$ by replacing the broken side $r(t)=\alpha+p(t)$ (in $T^{\prime}$ ) with the path $\alpha+p_{\epsilon}(t)$. Clearly,

$$
\lim _{\epsilon \rightarrow 0} P_{\epsilon}=T \text {. }
$$

To simplify the notation we now fix $\epsilon>0$ and let $q:=p_{\epsilon}$.

Lemma 6.2. For all sufficiently small $\epsilon$, the polygon $P_{\epsilon}$ is contained in $\Delta$.

Proof. Suppose that $\lambda$ is a simple root which is negative at some point of the path $\alpha+q(t)$.

Since $\lambda$ is nonnegative on the limiting path $\alpha+p$, the minimum of the function $J_{\lambda}(t):=\lambda(q(t)), t \in[0,1]$, converges to zero as $\epsilon \rightarrow 0$. However, as a generalized LS path, $q$ belongs to $\mathcal{P}_{\mathbb{Z}}$ (see Property 5 in Section 5.2). Since $\alpha \in P\left(R^{\vee}\right)$, it follows that the minimum of $J_{\lambda}(t)$ is an integer. Hence it has to be equal to zero for all sufficiently small values of $\epsilon$. Contradiction.

Since each $q$ is a generalized LS path and $P_{\epsilon} \subset \Delta$, the polygon $P_{\epsilon}$ is a Littelmann polygon. Moreover, since $q$ is generic, the polygon $P_{\epsilon}$ satisfies the simple chain condition. Thus

(1) for each $t \in\left[0, t_{\epsilon}\right)$ either $q$ is smooth at $t$ or

$$
\left(q_{-}^{\prime}(t), q_{+}^{\prime}(t)\right)
$$

is a chain of length 1: at $m=q(t)$ the above tangent vectors are related by a single reflection in $W_{m}$, the fixed-point set of this reflection is the unique wall passing through $m$;

(2) the subpath $q\left(\left[t_{\epsilon}, 1\right]\right)$ in $q$ is a geodesic segment and

$$
\delta(\epsilon):=\angle\left(\overrightarrow{u_{\epsilon} \sigma}, \overrightarrow{u_{\epsilon} b}\right)=\pi-\angle\left(q_{-}^{\prime}(t(\epsilon)), q_{+}^{\prime}(t(\epsilon))\right) .
$$

Now we are in a position to apply Corollary 4.23 and unfold $P_{\epsilon}$ in $X$ : For each $\epsilon$ there exists a geodesic quadrilateral $\widetilde{T}_{\epsilon}$ (with one vertex at $o$ ) in $X$ such that

$$
g\left(\widetilde{T}_{\epsilon}\right)=P_{\epsilon} .
$$


Let $\tilde{z}=\tilde{z}_{\epsilon}$ denote the point of $\widetilde{T}_{\epsilon}$ which maps to $z=q\left(t_{\epsilon}\right)$ under the folding map $f$. Since $z$ is a regular point, the point $\tilde{z}$ is regular as well and the angle between the sides of $\widetilde{T}_{\epsilon}$ at $\tilde{z}$ is the same as the angle between the sides of $P_{\epsilon}$ at $z$, i.e., equals $\delta(\epsilon)$.

Since the building $X$ is locally compact, the sequence of quadrilaterals $\widetilde{T}_{\epsilon}$ subconverges to a geodesic quadrilateral $T \subset X$ which is a geodesic triangle since

$$
\lim _{\epsilon \rightarrow 0} \delta(\epsilon)=\pi \text {. }
$$

By continuity of the folding $g: X \rightarrow \Delta$,

$$
g(T)=\lim _{\epsilon \rightarrow 0} P_{\epsilon}=T .
$$

In the above proof we assumed that the polygon $T$ is entirely contained in $\Delta$. This assumption can be weakened. Let $f: X \rightarrow A$ denote the folding Fold $a, A$ into the apartment $A$, where $a$ is an alcove containing $o$. Suppose that $T \subset A$ is as above, so that $\alpha, \gamma \in P\left(R^{\vee}\right), p$ is a billiard path, $r=p+\alpha$. Define two subsets $J, J^{\prime} \subset I=[0,1]$ :

$$
J:=\operatorname{cl}\left(r^{-1}(\operatorname{int}(\Delta))\right), \quad J^{\prime}:=\operatorname{cl}\left(r^{-1}(\operatorname{int}(V \backslash \Delta))\right) .
$$

Clearly, $I=J \cup J^{\prime}$ and the set $J \cap J^{\prime}$ is finite.

We assume that for each $t \in J$ the germ of $p$ at $t$ satisfies the maximal chain condition, and for each $t \in J^{\prime}$ the germ of $p$ at $t$ is geodesic.

Theorem 6.3. Under the above assumptions the polygon $T$ can be unfolded to a geodesic triangle in $X$ via the retraction $f$.

Proof. Recall that unfolding of $T$ is a local problem of behavior of the path $r$ at the break points (Lemma 4.8), which in our case all occur inside $\Delta$.

We first replace $T$ with the polygon $P=\mathbb{P}(T)$, where $\mathbb{P}=\mathbb{P}_{\Delta}$ is the projection of $A$ to the Weyl chamber $\Delta$. Then, analogously to the proof of Proposition 4.19, the new polygon $P$ still satisfies the maximal chain condition. So, according to the previous theorem, the polygon $P$ unfolds in $X$ via the folding map $g=$ Fold $_{\Delta}: X \rightarrow \Delta$. However this means that the unfolding condition (stated in Lemma 4.8) is satisfied at each break point of the polygon $T$ (since the germs of $r$ and of $\mathbb{P}(r)$ are the same). Hence the original polygon $T$ unfolds to a geodesic triangle in $X$ via $f: X \rightarrow A$.

Let $\underline{G}$ be a connected split semisimple algebraic group with the root system $R$ and the cocharacter lattice $L$ of the maximal torus $\underline{T} \subset \underline{G}$. We let $\underline{G}^{\vee}$ denote its Langlands' dual and set

$$
G^{\vee}:=\underline{G}^{\vee}(\mathbb{C}) .
$$

We assume that $\alpha, \beta, \gamma^{*} \in L$ are dominant weights of $G^{\vee}$ such that

$$
\left(V_{\alpha} \otimes V_{\beta} \otimes V_{\gamma^{*}}\right)^{G^{\vee}} \neq 0,
$$


or equivalently,

$$
V_{\gamma} \subset V_{\alpha} \otimes V_{\beta} .
$$

As a corollary of Theorem 6.1 we get a new proof of

Theorem 6.4 (Theorem 9.17 in [KLM3], also proven in [Ha]). Under the above assumptions, in the thick Euclidean building $X$ there exists a geodesic triangle with special vertices and the $\Delta$-side lengths $\alpha, \beta, \gamma^{*}$. In other words,

$$
n_{\alpha, \beta}(\gamma) \neq 0 \Longrightarrow m_{\alpha, \beta}(\gamma) \neq 0 \text {. }
$$

Proof. Since

$$
V_{\gamma} \subset V_{\alpha} \otimes V_{\beta}
$$

according to Littelmann's Theorem 5.21, there exists a Littelmann triangle $T^{\prime} \subset \Delta$, as in Theorem 6.1. Let $p \in \mathcal{P}$ denote the LS path (of the $\Delta$-length $\beta$ ) representing the broken side of $T^{\prime} ; p=\phi\left(\pi_{\beta}\right)$, where $\phi \in \mathcal{F}$ is a composition of lowering operators. Thus, by Theorem 6.1 , there exists a triangle $T=[o, x, y] \subset X$ such that Fold $_{\Delta}(T)=T^{\prime}$. Therefore, by the definition of folding,

$$
d_{\text {ref }}(o, x)=d_{\text {ref }}\left(o, x^{\prime}\right)=\alpha, \quad d_{\text {ref }}(o, y)=d_{\text {ref }}\left(o, y^{\prime}\right)=\gamma .
$$

Since we assumed that $\alpha, \beta \in L \subset P\left(R^{\vee}\right)$ then $x, y$ are special vertices of $x$. Since folding preserves the $\Delta$-length,

$$
d_{\Delta}(x, y)=\operatorname{length}_{\Delta}(p)=\beta .
$$

6.2. Characterization of folded triangles. The goal of this section is to extend the results of the previous one from the case of Littelmann triangles to general broken triangles satisfying the chain condition.

Theorem 6.5. Suppose that $p \in \mathcal{P}$ is a Hecke path, $\alpha=\overrightarrow{o u} \in \Delta$ is such that the path $q:=\alpha+p$ is contained in $\Delta$. Define the billiard triangle $T^{\prime}:=\overline{o u} \cup q \cup \overline{q(1) o}$. Then $T^{\prime}$ can be unfolded in $X$.

Proof. The idea of the proof is that the set of unfoldable billiard paths is closed, thus it suffices to approximate $p$ by unfoldable paths. We first prove the theorem in the case when $o$ does not belong to the image of the path $q$.

According to Lemma 4.8, unfolding of a path is a purely local matter. Therefore the problem reduces to the case when $q$ has only one break point, $x=q\left(t_{1}\right)$. If $p$ were an LS path, we would be done. In general it is not, for instance, because it might fail the maximal chain condition. We resolve this difficulty by passing to a smaller Coxeter complex and a smaller building.

Let $R_{x}$ denote the root subsystem in $R$ which is generated by the roots corresponding to the walls passing through $x$. This root system determines a Euclidean Coxeter 
complex where the stabilizer of the origin is a finite Coxeter group $W_{\mathrm{sph}}^{\prime}$ which is conjugate to the group $W_{x}$ via the translation by the vector $\overrightarrow{o x}$. Let $\Delta_{x}$ denote the positive Weyl chamber of $\left(V, W_{\mathrm{sph}}^{\prime}\right)$ (the unique chamber which contains $\Delta$ ). Let $\xi, \eta$ and $\zeta$ denote the normalizations of the vectors $-p_{-}^{\prime}\left(t_{1}\right), p_{+}^{\prime}\left(t_{1}\right), \overrightarrow{x o}$.

Then, since $p$ satisfies the chain condition, there exists an $\left(S, W_{x}, \Delta_{x}\right)$-chain

$$
\left(v_{0}, \ldots, v_{m}\right), \quad v_{0}=-\xi, v_{m}=\eta, v_{i}=\tau_{i}\left(v_{i-1}\right), 1 \leq i \leq m .
$$

Our first observation is that although this chain may fail to be a maximal chain with respect to the unrestricted Coxeter complex $\left(S, W_{\mathrm{sph}}\right)$, we can assume that it is maximal with respect to the restricted Coxeter complex $\left(S, W_{x}\right)$.

Next, the initial and final points of $q$ may not belong to $P\left(R_{x}^{\vee}\right)$. Recall however that rational points are dense in $S$, see Lemma 2.3; therefore, there exists a sequence of rational points (with respect to $R_{x}$ ) $\xi_{j} \in S$ which converges to $\xi$. Thus, using the same reflections $\tau_{i}$ as before, we obtain a sequence of rational chains $\left(v_{i}^{j}\right), i=0, \ldots, m$, where $v_{0}^{j}=-\xi_{j}, v_{m}^{j}=\eta_{j}$. We set $\zeta_{j}:=\zeta$.

Hence for each $j$ there exists a number $c=c_{j} \in \mathbb{R}_{+}$so that the points

$$
x_{j}=x+c \xi_{j}, \quad y_{j}:=x+c \eta_{j}
$$

belong to $P\left(R^{\vee}\right)$. We define a sequence of paths

$$
q_{j}:=\overline{x_{j} x} \cup \overline{x y_{j}} \in \tilde{\mathcal{P}} .
$$

Our next goal is to choose the sequence $\xi_{j}$ so that the germ of each $q_{j}$ at $x$ is contained in $\Delta$. If $x$ belongs to the interior of $\Delta$ then we do not need any restrictions on the sequence $\xi_{j}$. Assume therefore that $x$ belongs to the boundary of $\Delta$. Let $F$ denote the smallest face of the Coxeter complex $\left(V, W_{\text {sph }}\right)$ which contains the point $x$ and let $H$ denote the intersection of all walls through the origin which contain $x$. It is clear that $F$ is a convex homogeneous polyhedral cone contained in $H$ and $x$ belongs to the interior of $F$ in $H$. If $w \in W_{x}$ is such that $w(-\xi)=\eta$ then $w$ fixes $H$ (and $F$ ) pointwise.

By Lemma 2.3, applied to the root system $R_{x}$, there exists a sequence of unit rational vectors $\xi_{j}$ and positive numbers $\epsilon_{j}$ converging to zero so that points $x \pm \epsilon_{j} \xi_{j}$ belong to $\operatorname{int}_{H}(F)$; therefore the sequence $w\left(x+\epsilon_{j} \xi_{j}\right)$ is also contained in $\operatorname{int}_{H}(F)$.

Using this sequence $\xi_{j}$ we define the paths $q_{j}$; clearly the germ of $q_{j}$ at $x$ is contained in $\operatorname{int}_{H}(F) \subset \Delta \subset \Delta_{x}$.

Remark 6.6. Note that, typically, the sequence $\left(c_{j}\right)$ is unbounded and the paths $q_{j}$ are not contained in $\Delta_{x}$.

We let $p_{j} \in \mathcal{P}$ denote the path $q_{j}-q_{j}(0)$. Then each $p_{j}$ is an LS path with respect to the root system $R_{x}$ : Integrality and the maximal chain condition now hold. Set

$$
\lambda_{j}:=\text { length }_{\Delta_{x}}\left(p_{j}\right) .
$$


Remark 6.7. Observe that

$$
\lim _{j} \bar{\lambda}_{j}=\bar{\lambda} \in \Delta
$$

where $\lambda$ is the $\Delta_{x}$-length of $p$.

Therefore, according to Theorem 6.3 for each $j$ the path $q_{j}$ is unfoldable in a thick Euclidean building $X_{x}$ modeled on the Coxeter complex

$$
\left(A, W_{\text {aff }}^{\prime}\right), \quad \text { where } W_{\text {aff }}^{\prime}=V \ltimes W_{x} .
$$

This means that there exists a geodesic path $\tilde{q}_{j}$ in $X_{x}$, whose $\Delta_{x}$-length is $\lambda_{j}$, and which projects to $q_{j}$ under the folding $X_{x} \rightarrow A$.

Let $z_{j} \in \tilde{q}_{j}$ be the points which correspond to the point $x$ under the folding map

$$
\tilde{q}_{j} \rightarrow q_{j}
$$

So the "broken triangle" $\left[\xi_{j}, \zeta, \eta_{j}\right]$ in $S_{x}$ unfolds in $\Sigma_{z_{j}}\left(X_{x}\right)$ into a triangle $\left[\tilde{\xi}_{j}, \tilde{\zeta}_{j}, \tilde{\eta}_{j}\right]$ such that

$$
\begin{aligned}
d_{\text {ref }}\left(\tilde{\xi}_{j}, \tilde{\zeta}_{j}\right) & =d_{\text {ref }}\left(\xi_{j}, \zeta_{j}\right), \\
d_{\text {ref }}\left(\tilde{\eta}_{j}, \tilde{\zeta}_{j}\right) & =d_{\text {ref }}\left(\eta_{j}, \zeta_{j}\right), \\
d\left(\tilde{\eta}_{j}, \tilde{\zeta}_{j}\right) & =\pi
\end{aligned}
$$

Observe that the metric distance from $o$ to $z_{j}$ is uniformly bounded. Since $X_{x}$ is locally compact, the sequence of buildings $\Sigma_{z_{j}}\left(X_{x}\right)$ subconverges to the link of a vertex $u \in X_{x} \subset X$.

Remark 6.8. The spherical buildings $\Sigma_{z_{j}}\left(X_{x}\right), \Sigma_{u}\left(X_{x}\right)$ have to be modeled on the same spherical Coxeter complex $\left(S, W_{x}\right)$, since the structure group can only increase in the limit and the structure group at $z_{j}$ was already maximal possible, i.e., $W_{x}$.

Accordingly, the triangles $\left[\tilde{\xi}_{j}, \tilde{\zeta}_{j}, \tilde{\eta}_{j}\right]$ subconverge to a triangle $[\tilde{\xi}, \tilde{\zeta}, \tilde{\eta}]$ whose refined side lengths are

$$
d_{\text {ref }}(\xi, \zeta), \quad d_{\text {ref }}(\zeta, \eta), \quad \pi
$$

This shows that the triangle $[\xi, \zeta, \eta]$ can be unfolded in a building which is modeled on $\left(S, W_{x}\right)$. We now apply the locality lemma 4.8 to conclude that the path $q$ can be unfolded in $X$ to a geodesic path. Thus the broken triangle $T^{\prime}$ unfolds to a geodesic triangle as well.

If $o$ belongs to the image of $q$ we argue as follows. The path $q$, as before, has only one break point, which in this case occurs at the origin:

$$
q=\overline{z o} \cup \overline{o y} .
$$


There exists an element $w \in W_{\text {sph }}$ which sends the vector $\eta=-\xi$ to $\xi$, where $\eta$ is the normalization of the vector $\overrightarrow{o y}$. Then consider the geodesic path

$$
\tilde{q}:=\overline{z o} \cup w(\overline{o y}) .
$$

It is clear that $g(\tilde{q})=\mathbb{P}(\tilde{q})=q$.

By combining Theorem 6.5 and Corollary 4.21 we obtain the following

Theorem 6.9 (Characterization of folded triangles). A polygon $P \subset \Delta$ of the form

$$
\overline{o x} \cup(p+\alpha) \cup \overline{y o}, \quad \text { where } \alpha=\overrightarrow{o x}, \gamma=\overrightarrow{o y} \in \Delta,
$$

can be unfolded to a geodesic triangle in $X$ if and only if $p$ is a Hecke path, i.e., a billiard path which satisfies the chain condition.

\section{Proof of the saturation theorem}

We first prove Theorem 1.6 formulated in the Introduction. Part 1 of the theorem was proven in [KLM3], so we prove part 2. Let $X$ be a (thick) Euclidean building of rank $r$ modeled on a discrete Coxeter complex $(A, W)$; the building $X$ is the Bruhat-Tits building associated with the group $G=\underline{G}(\mathbb{K})$. Then the assumption that $m_{\alpha, \beta}(\gamma) \neq 0$ is equivalent to the assumption that there exists a geodesic triangle $T=[\tilde{x}, \tilde{y}, \tilde{z}] \subset X$, where $\tilde{x}, \tilde{y}, \tilde{z}$ are special vertices of $X$ and whose $\Delta$-side lengths are $\alpha, \beta, \gamma^{*} \in \Delta \cap P\left(R^{\vee}\right)$.

Recall that there exists an apartment $\tilde{A} \subset X$ which contains the segment $\overline{\tilde{x}} \tilde{y}$. We let $\tilde{W}$ denote the affine Weyl group operating on $\tilde{A}$. Our first step is to replace the geodesic triangle $T$ with a geodesic polygon

$$
\tilde{P}:=\left[\tilde{z}, \tilde{x}=\tilde{x}_{1}, \ldots, \tilde{x}_{n}, \tilde{x}_{n+1}=\tilde{y}\right]
$$

as follows. We now treat the point $\tilde{x}$ as the origin in the affine space $\tilde{A}$. Let $\tilde{\Delta} \subset \tilde{A}$ be a Weyl chamber in $(\tilde{A}, \tilde{W})$, so that $\tilde{\Delta}$ has its tip at $\tilde{x}$ and $\tilde{x} \tilde{y} \subset \tilde{\Delta}$.

Consider the vectors $\varpi_{1}, \ldots, \varpi_{r} \in \tilde{\Delta}$ which are the fundamental coweights of our root system. Then the vector $\overrightarrow{\tilde{x} \vec{y}}$ is the integer linear combination

$$
\overrightarrow{\tilde{x}} \vec{y}=\sum_{i=1}^{r} n_{i} \varpi_{i}, n_{i} \in \mathbb{N} \cup\{0\} .
$$

Accordingly, we define a path $\tilde{p}$ in $\tilde{\Delta}$ with the initial vertex $\tilde{x}$ and the final vertex $\tilde{y}$ as the concatenation

$$
\tilde{p}=\pi_{\underline{\lambda}}=\pi_{\lambda_{1}} * \cdots * \pi_{\lambda_{r}}=\tilde{p}_{1} \cup \cdots \cup \tilde{p}_{r},
$$


where $\lambda_{i}:=n_{i} \varpi_{i}, \underline{\lambda}=\left(\lambda_{1}, \ldots, \lambda_{r}\right)$. Observe that the path $\tilde{p}$ satisfies the assumptions of Theorem 4.16. Moreover, $\tilde{p}$ is a generalized Hecke path.

Next, let $A \subset X$ be an apartment containing $\overline{\tilde{z}} \tilde{x}, a \subset A$ be an alcove containing $\tilde{z}$; consider the retraction $f=$ Fold $_{a, A}: X \rightarrow A$. This retraction transforms $\widetilde{P}$ to a polygon $\hat{P}:=f(\widetilde{P}) \subset A$ which has geodesic sides $f(\overline{\tilde{z}} \tilde{x}), f(\overline{\tilde{y}} \tilde{z})$. Note that the break points in

$$
\hat{p}:=f(\tilde{p})=\hat{p}_{1} \cup \cdots \cup \hat{p}_{r},
$$

are the images of the vertices $\tilde{x}_{i}$ of $\widetilde{P}$ which are break points $\tilde{p}$, but in addition we possibly have break points within the segments $f\left(\tilde{p}_{i}\right)$. The latter can occur only at the values of $t$ for which the geodesic segments of $\tilde{p}_{i}$ intersect transversally the walls of $(\tilde{A}, \tilde{W})$. Since $\tilde{x}$ is a special vertex and the edges of each $\tilde{p}_{i}$ are parallel to multiples of $\varpi_{i}$, it follows that each segment $\tilde{p}_{i}$ is contained in the 1-skeleton of $X$. Thus the break points of $f\left(\tilde{p}_{i}\right)$ are automatically vertices of $\tilde{A}$. We subdivide the path $\tilde{p}$ so that all break points of $\hat{p}$ are the images of the vertices of $\widetilde{P}$.

Let $k=k_{R}$ be the saturation constant of the root system $R$. Then, according to Lemma 2.8, for each vertex $v \in A$, the point $k v \in A$ is a special vertex of $A$. Therefore, applying a dilation $h \in \operatorname{Dil}(A, W)$ with the conformal factor $k$ to the polygon $\hat{P}$, we obtain a new polygon $k \cdot \hat{P}=h(\hat{P})$, whose vertices are all special vertices of $A$. Thus we can identify the Weyl chamber $\Delta$ with a chamber in $A$ whose tip $o$ is at the vertex $h(\tilde{z})$ and which contains the geodesic segment $h(\overline{\tilde{z}} \tilde{x})$.

Lastly, let $P=\mathbb{P}(k \hat{P})=g(\widetilde{P})$ denote the projection of the polygon $k \hat{P}$ to the Weyl chamber $\Delta$, where $g=$ Fold $_{z, h, \Delta}$. We set $x:=g(\tilde{x}), y:=g(\tilde{y}), x_{i}:=g\left(\tilde{x}_{i}\right)$, $p:=g(\tilde{p})$, etc.

Proposition 7.1. $P=\overline{o x} \cup p \cup \overline{y o}$ is a Littelmann polygon such that

$$
\text { length }_{\Delta}(p)=k \cdot \text { length }_{\Delta}(\tilde{p}) \text {. }
$$

Proof. We have to show that the path $p$ satisfies the chain condition with maximal chains at each vertex.

The chain condition at each vertex of the path $p$ follows immediately from Theorem 4.16.

The maximality condition is immediate for the break points which occur at the special vertices of $A$, in particular, for all break points which are images of the break points of $\hat{p}$. The remaining break points are the ones which occur at the points $\mathbb{P}\left(\hat{x}_{i}\right)$, where $\hat{x}_{i}$ are smooth points of $k \hat{p}$ at which this path transversally intersects the walls of $A$ passing through $o$. However at these points the maximality condition follows from Proposition 4.19.

The second assertion of the proposition was proven in Lemma 4.5.

Remark 7.2. Observe that in the case when $\beta$ is the sum of minuscule fundamental coweights, the multiplication by $k$ in the above proof is unnecessary since all the 
vertices of the polygon $\hat{P}$ (and hence $P=\mathbb{P}(\hat{P})$ ) are already special. Thus, in this case, the polygon

$$
P=\operatorname{Fold}_{\Delta}(\widetilde{P})
$$

is a Littelmann polygon.

The above proposition shows that the polygon $P$ is a Littelmann polygon in $\Delta$, which has two geodesic sides having the $\Delta$-lengths $k \alpha, k \gamma^{*}$ and the concatenation of the remaining sides equal to a generalized LS path of the $\Delta$-length $k \beta$. Therefore, according to Littelmann's theorem (see Theorem 5.21),

$$
V_{k \gamma} \subset V_{k \alpha} \otimes V_{k \beta} \text {. }
$$

This concludes the proof of Theorem 1.6.

Corollary 7.3. Suppose that $\alpha, \beta, \gamma \in L \subset P\left(R^{\vee}\right)$ are dominant weights for the complex semisimple Lie group $\underline{G}^{\vee}(\mathbb{C})$, such that $\alpha+\beta+\gamma \in Q\left(R^{\vee}\right)$ and that there exists $N \in \mathbb{N}$ so that

$$
V_{N \gamma} \subset V_{N \alpha} \otimes V_{N \beta} .
$$

Then for the saturation constant $k=k_{R}^{2}$ we have

$$
V_{k \gamma} \subset V_{k \alpha} \otimes V_{k \beta} .
$$

Proof. Let $X$ be the Euclidean (Bruhat-Tits) building associated to the group $\underline{G}(\mathbb{K})$. Then the assumption that

$$
V_{N \gamma} \subset V_{N \alpha} \otimes V_{N \beta}
$$

implies that $\left(N \alpha, N \beta, N \gamma^{*}\right)$ belongs to $D_{3}(X)$ (see [KLM3], Theorem 9.17, or [KLM3], Theorem 10.3, or Theorem 6.4). Since $D_{3}(X)$ is a homogeneous cone and $N>0,\left(\alpha, \beta, \gamma^{*}\right) \in D_{3}(X)$ as well. Moreover, according to Theorem 2.17, since $\alpha, \beta, \gamma \in P\left(R^{\vee}\right)$ and $\alpha+\beta+\gamma \in Q\left(R^{\vee}\right)$, there exists a triangle $T \subset X$ with the $\Delta$-side lengths $\alpha, \beta, \gamma^{*}$, whose vertices are also vertices of $X$. Now the assertion follows from Part 3 of theorem 1.6.

Using Remark 7.2 we also obtain:

Theorem 7.4. Let $X$ be a building as above. Suppose that $T=[x, y, z]$ is a geodesic triangle in $X$ with the $\Delta$-side lengths $\left(\alpha, \beta, \gamma^{*}\right)$, which are dominant weights of $G^{\vee}$ and so that one (equivalently, all) vertices of $T$ are special and at least one of the weights $\alpha, \beta, \gamma$ is the sum of minuscule weights. Then

$$
V_{\gamma} \subset V_{\alpha} \otimes V_{\beta} .
$$

This theorem was originally proven by Tom Haines in the case when all the weights $\alpha, \beta, \gamma$ are sums of minuscules. 
Conjecture 7.5 (T. Haines). Suppose that $\alpha, \beta, \gamma$ are sums of minuscule weights. Then, in the above theorem, the assumption that one vertex of $T$ is special can be replaced by $\alpha+\beta+\gamma \in Q\left(R^{\vee}\right)$.

Note that (among irreducible root systems) the root systems $G_{2}, F_{4}, E_{8}$ have no minuscule weights, $B_{n}, C_{n}, E_{7}$ have exactly one minuscule weight and the root systems $A_{n}, D_{n}, E_{6}$ have more than one minuscule weights. For the root system $A_{n}$ Haines conjecture follows from the saturation theorem. For $D_{n}$ and $E_{6}$ it would follow from the affirmative answer to Question 1.3.

Proposition 7.6. Suppose that the root system $R$ has exactly one minuscule coweight $\lambda$. Then the above conjecture holds for $R$.

Proof. Let $(A, W)$ denote the Euclidean Coxeter complex corresponding to the root system $R$ and let $X$ be a thick Euclidean building modeled on $(A, W)$. Given $\left(\alpha, \beta, \gamma^{*}\right) \in D_{3}(X)$ such that $\alpha, \beta, \gamma \in P\left(R^{\vee}\right), \alpha+\beta+\gamma \in Q\left(R^{\vee}\right)$ we have to construct a geodesic triangle $T=[o, x, y] \subset X$ with special vertices and the $\Delta$-side lengths $\left(\alpha, \beta, \gamma^{*}\right)$. Clearly, it suffices to treat the case when the root system $R$ is irreducible and spans $V$. Therefore $R$ has type $B_{n}, C_{n}$, or $E_{7}$. In particular, the index of connection $i$ of $R$ equals 2 and $-1 \in W_{\text {sph }}$. In particular, $\gamma=\gamma^{*}$. Let $\lambda$ denote the unique minuscule coweight of $R$ and let $\Lambda$ denote the span in $\lambda$ in $V$.

Observe that $\lambda$ does not belong to the coroot lattice $Q\left(R^{\vee}\right)$ and thus, since $i=2$,

$$
\mathbb{N} \cdot \lambda \cap Q\left(R^{\vee}\right)=2 \mathbb{N} \cdot \lambda .
$$

Suppose now that $\alpha=a \lambda, \beta=b \lambda, \gamma=b \lambda$, where $a, b, c \in \mathbb{N} \cup\{0\}$ and

$$
(\alpha, \beta, \gamma) \in D_{3}(X), \quad \alpha+\beta+\gamma \in Q\left(R^{\vee}\right) .
$$

Thus $a+b+c$ is an even number and the triple $(a, b, c)$ satisfies the ordinary metric triangle inequalities.

Let $\left(A^{\prime}, W^{\prime}\right)=(\mathbb{R}, 2 \mathbb{Z} \ltimes \mathbb{Z} / 2)$ denote the rank 1 Coxeter complex; its vertex set equals $\mathbb{Z}$. The positive Weyl chamber in $\left(A^{\prime}, W^{\prime}\right)$ is $\mathbb{R}_{+}$and we can identify $\Delta^{\prime}$-distances with the usual metric distances. Let $X^{\prime}$ denote a thick building which is modeled on $\left(A^{\prime}, W^{\prime}\right)$ (i.e., a simplicial tree with edges of unit length and thickness $\geq 3$ ). Then the above properties of $a, b, c$ imply that $X^{\prime}$ contains a triangle $T^{\prime}=\left[o^{\prime}, x^{\prime}, y^{\prime}\right]$ with the metric side lengths $a, b, c$. If this triangle is contained in a single apartment $A^{\prime} \subset X^{\prime}$, we send $T^{\prime}$ to a geodesic triangle $T \subset X$ via the isometry $A^{\prime} \rightarrow \Lambda \rightarrow A \rightarrow X$. If not, we obtain a folded (Hecke) triangle $P=f^{\prime}\left(T^{\prime}\right)=$ $\left[o^{\prime}, x^{\prime}, u^{\prime}, f^{\prime}\left(y^{\prime}\right)\right] \subset \Delta^{\prime}$. Note that the unit tangent directions $\xi^{\prime}, \eta^{\prime} \in S_{u^{\prime}}\left(A^{\prime}\right)$ to the segments $u^{\prime} x^{\prime}, u^{\prime} f\left(y^{\prime}\right)$ are antipodal. Now embed the apartment $A^{\prime}$ into $A \subset X$ via the isometry $\iota$ that sends $A^{\prime}$ to $\Lambda, o^{\prime}$ to $o, 1$ to $\lambda$ (the latter is a special vertex). Then the point $u:=\iota\left(u^{\prime}\right)$ is also a special vertex in $A$. We claim that the resulting broken 
triangle $[o, x, u, y] \subset A$ is a Hecke triangle in $A$. Indeed, the directions $\xi, \eta$ at $\Sigma_{u}$ which are images of $\xi^{\prime}, \eta^{\prime}$ under $\iota$ are antipodal and $\eta \in \Delta$. Therefore, since $u$ is a special vertex and $-1 \in W_{\text {sph }}$, according to Lemma 3.12, $\xi \geq \eta$. Thus $\overline{x u} \cup \overline{u y}$ is a Hecke path. It follows that the broken triangle $[o, x, u, y]$ is a Hecke triangle and hence it unfolds to a geodesic triangle $T$ in $X$. The triangle $T$ has special vertices and $\Delta$-side lengths $(\alpha, \beta, \gamma)$.

Below is an alternative to the above argument. Let $\Sigma \subset \Delta^{3}$ denote the collection of triples of dominant weights $\tau, \eta, \mu$ such that

$$
\left(V_{\tau} \otimes V_{\eta} \otimes V_{\mu}\right)^{G^{\vee}} \neq 0,
$$

where $G^{\vee}$ is assumed to be simply-connected. This set is an additive semigroup; see, for instance, [KLM3], Appendix. It suffices to prove that $(\alpha, \beta, \gamma) \in \Sigma$.

Set $t:=\frac{1}{2}(a+b-c)$. (The number $t$ is the metric length of the "leg" of the geodesic triangle $T^{\prime} \subset X^{\prime}$ in the above argument, the leg which contains the vertex $x^{\prime}$.) Since $a+b+c$ is even, the number $t$ is an integer. Set

$$
a_{1}:=a-t, \quad b_{1}:=b-t, \quad c_{1}:=c, \quad \alpha_{1}:=a_{1} \lambda, \quad \beta_{1}:=b_{1} \beta, \quad \gamma_{1}:=c_{1} \gamma .
$$

Then $c_{1}=a_{1}+b_{1}$ and the metric triangle inequalities for $a, b, c$ imply that $t \geq 0$, $a_{1} \geq 0, b_{1} \geq 0$. Thus $\alpha_{1}, \beta_{1}, \gamma_{1}$ are still dominant weights of $G^{\vee}$ and they satisfy

$$
\gamma_{1}=\alpha_{1}+\beta_{1} .
$$

Then, since $-1 \in W_{\text {sph }}$ and $\gamma_{1}=\gamma_{1}^{*}$, we have $\left(\alpha_{1}, \beta_{1}, \gamma_{1}\right) \in \Sigma$. Moreover, $(t \lambda, t \lambda, 0)$ also clearly belongs to $\Sigma$ and we have

$$
(\alpha, \beta, \gamma)=\left(\alpha_{1}, \beta_{1}, \gamma_{1}\right)+(t \lambda, t \lambda, 0)=(\alpha, \beta, \gamma) .
$$

Example 7.7. There exists a Hecke path $p \in \mathcal{P}$ such that $p(1) \in P\left(R^{\vee}\right)$; however for the saturation constant $k=k_{R}$, the path $k \cdot p$ is not an LS path.

Proof. Our example is for the root system $A_{2}$, in which case $k=1$. We will give an example of a Hecke path $p \in \mathcal{P}$ such that $p(1) \in P\left(R^{\vee}\right)$, but $p$ does not belong to $\mathcal{P}_{\mathbb{Z}}$. Since, according to Theorem 5.5, each LS path belong to $\mathcal{P}_{\mathbb{Z}}$, this proves that $p$ is not an LS path.

The Hecke path $p$ in question has $\Delta$-length $\varpi_{1}+\varpi_{2}$, where $\varpi_{1}, \varpi_{2}$ are the fundamental coweights; the break point of $p$ occurs at the point $-\left(\varpi_{1}+\varpi_{2}\right) / 2$ where the path $p$ backtracks back to the origin. Thus, for the simple roots $\alpha$ and $\beta$, the minimum of the functions $\alpha(p(t)), \beta(p(t))$ equals $-1 / 2$. See Figure 10. 


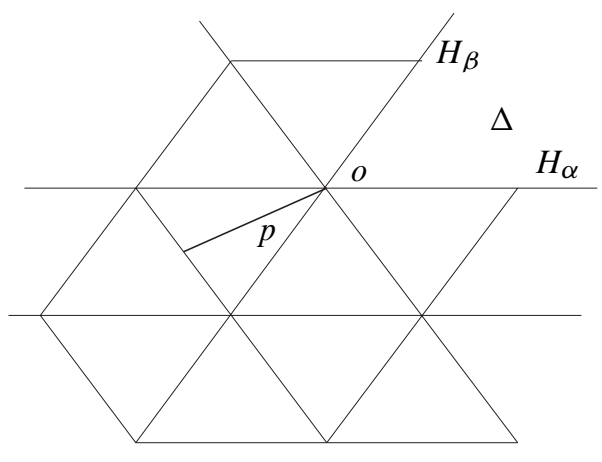

Figure 10. A Hecke path which does not satisfy the integrality condition.

\section{References}

[Ba] W. Ballmann, Lectures on spaces of nonpositive curvature. DMV Seminar 25, Birkhäuser Verlag, Basel 1995. Zbl 0834.53003 MR 1377265

[BK] P. Belkale and S. Kumar, Eigencone, saturation and Horn problems for symplectic and odd orthogonal groups. Preprint 2007. arXiv:0708.0398

[BS] A. Berenstein and R. Sjamaar, Coadjoint orbits, moment polytopes, and the HilbertMumford criterion. J. Amer. Math. Soc. 13 (2000), 433-466. Zbl 0979.53092 MR 1750957

[B] A. Borel, Linear algebraic groups. 2nd ed., Graduate Texts in Math. 126, SpringerVerlag, New York 1991. Zbl 0726.20030 MR 1102012

[Bo] N. Bourbaki, Lie groups and Lie algebras. Chapters 4-6. Springer-Verlag, Berlin 2002. Zbl 0983.17001 MR 1890629

[Br] K. S. Brown, Buildings. Springer Monogr. Math., Springer-Verlag, New York 1998. Zbl 0922.20034 MR 1644630

[BT] F. Bruhat and J. Tits, Groupes réductifs sur un corps local. Inst. Hautes Études Sci. Publ. Math. 41 (1972), 5-251. Zbl 0254.14017 MR 0327923

[D] M. Demazure, Schémas en groupes réductifs. Bull. Soc. Math. France 93 (1965), 369-413. Zbl 0163.27402 MR 0197467

[DW] H. Derksen and J. Weyman, Semi-invariants of quivers and saturation for LittlewoodRichardson coefficients. J. Amer. Math. Soc. 13 (2000), 467-479. Zbl 0993.16011 MR 1758750

[G] P. Garrett, Buildings and classical groups. Chapman \& Hall, London 1997. Zbl 0933.20019 MR 1449872

[GL] S. Gaussent and P. Littelmann, LS galleries, the path model, and MV cycles. Duke Math. J. 127 (2005), 35-88. Zbl 1078.22007 MR 2126496 
[Gro] B. H. Gross, On the Satake isomorphism. In Galois representations in arithmetic algebraic geometry (Durham, 1996), London Math. Soc. Lecture Note Ser. 254, Cambridge University Press, Cambridge 1998, 223-237. Zbl 0996.11038 MR 1696481

[Ha] T. J. Haines, Structure constants for Hecke and representation rings. Internat. Math. Res. Notices 2003 (2003), 2103-2119. Zbl 1071.22020 MR 1995683

[KKM] M. Kapovich, S. Kumar and J. J. Millson, Saturation and irredundancy for Spin(8). Pure Appl. Math. Quart., to appear.

[KLM1] M. Kapovich, B. Leeb, and J. J. Millson, Convex functions on symmetric spaces, side lengths of polygons and the stability inequalities for weighted configurations at infinity. J. Differential Geometry, to appear. arXiv:math/0311486v3

[KLM2] M. Kapovich, B. Leeb and J. J. Millson, Polygons in buildings and their refined side lengths. Preprint 2005. arXiv:math/0406305v2

[KLM3] M. Kapovich, B. Leeb and J. J. Millson, The generalized triangle inequalities in symmetric spaces and buildings with applications to algebra. Mem. Amer. Math. Soc. 192896 (2008) Zbl 05256606 MR 2369545

[KL] B. Kleiner and B. Leeb, Rigidity of quasi-isometries for symmetric spaces and Euclidean buildings. Inst. Hautes Études Sci. Publ. Math. 86 (1997), 115-197. Zbl 0910.53035 MR 1608566

[KT] A. Knutson and T. Tao, The honeycomb model of $G L_{n}(\mathbb{C})$ tensor products I: Proof of the saturation conjecture. J. Amer. Math. Soc. 12 (1999), 1055-1090. Zbl 0944.05097 MR 1671451

[L1] P. Littelmann, A Littlewood-Richardson rule for symmetrizable Kac-Moody algebras. Invent. Math. 116 (1994), 329-346. Zbl 0805.17019 MR 1253196

[L2] P. Littelmann, Paths and root operators in representation theory. Ann. of Math. (2) 142 (1995), 499-525. Zbl 0858.17023 MR 1356780

[L3] P. Littelmann, Characters of representations and paths in $\mathfrak{H}_{\mathbb{R}}^{*}$. In Representation theory and automorphic forms (Edinburgh, 1996), Proc. Sympos. Pure Math. 61, Amer. Math. Soc., Providence, RI, 1997, 29-49. Zbl 0892.17010 MR 1476490

[Ron] M. Ronan, Lectures on buildings. Perspect. Math. 7, Academic Press, Boston 1989. Zbl 0694.51001 MR 1005533

[Rou] G. Rousseau, Euclidean buildings. Lecture notes from Summer School 2004: Nonpositively curved geometries, discrete groups and rigidities, Institut Fourier, Grenoble 2004. http://hal.archives-ouvertes.fr/hal-00094363

[Sat] I. Satake, Theory of spherical functions on reductive algebraic groups over padic fields. Inst. Hautes Études Sci. Publ. Math. 18 (1963), 5-69. Zbl 0122.28501 MR 0195863

[Sc] C. Schwer, Galleries, Hall-Littlewood polynomials, and structure constants of the spherical Hecke algebra. Internat. Math. Res. Notices 2006 (2006), Article ID 75395, 1-31. Zbl 1121.05121 MR 2264725

Received November 25, 2006; revised December 15, 2007 
M. Kapovich, Department of Mathematics, University of California, Davis, CA 95616, U.S.A.

E-mail: kapovich@math.ucdavis.edu

J. J. Millson, Department of Mathematics, University of Maryland, College Park, MD 20742, U.S.A.

E-mail: jjm@math.umd.edu 